OAK RIDGE NATIONAL LABORATORY

\section{MAGTUN MARUETRA}

\section{BERYLLIUM VERIFICATION SURVEY at the
PEEK STREET SITE
SCHENECTADY, NEW YORK at the
PEEK STREET SITE
SCHENECTADY, NEW YORK at the
PEEK STREET SITE
SCHENECTADY, NEW YORK (SY001V) \\ RESULTS OF THE RADIOLOGICAL and}
R. D. Foley
C. A. Johnson
R. F. Carrier
J. F. Allred

\section{MANAGED BY}

MARTIN MARIETTA ENERGY SYSTEMS, INC.

FOR THE UNITED STATES

DEPARTMENT OF ENERGY

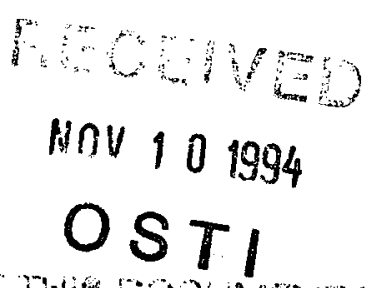

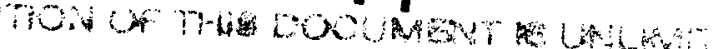


This report has been reproduced directly from the best available copy.

Available to DOE and DOE contractors from the Office of Scientific and Technical Information, P.O. Box 62, Oak Ridge, TN 37831; prices available from (615) 576-8401, FTS 626-8401.

Available to the public from the National Technical Information Service, U.S. Department of Commerce, 5285 Port Royal Rd., Springfield, VA 22161.

This report was prepared as an account of work sponsored by an agency of the United States Government. Neither the United States Government nor any agency thereof, nor any of their employees, makes any warranty, express or implied, or assumes any legal liability or responsibility for the accuracy, completeness, or usefulness of any information, apparatus, product, or process disclosed, or represents that its use would not infringe privately owned rights. Reference herein to any specific commercial product, process, or service by trade name, trademark, manufacturer, or otherwise, does not necessarily constitute or imply its endorsement, recommendation, or favoring by the United States Government or any agency thereof. The views and opinions of authors expressed herein do not necessarily state or reflect those of the United States Government or any agency thereot. 


\section{DISCLAIMER}

This report was prepared as an account of work sponsored by an agency of the United States Government. Neither the United States Government nor any agency Thereof, nor any of their employees, makes any warranty, express or implied, or assumes any legal liability or responsibility for the accuracy, completeness, or usefulness of any information, apparatus, product, or process disclosed, or represents that its use would not infringe privately owned rights. Reference herein to any specific commercial product, process, or service by trade name, trademark, manufacturer, or otherwise does not necessarily constitute or imply its endorsement, recommendation, or favoring by the United States Government or any agency thereof. The views and opinions of authors expressed herein do not necessarily state or reflect those of the United States Government or any agency thereof. 


\section{DISCLAIMER}

Portions of this document may be illegible in electronic image products. Images are produced from the best available original document. 
HEALTH SCIENCES RESEARCH DIVISION

Waste Management Research and Development Programs

(Activity No. 613062401 NOG)

\title{
Results of the Radiological and Beryllium Verification Survey at the Peek Street Site, Schenectady, New York (SY001V)
}

R. D. Foley, C. A. Johnson, R. F. Carrier, J. F. Allred

Date issued -October 1994

\author{
Investigation Team \\ (Site code: SY001V)
}

R.D. Foley - Measurement Applications and Development Manager

W. D. Cottrell - FUSRAP Project Director

This document is

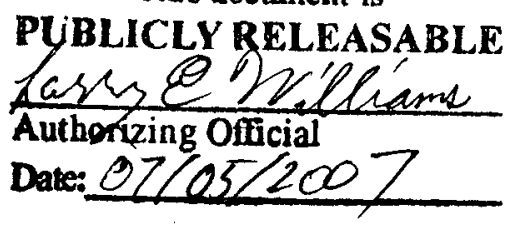

Survey Team Members
J. F. Allred
V. P. Patania
A. C. Butler*
D. E. Rice
R. L. Coleman
D. A. Rose
R. C. Gosslee
K. R. Kleinhans
P. F. Tiner
J. Wade
R. A Mathis
W. Winton

*Midwest Technical, Inc.

Work performed by the

Measurement Applications and Development Group

Prepared by the

OAK RIDGE NATIONAL LABORATORY

Oak Ridge, Tennessee 37831-6285

managed by

MARTIN MARIETTA ENERGY SYSTEMS, INC.

for the

U.S. DEPARTMENT OF ENERGY

under contract DE-AC05-84OR21400 
* $\div \quad \because$

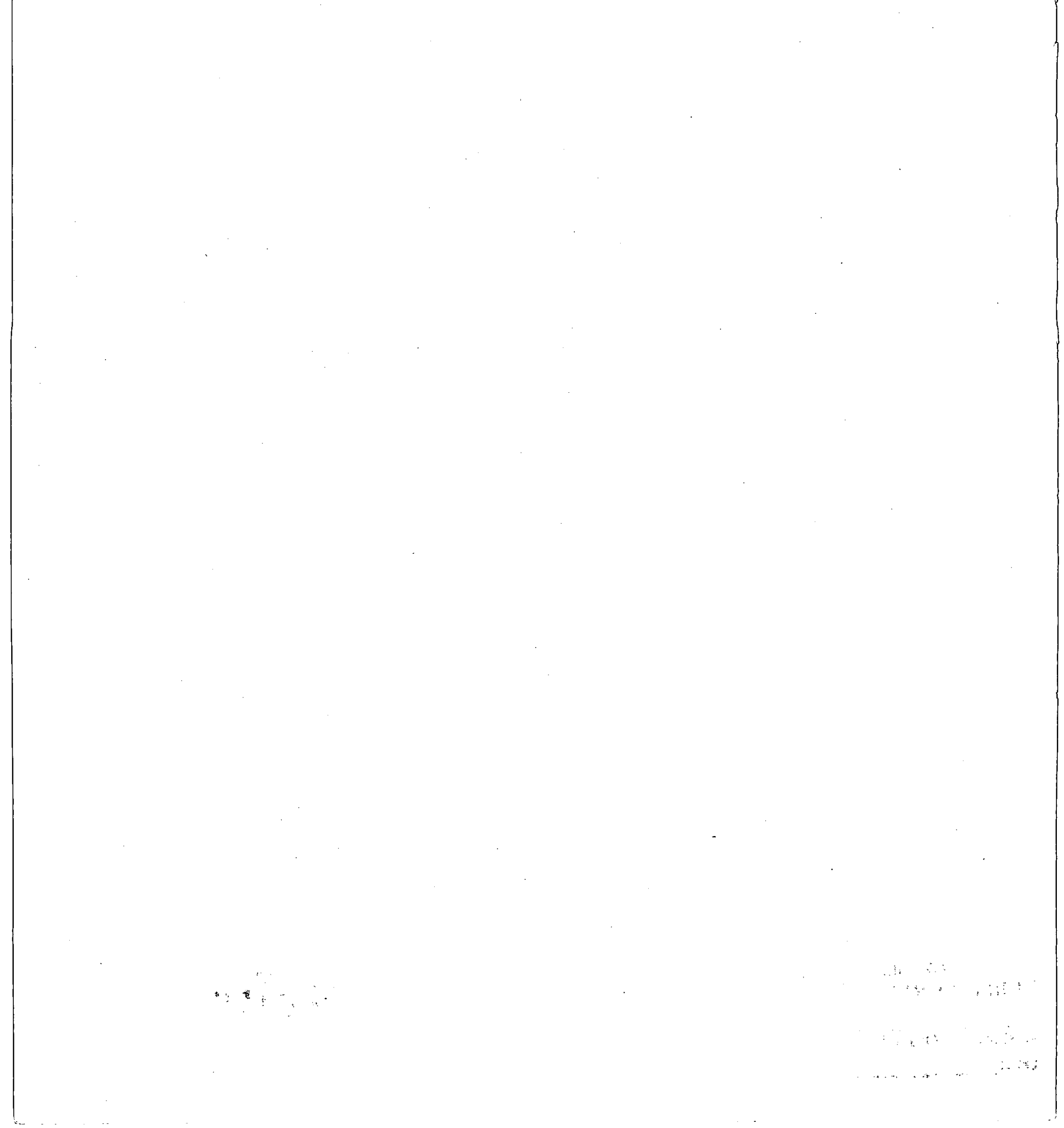




\section{CONTENTS}

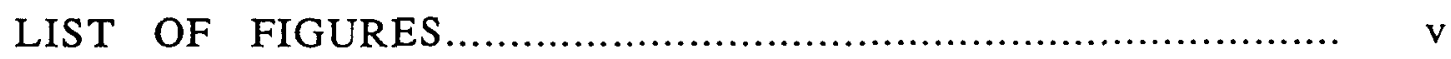

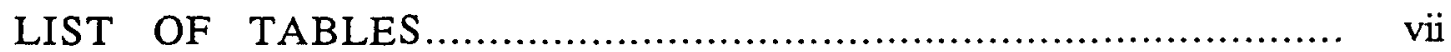

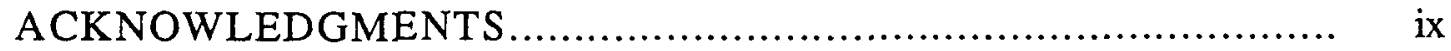

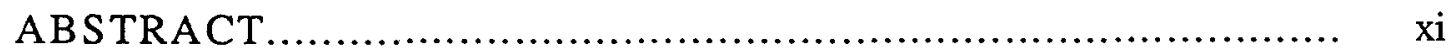

INTRODUCTION................................................... 1

VERIFICATION PROCEDURES.......................................... 2

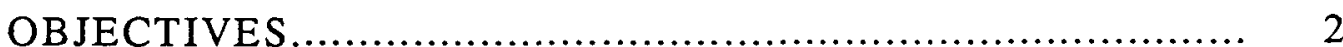

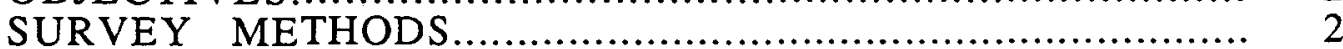

Radiological Survey.......................................... 3

Beryllium Survey............................................. 3

VERIFICATION SURVEY AND ANALYSIS.............................. 4

RADIOLOGICAL SURVEY RESULTS ................................ 4

BERYLLIUM SURVEY RESULTS .................................... 5

OVERCHECKS BY NEW YORK STATE …................................ 5

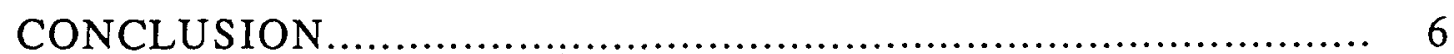

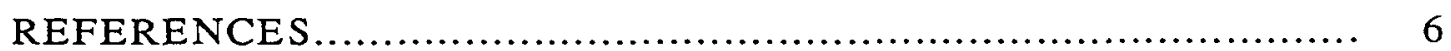




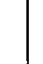

\{

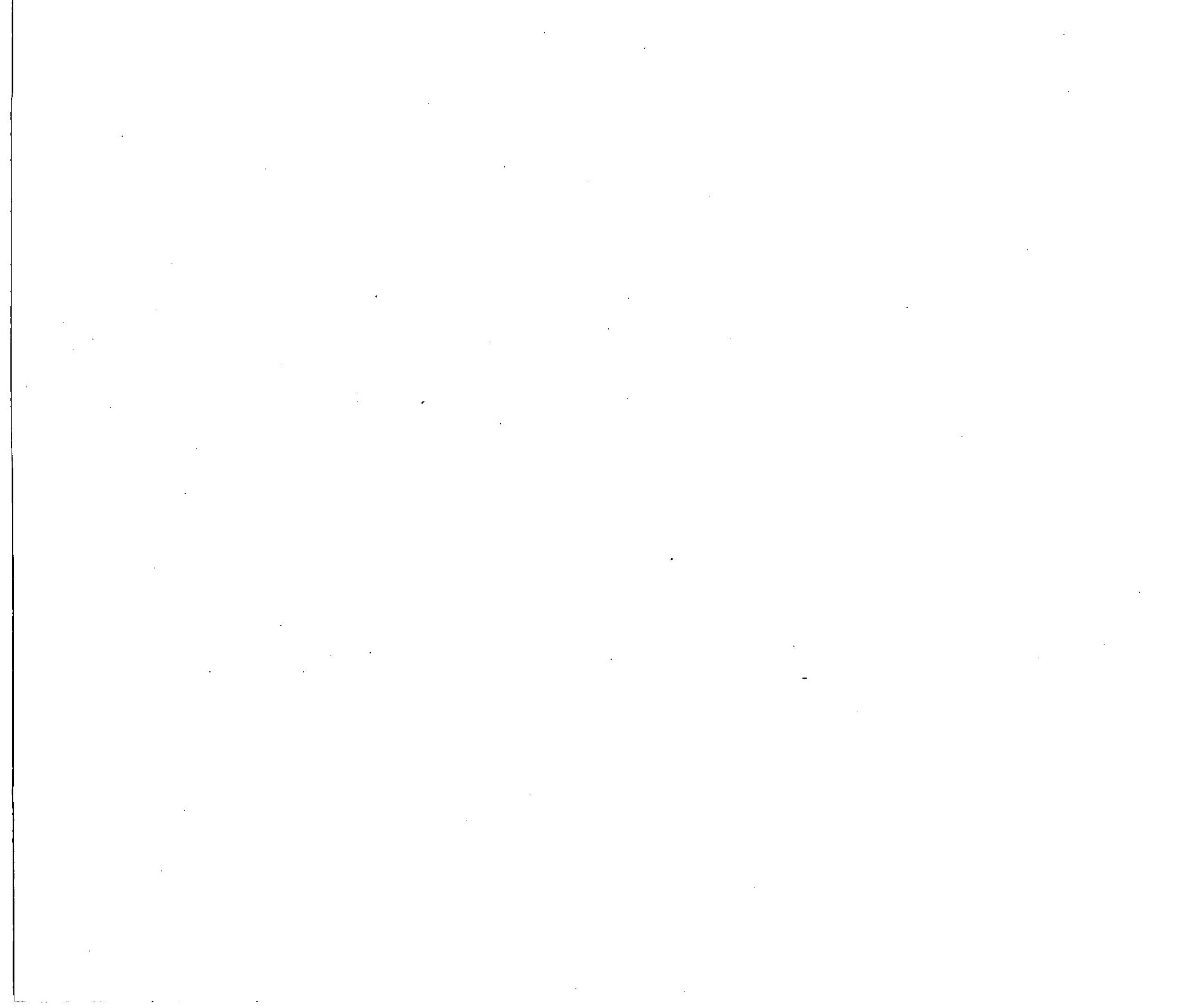




\section{LIST OF FIGURES}

1 Diagram showing the layout of the factory building on the industrial property at 425 Peek Street, Schenectady, New York

2 Diagram showing gamma measurements and soil sampling locations in the gridded area on the east side of the factory building at 425 Peek St., Schenectady, New York.

3 Diagram showing locations of samples and smears relative to the drains and sanitary waste lines inside the factory building at the Peek Street site.

$4 \quad$ Diagram showing the smear and sample locations taken from the floor and various horizontal surfaces inside the factory building.

5 Close up diagram of room MS-10 and adjacent boiler room showing locations of samples and smears taken from the floor and miscellaneous horizontal surfaces.

6 Diagram showing the locations of samples and smears taken from overhead surfaces in the factory building at the Peek Street site

7 Diagram showing locations of smears and samples taken on window ledges, tops of walls, and other horizontal surfaces inside the factory building on the Peek Street site

8 Diagram of the factory building showing general locations of radiological smears taken throughout the building that measured less than minimum detectable activity (<MDA) for transferable alpha and beta-gamma levels. 


\section{LIST OF TABLES}

1 Applicable guidelines for the Peek Street site........................................ 15

2 Background radiation levels and concentrations of selected radionuclides in soil samples taken in the Schenectady and Glenville, New York area........... 16

3 Concentrations of radionuclides in soil samples collected for the independent verification of the property at 425 Peek Street, Schenectady, New York............

4 Alphanumeric listing of samples, locations, and beryllium results for the independent verification survey of 425 Peek Street.................................... 18

5 Additional notations for selected indoor samples collected for the independent verification survey of 425 Peek Street. 


\section{ACKNOWLEDGMENTS}

Research for this project was sponsored by the Office of Naval Reactors, U. S. Department of Energy, under contract DE-AC05-OR21400 with Martin Marietta Energy Systems, Inc. The authors wish to acknowledge the contributions of W. D. Cottrell, D. A. Roberts, D. A. Rose, P. A. Brimer, J. M. Lovegrove and T. R. Stewart of the Measurement Applications and Development Group for participation in the sample preparation and analyses, editing, graphics, and reporting of data for this survey. The surveying assistance of the staff on the survey team is also gratefully acknowledged. The operational and technical assistance of M. J. McGeoch and G. A. Racine of the Knolls Atomic Power Laboratory is gratefully acknowledged. 


\begin{abstract}
At the request of the U.S. Department of Energy (DOE), a team from Oak Ridge National Laboratory conducted an independent verification radiological and non-radioactive beryllium survey at the Peek Street site, located at 425 Peek Street, Schenectady, New York. The purpose of the survey, conducted during 1993 and continuing through January 1994, was to confirm the success of the remedial actions performed to remove any beryllium concentrations or radioactive materials in excess of the identified guidelines. The verification survey included surface gamma scans and gamma readings at one meter indoors and outdoors, alpha and beta scans inside the structure, and the collection of soil, dust and debris samples and smears for radionuclide and beryllium analyses.

Results of the survey demonstrated that all radiological and beryllium measurements on the property were within applicable DOE guidelines. Based on all data collected, the industrial property at 425 Peek Street and the adjacent state-owned bike path in Schenectady, New York, conforms to all applicable radiological and non-radioactive beryllium guidelines established for this site by DOE and approved by the State of New York.
\end{abstract}




\section{RESULTS OF THE RADIOLOGICAL and BERYLLIUM VERIFICATION SURVEY at the PEEK STREET SITE, SCHENECTADY, NEW YORK*}

\section{INTRODUCTION}

Radioactive materials were used in a variety of operations conducted at 425 Peek Street, Schenectady, New York, an industrial facility operated by the General Electric Company for the Atomic Energy Commission (AEC) between 1947 and 1955. Nonradioactive beryllium metal was also machined on the site for breeder reactor application. The 4.5-acre site was decommissioned and released in October 1955.

At the request of the Office of Naval Reactors through the Office of Remedial Action and Waste Technology, a site visit and preliminary scoping survey of the Peek Street facility was conducted by Oak Ridge National (ORNL) in July 1988 and included the participation of representatives from New York State agencies. 1 The major portion of the State-owned bike path received a preliminary survey in November 1988. A comprehensive survey was performed between August and November 1989, with additional sampling in 1990 to definitively characterize current radiological conditions at the site. ${ }^{2}$

The results of that survey indicated the existence of small isolated areas that exceeded DOE radiological guidelines. Samples were also analyzed for elemental beryllium since that material had formerly been used at the site. In conjunction with the planned remediation at the facility, a supplementary characterization survey was performed to further define the areas containing beryllium in excess of the identified guidelines. Additional radiological characterization was also performed in areas that were largely inaccessible prior to the remediation efforts. This supplementary survey was performed by ORNL during the period March through December 1993.3

Site-specific guidelines of $2 \mu \mathrm{g} / \mathrm{ft}^{2}$ for indoor surfaces and $13 \mu \mathrm{g} / \mathrm{g}$ for interior drains and outdoor soil concentrations of beryllium were developed for purposes of the remedial action and verification of the property at Peek Street. Table 1 lists all guidelines derived for this site by the DOE and approved by the State of New York. Not specifically listed in the table is the guideline for beta-gamma surface contamination that was used here where alpha activity levels were masked by structural surface treatments such as paint. That is, the guideline for total indoor alpha surface contamination resulting from $238 \mathrm{U}$ residuals $\left(5,000 \mathrm{dpm} / 100 \mathrm{~cm}^{2}\right.$, Table 1$)$ was also used for directly measured beta-gamma activity levels. In addition, the established guidelines listed in Table 1 for beta-gamma dose rates were applied.

\footnotetext{
${ }^{*}$ The survey was performed by members of the Measurement Applications and Development Group of the Health Sciences Research Division of Oak Ridge National Laboratory under DOE contract DE-AC05840 R21400.
} 
Remedial efforts were instituted by management at the Knolls Atomic Power Laboratory (KAPL), the project Remedial Action Contractor, wherever results of the previous surveys indicated the presence of elevated radiation or non-radioactive, elemental beryllium measurements. Their task was to ensure that radiation levels and radionuclide concentrations met DOE guidelines, and that all beryllium in excess of the established guidelines had been removed. Some of the remedial efforts were undertaken by Argonne National Laboratory (e.g., initial radiological remediation in the vault, the wall and floor around column $26 \mathrm{E}$, and excavation of the outside area east of the center section of the factory building). Additionally, KAPL excavated areas of the concrete floors to gain access to drain headers for further measurements, and removed some drainlines that had exceeded criteria. Drains were either permanently removed or replaced with new pipes, and the area restored. Allwash of Syracuse, Inc., remediation subcontractor, performed the beryllium removal. All remedial actions were completed by December 1993.4

It is DOE policy to assign an Independent Verification Contractor to ensure the effectiveness of such remedial actions. The Measurement Applications and Development Group of Oak Ridge National Laboratory (ORNL) was given responsibility for this task at 425 Peek Street. Because of an overlap between the supplementary characterization survey and the initiation of remedial action at the facility, some data relevant to the characterization were collected during the independent verification survey. This report describes the methods and results of the verification survey that was conducted during 1993 and continued until mid-January 1994. Figure 1 is a diagram of the layout of the factory building on the industrial property at 425 Peek Street.

\section{VERIFICATION PROCEDURES}

\section{OBJECTIVES}

The objective of the verification was to confirm that the remedial action reduced radioactivity and beryllium levels to within the DOE guidelines. Beryllium concentration limits and applicable DOE guidelines for protection against radiation are shown in Table 1. Typical background values for radiation in the Schenectady area are shown in Table 2.

\section{SURVEY METHODS}

The post-remedial action survey was performed on this property as described for a generic site. 5 This survey consists of surface-level alpha and beta-gamma measurements, surface and $1 \mathrm{~m}$ gamma measurements, smears, and systematic soil and miscellaneous debris sampling.

The following procedures were performed to assess the success of the cleanup activities with respect to beryllium and radiological residuals. 


\section{Radiological Survey}

The verification survey included:

-direct alpha and beta-gamma radiation levels taken in each area being smeared or sampled during the beryllium characterization;

-alpha and beta-gamma scans of indoor structural areas, including areas previously identified as above guidelines or previously inaccessible;

-gamma exposure rates at ground level and at 1 -meter from the surface both inside and outside the building;

-collection and analysis of smears taken from selected areas inside the structure to determine radionuclide content; and

-collection and analysis of soil samples taken from areas outside around the rotoclone stack east of the building, and in the vault where the concrete floor was removed.

Indoors, radiological measurements included gamma exposure rates and directly measured alpha and beta-gamma surface activity levels taken wherever smear/sampling was done. Smears and/or samples of dust and debris were collected at systematic locations, including in selected sections of sanitary and storm drain lines, boilers, and stack interiors, to determine transferable and fixed radiation levels. Drain lines were also scanned for betagamma activity to the point of absolute refusal. Samples of brick from outside the facility stack were collected and analyzed to establish a background reference. Background concentrations have not been subtracted from the analytical results.

Outdoors, a portable sodium iodide gamma scintillation instrument was used to obtain ranges of measurements for areas of the property surface and at one meter above the surface. Surface and subsurface soil samples were systematically collected and analyzed from the adjacent fenced-in area on the east side of the building containing the rotoclone stack (see Figs. 1 and 2).

The collection locations of smears and dust and soil samples are indicated on Figs 2-8. Results and analyses are shown in Tables 3 and 4. Table 5 has more detailed comments on some of the samples. Because characterization and verification activities were often carried out concurrently but in different locations, sample numbers are not in consecutive order.

\section{Beryllium Survey}

The verification survey included:

- visual inspections for dust or debris on all surfaces;

- systematic collection and analysis of wet and dry smears and/or samples of dust and debris on horizontal and other structure surfaces such as walls, ceilings, floors, bridging (cross bracing) and stringers (rafters), beams, trusses, cables, pipes, window ledges, light fixtures, and air moving equipment;

- collection and analysis of smears and samples from selected sections of sanitary and storm drain lines and stack interiors; and 
- collection and analysis of samples from underlying materials of the roof and any other areas previously above guidelines or inaccessible.

The method of sample collection on the hard surfaces inside the building was dependent on the surface loading. Surfaces were wiped with dry smears, or the smear paper was moistened with distilled water prior to wiping the surface. A relatively dustfree surface was sampled according to the procedure described in the Department of Energy's (DOE) Environmental Implementation Guide for Radiological Survey Procedures, Draft, November 1992. The analytical results for these samples were then used to calculate beryllium concentration per area $\left(\mu \mathrm{g} / \mathrm{ft}^{2}\right)$ or per mass $(\mu \mathrm{g} / \mathrm{g})$ for comparison with the established site-specific guideline value. Some areas were cleaned and smeared several times before surfaces were visibly clean and all measurements were less than guideline values. Radiological measurements were also performed on all surfaces within the building prior to collecting beryllium smears or samples.

\section{VERIFICATION SURVEY AND ANALYSIS}

Typical background radiation levels for the Schenectady, New York, area are listed in Table 2. The data are provided for purposes of comparison with the survey results presented in this section.

Direct measurement results presented in this report are gross readings; background radiation levels have not been subtracted. Similarly, background radiation levels have not been subtracted from radionuclide concentrations measured in environmental samples.

\section{RADIOLOGICAL SURVEY RESULTS}

Outdoors, gamma exposure rates taken at the ground surface in the area of the rotoclone exhaust stack (adjacent to the east side of the building, Fig. 1) ranged from 14 to $16 \mu \mathrm{R} / \mathrm{h}$, a range comparable to typical background levels recorded for the Schenectady area of 5 to $14 \mu \mathrm{R} / \mathrm{h}$. Gamma exposure rates taken throughout the outdoor areas during the previous characterization surveys were all comparable to typical background levels for the area, with the exception of the rotoclone area where elevated radiological and beryllium levels were initially found. The area has since been excavated and remediated.

In the rotoclone area, surface and subsurface soil samples were taken systematically, using the grid lines drawn by ANL, and analyzed for radionuclide concentrations. After complete remediation, the maximum concentrations of $226 \mathrm{Ra}$ and $232 \mathrm{Th}$ in both surface and subsurface soil were $1.1 \mathrm{pCi} / \mathrm{g}$ and $1.5 \mathrm{pCi} / \mathrm{g}$, respectively. These results approximate average background concentrations (Table 2). Applying the principles of ALARA (As Low As Reasonably Achievable), the maximum concentrations of $238 \mathrm{U}$ in surface and subsurface samples following remediation were 3.6 and $2.6 \mathrm{pCi} / \mathrm{g}$, respectively. These values are well below the $35 \mathrm{pCi} / \mathrm{g}$ guideline for $238 \mathrm{U}$ that was derived for this site (see

Table 1). Sample locations are shown in Fig. 2. One soil sample was also taken from 
beneath the concrete floor in the vault (Fig. 4), after the entire floor was remediated by scabbling. Analysis of the soil showed background levels of $226 \mathrm{Ra}$ and ${ }^{232} \mathrm{Th}$, and a maximum of $10 \mathrm{pCi} / \mathrm{g}$ of $\mathrm{U} 238$, which is well below DOE guidelines. Results of the analyses are listed in Table 3.

Indoors, directly measured beta-gamma levels were checked on each structure wherever smears/samples were taken for beryllium analysis. Most suitably smooth surfaces were also checked for directly measured alpha radiation. All of these measurements were below the minimum detectable activity (MDA) ${ }^{* *}$ for directly measured alpha and beta-gamma levels. In addition, all smears analyzed for transferable alpha and beta-gamma activity were also less than MDA. The general locations of these samples are shown on Fig. 8.

The characterization and supplementary survey reports 2,3 indicated elevated betagamma radiation levels inside the building in drains and drain lines, the former "chem area," and floor of the vault. Additionally, radiological measurements and samples containing localized radionuclide concentrations (i.e., concentrations of $238 \mathrm{U}$ ) higher than the site-specific guidelines were found in the vault, stack thimble in the boiler room, drains and drain lines, and the original floor of Bay 1-2. These areas were remediated by KAPL and scanned and sampled again by ORNL for verification. Final radiological measurements were well below DOE guidelines.

\section{BERYLLIUM SURVEY RESULTS}

Beryllium sampling and analysis done in the previous characterization surveys showed that scattered locations in indoor areas contained elevated levels of beryllium. These areas were located mainly in the overhead structures and in Bays 22-23 through 2728. After complete remediation by KAPL, verification samples and smears were taken by ORNL to determine the effectiveness of the remediation.

The final measurements for beryllium taken in these areas were all well below the established guidelines for this site. Results are shown in Tables 4 and 5 listed alphanumerically by sample number. Table 5 is a listing of samples with more detailed comments. Locations of the samples/smears can be found on Figs. 3-8, depending on whether they are floor-level, drain, horizontal surface, or upper level samples.

\section{OVERCHECKS BY NEW YORK STATE}

Representatives of the New York State Department of Environmental Conservation Bureau of Radiation conducted additional independent overcheck surveys on December 23

\footnotetext{
${ }^{* *}$ The MDA for directly measured alpha levels and beta-gamma dose rates is $25 \mathrm{dpm} / 100 \mathrm{~cm} 2$ and $0.01 \mathrm{mrad} / \mathrm{h}$, respectively. The MDA for transferable alpha activity is $10 \mathrm{dpm} / 100 \mathrm{~cm} 2$, and $200 \mathrm{dpm} / 100$ $\mathrm{cm} 2$ for transferable beta-gamma activity.
} 
1993 for residual radioactivity. The results of these surveys showed no radioactivity above natural background levels. 6

\section{CONCLUSION}

Results of this independent radiological and beryllium verification survey at 425 Peek Street show no evidence for the presence of elevated levels of radioactive residuals indoors or outdoors on the property. Radiation levels, concentrations of radionuclides, and nonradioactive, elemental beryllium in samples and smears were all below guidelines formulated for the site.

Based on all data collected, the industrial property at 425 Peek Street and the adjacent state-owned bike path in Schenectady, New York, conforms to all applicable nonradioactive beryllium and radionuclide guidelines established for this site.

\section{REFERENCES}

1. W. D. Cottrell, R. D. Foley, and R. F. Carrier, Preliminary Site Survey Report For The Peek Street Site, Schenectady, New York, Ornl/Rasa-88/101, Martin Marietta Energy Systems, Inc., Oak Ridge Natl. Lab., March 1989.

2. R. D. Foley, W. D. Cottrell, and R. F. Carrier, Radiological Survey Results for the Peek Street Site Properties, Schenectady, New York, ORNL-6623, Martin Marietta Energy Systems, Inc., Oak Ridge Natl. Lab., August 1992.

3. R. D. Foley, J. F. Allred, R. F. Carrier, Supplementary Radiological and Beryllium Characterization of the Facility at 425 Peek Street Schenectady, New York, ORNL-6623/Supplement, Martin Marietta Energy Systems, Inc., Oak Ridge Natl. Lab., September 1994.

4. Knolls Atomic Power Laboratory, Inc., Post Remedial Action Report for the Peek Street Site Property in Schenectady, New York, KAPL-4803, August 1994.

5. T. E. Myrick, B. A. Berven, W. D. Cottrell, W. A. Goldsmith, and F. F. Haywood, Procedures Manual for the ORNL Radiological Survey Activities (RASA) Program, ORNL/TM-8600, Martin Marietta Energy Systems, Inc., Oak Ridge Natl. Lab., April 1987.

6. New York State Department of Environmental Conservation, correspondence from P. J. Merges to A. R. Seepo, U.S. Department of Energy, Radiological/Environmental Control and Safety Division, Schenectady, New York, February 7, 1994. 


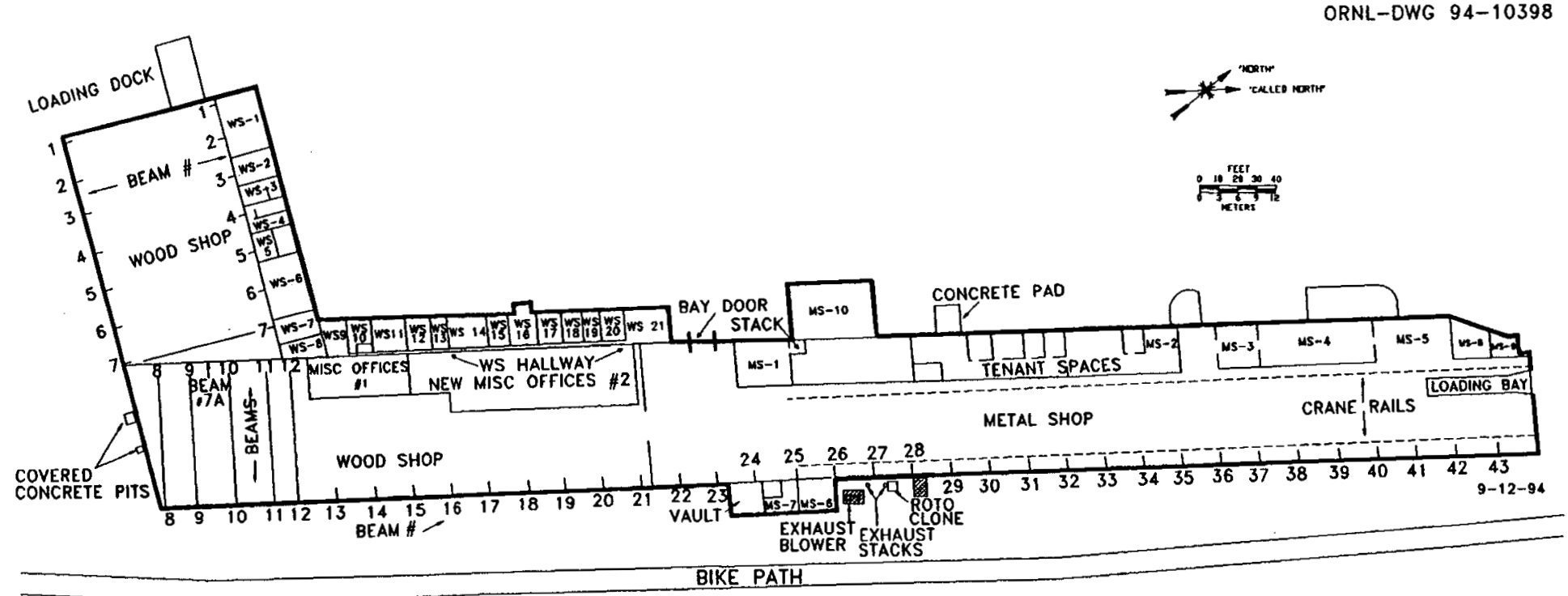

Fig. 1. Diagram showing the layout of the factory building on the industrial property at 425 Peek Street, Schenectady, New York. 


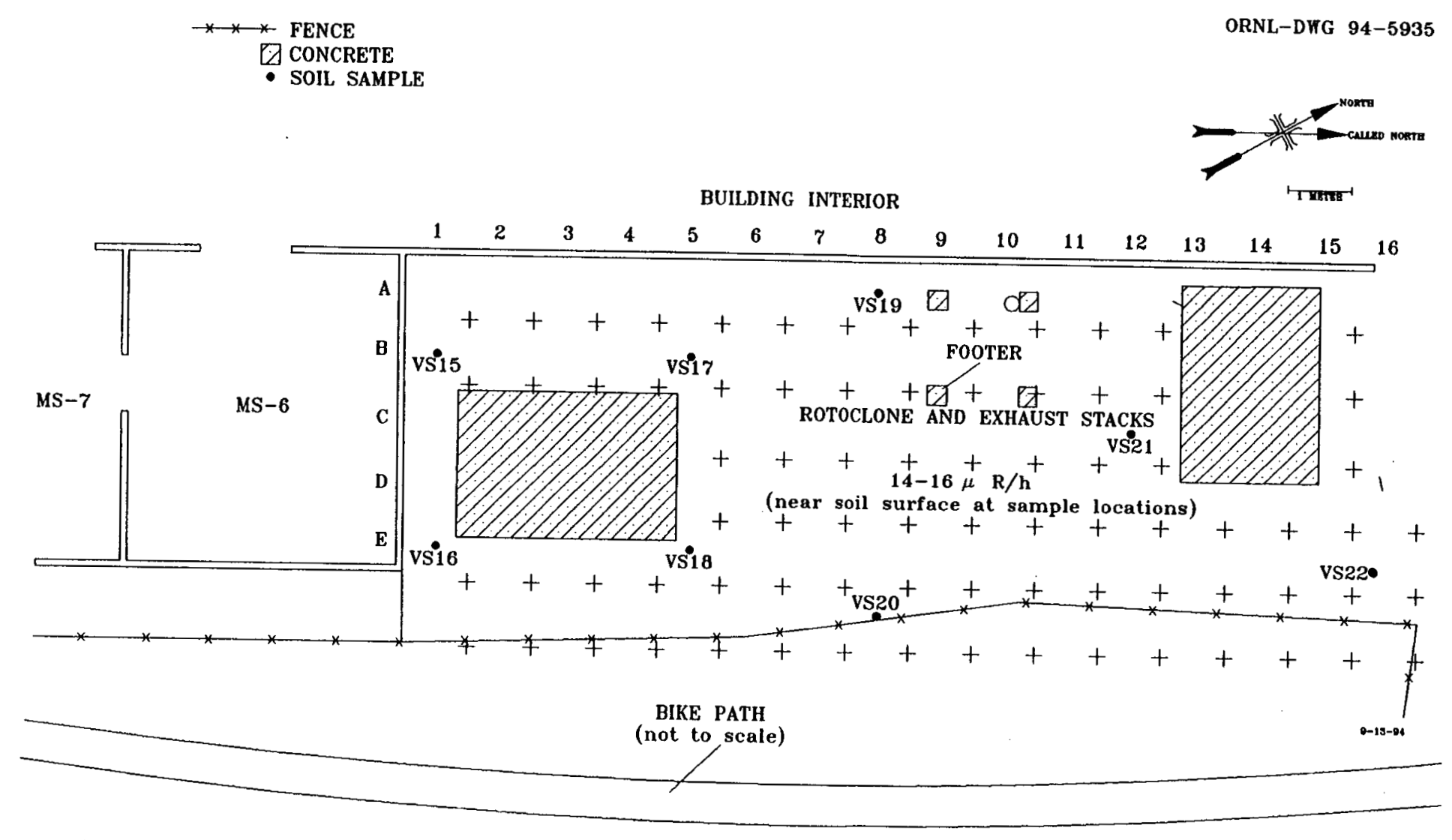

Fig. 2. Diagram showing gamma measurements and soil sampling locations in the gridded area on the east side of the factory building at 425 Peek St., Schenectady, New York. 
RADIOLOGICAL AND BERYLLIUM VERIFICATION SURVEY OF DRAIN SYSTEM

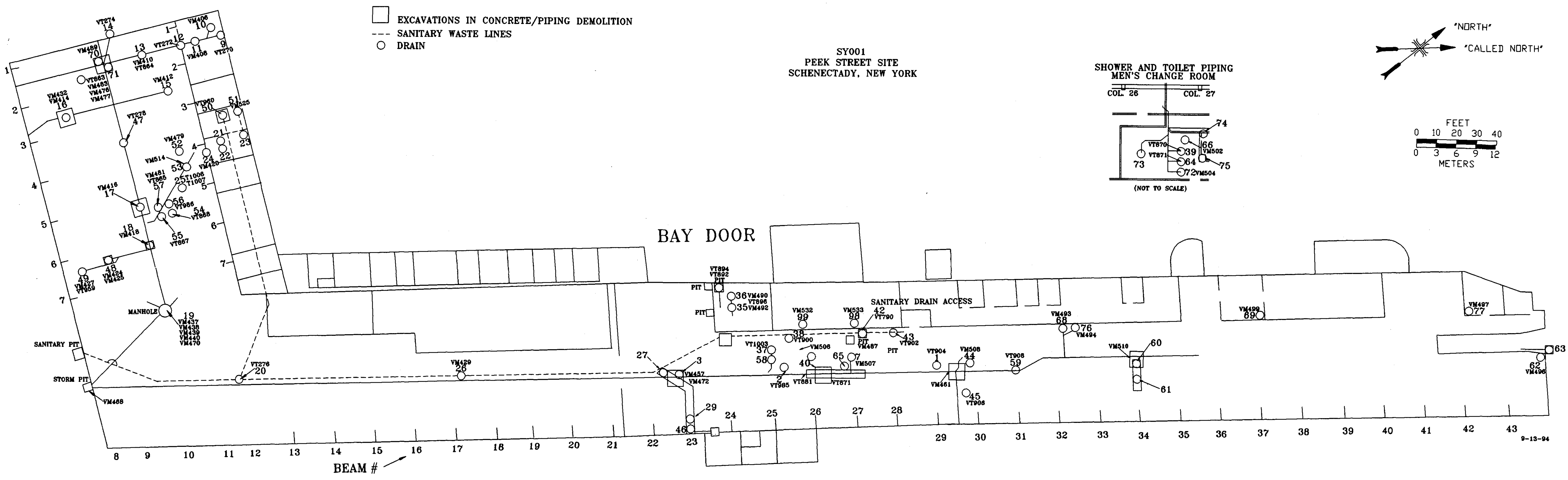

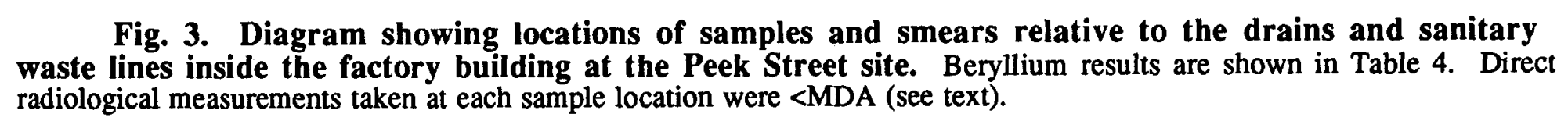




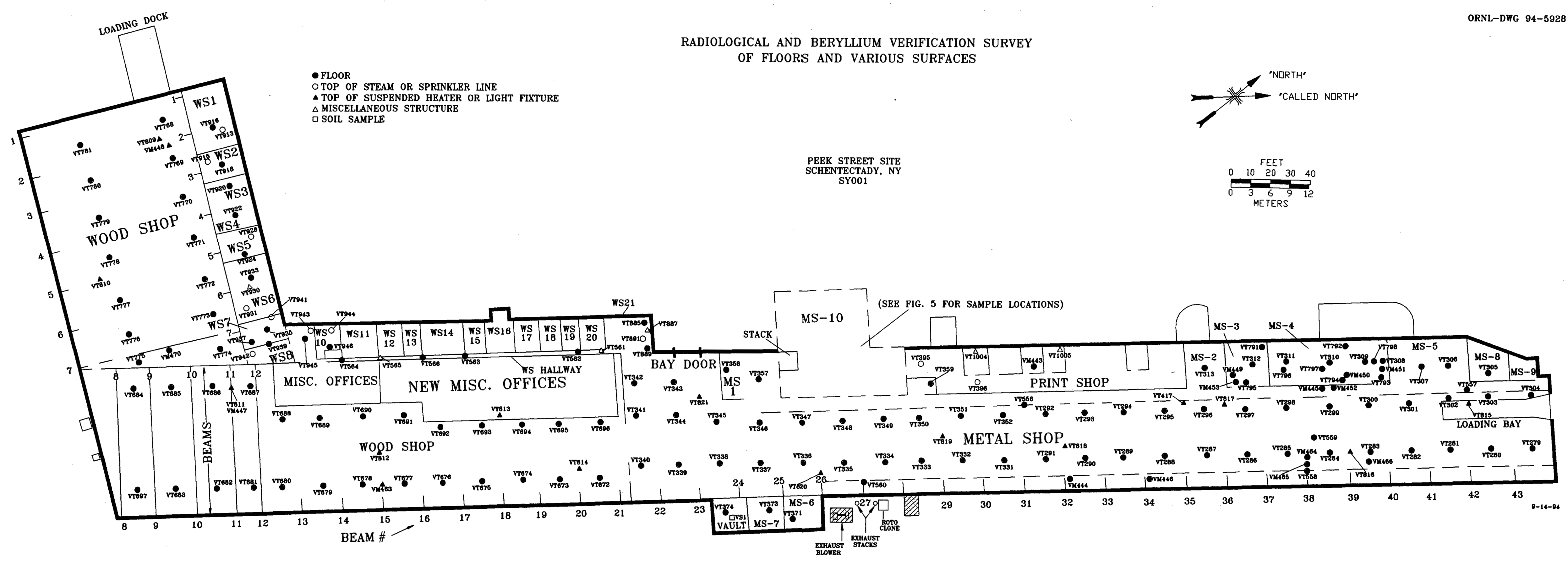




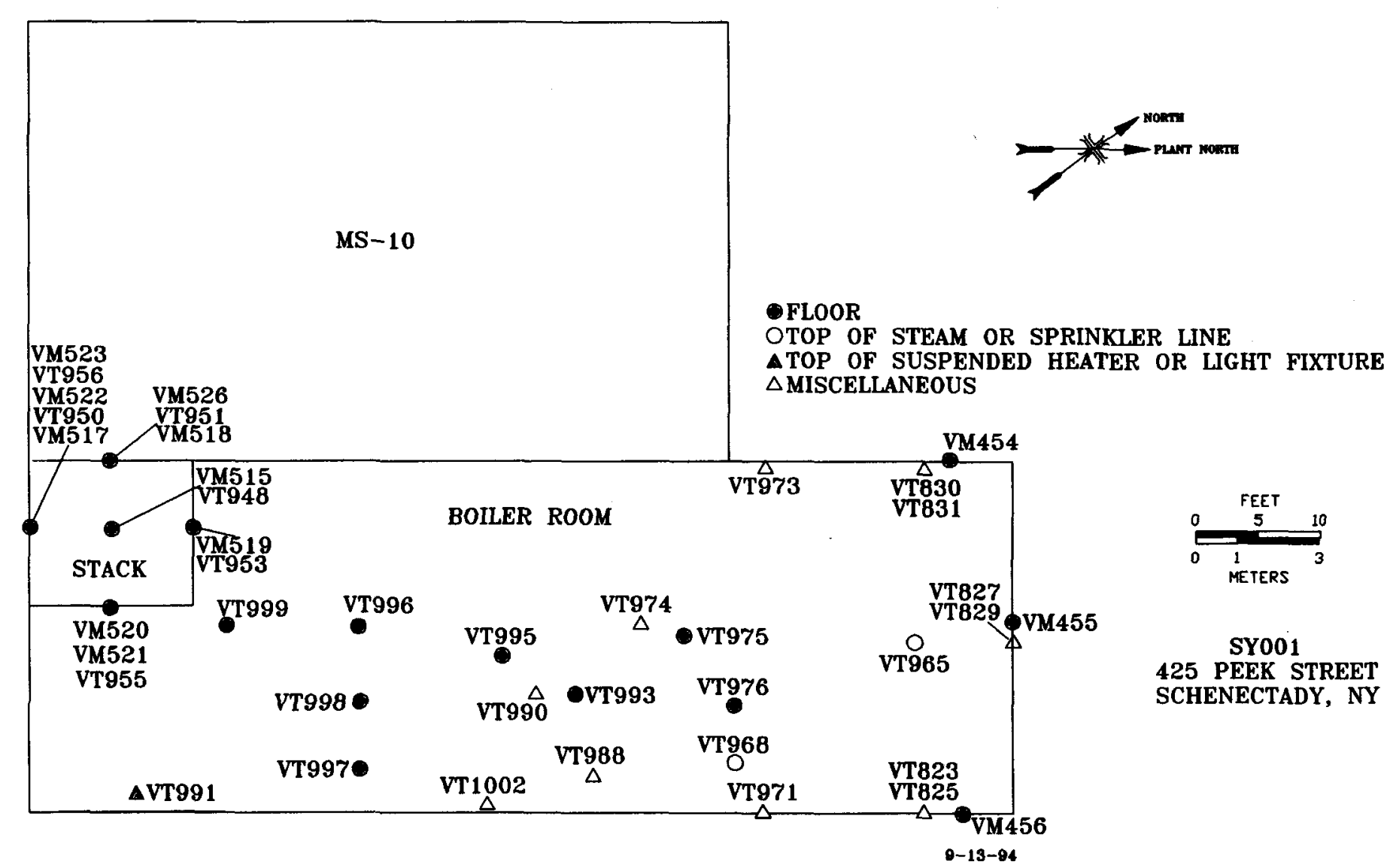

Fig. 5. Close up diagram of room MS-10 and adjacent boiler room showing locations of samples and smears taken from the floor and miscellaneous horizontal surfaces. Beryllium results are shown in Table 4. Direct radiological measurements taken at each sample location were <MDA (see text). 


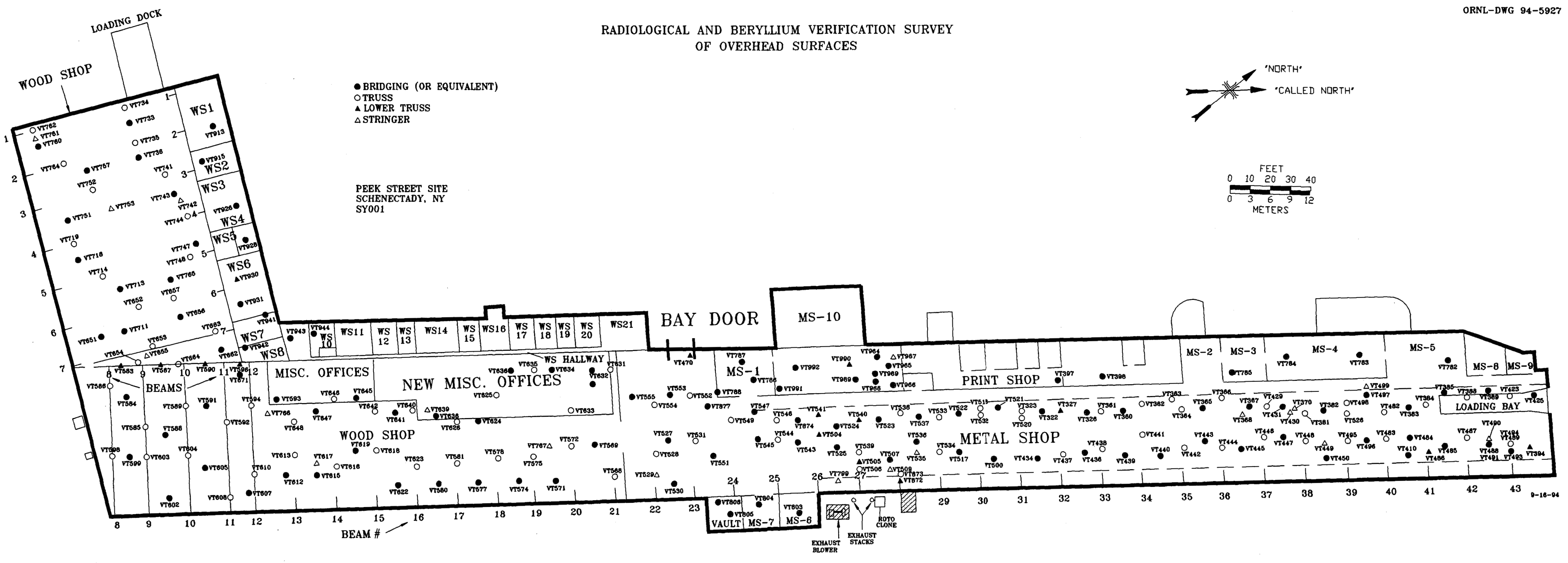

Fig. 6. Diagram showing the locations of samples and smears taken from overhead surfaces in the factory building at the Peek Street site. Beryllium results are shown in Table 4. Direct radiological measurements 
RADIOLOGICAL AND BERYLLIUM VERIFICATION SURVEY OF HORIZONAL SURFACES

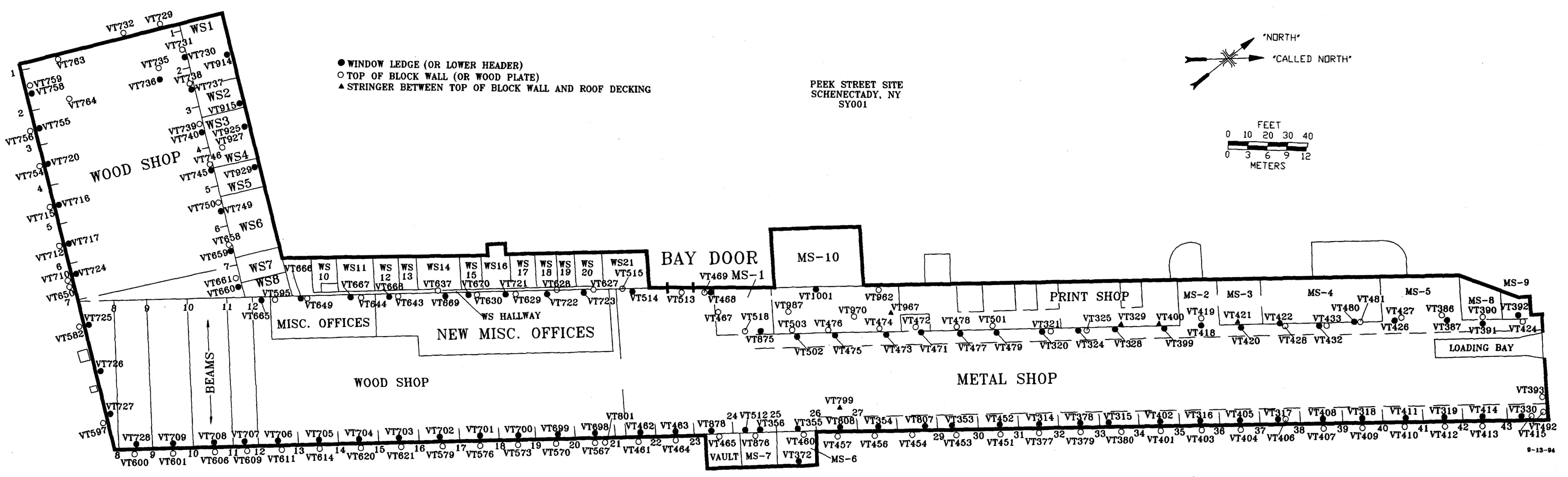


VERIFICATION RADIOLOGICAL ANALYSIS OF SMEARS [SMEARS WERE ALL $<$ MDA]

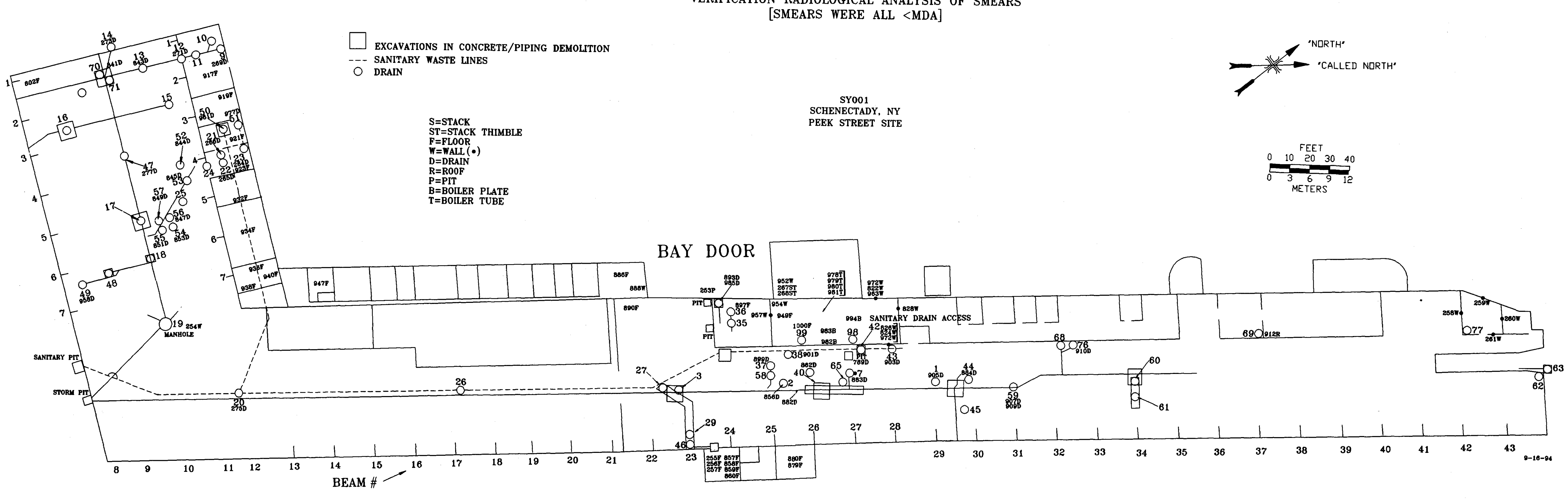


Table 1. Applicable Guidelines for the Peek Street site

\begin{tabular}{|c|c|}
\hline Mode of exposure & Guideline value $a$ \\
\hline $238 \mathrm{U}$ in soil & $35 \mathrm{pCi} / \mathrm{g}$ \\
\hline $\begin{array}{l}238 \mathrm{U} \text { in indoor dust, } \\
\text { removable alpha activity }\end{array}$ & $1000 \mathrm{dpm} / 100 \mathrm{~cm}^{2}$ \\
\hline $\begin{array}{l}238 \mathrm{U} \text { indoors as fixed plus } \\
\text { removable alpha surface } \\
\text { contamination }\end{array}$ & $5000 \mathrm{dpm} / 100 \mathrm{~cm}^{2}$ \\
\hline $238 \mathrm{U}$ in air & $2 \times 10^{-12} \mu \mathrm{Ci} / \mathrm{mL}$ \\
\hline $\begin{array}{l}\text { Beta-gamma } \\
\text { dose rate at } 1 \mathrm{~cm} \text { from } \\
\text { the surface averaged over } \\
\text { not more than } 1 \mathrm{~m}^{2} \text { area }\end{array}$ & $0.2 \mathrm{mrad} / \mathrm{h}$ \\
\hline $\begin{array}{c}\text { Maximum beta-gamma } \\
\text { dose rate at } 1 \mathrm{~cm} \text { from } \\
\text { the surface over any } \\
100-\mathrm{cm}^{2} \text { area }\end{array}$ & $1.0 \mathrm{mrad} / \mathrm{h}$ \\
\hline $\begin{array}{l}\text { Gamma exposure rate } \\
\text { indoors at } 1 \mathrm{~m} \text { above the } \\
\text { surface above background }\end{array}$ & $20 \mu \mathrm{R} / \mathrm{h}$ \\
\hline $\begin{array}{l}\text { Beryllium in removable } \\
\text { surface dust }\end{array}$ & $2 \mu \mathrm{g} / \mathrm{ft}^{2}$ \\
\hline $\begin{array}{l}\text { Beryllium in air } \\
\text { - 8-hr average } \\
\text { - acceptable ceiling } \\
\text { - never to be exceeded }\end{array}$ & $\begin{array}{c}2 \mu \mathrm{g} / \mathrm{m}^{3} \\
5 \mu \mathrm{g} / \mathrm{m}^{3} \\
25 \mu \mathrm{g} / \mathrm{m}^{3}\end{array}$ \\
\hline Beryllium in soil & $13 \mu \mathrm{g} / \mathrm{g}$ \\
\hline${ }^{137} \mathrm{Cs}$ in soil & $34 \mathrm{pCi} / \mathrm{g}$ \\
\hline
\end{tabular}

aSee R. D. Foley et. al., ORNL-6623/Supplement. ${ }^{3}$ 
Table 2. Background radiation levels and concentrations of selected radionuclides in soil samples taken in the Schenectady and Glenville, New York area
Type of radiation measurement or sample

Radiation level or radionuclide concentration ${ }^{a}$

Range Average

Gamma exposure rate at ground surface $(\mu R / h)^{\mathbf{a}}$

10

Gamma exposure rate at $1 \mathrm{~m}$ above

$5-14$

9.5

floor or ground surface $(\mu \mathrm{R} / \mathrm{h})^{\mathrm{b}}$

Concentration of radionuclides

in soil ( $\mathrm{pCi} / \mathrm{g}$ dry wt $)^{a}$

$226 \mathrm{Ra}$

$0.83-0.98$

0.93

$232 \mathrm{Th}$

$0.81-0.95$

0.89

$238 \mathrm{U}$

$0.94-1.3$

1.2

$a$ Values and soil samples (SY002S75-SY002S77) were obtained from three undisturbed locations near the Sacandaga site in Glenville. ${ }^{2}$

$b$ Background at Schenectady determined in 1982 by EG\&G over $260 \mathrm{~km}^{2}$ area. 2 
Table 3. Concentrations of radionuclides in soil samples collected for the independent verification of the property at 425 Peek Street, Schenectady, New York

\begin{tabular}{|c|c|c|c|c|c|c|}
\hline \multirow[b]{2}{*}{$\begin{array}{c}\text { Sample } \\
\text { ID }\end{array}$} & \multirow[b]{2}{*}{ Location ${ }^{a}$} & \multirow[b]{2}{*}{$\begin{array}{l}\text { Depth } \\
(\mathrm{cm})\end{array}$} & \multirow[b]{2}{*}{ Comments } & \multicolumn{3}{|c|}{ Radionuclide concentration $(\mathrm{pCi} / \mathrm{g})^{b}$} \\
\hline & & & & $226 \mathrm{Ra}$ & 232Th & $238 \mathrm{U}$ \\
\hline VS1 & Vault & c & From under 8 in. concrete & $0.37 \pm 0.04$ & $0.28 \pm 0.07$ & $10 \pm 2.5$ \\
\hline VS15A & $\mathrm{B} 1 *$ & $0-5$ & 12 in. below excavation & $1.0 \pm 0.11$ & $0.91 \pm 0.07$ & $1.2 \pm 0.43$ \\
\hline VS15B & $\mathrm{B} 1^{*}$ & $5-15$ & 12 in. below excavation & $0.82 \pm 0.06$ & $0.99 \pm 0.14$ & $0.96 \pm 0.50$ \\
\hline VS16A & $\mathrm{E} 1^{*}$ & $0-5$ & 12.5 in. below excavation & $0.43 \pm 0.05$ & $0.52 \pm 0.11$ & $0.93 \pm 0.40$ \\
\hline VS16B & $\mathrm{E} 1^{*}$ & $5-15$ & 12.5 in. below excavation & $0.60 \pm 0.10$ & $0.88 \pm 0.08$ & $0.74 \pm 0.49$ \\
\hline VS17A & B5* & $0-5$ & 15 in. below excavation & $0.74 \pm 0.04$ & $0.69 \pm 0.09$ & $0.96 \pm 0.35$ \\
\hline VS17B & B5* & $5-15$ & 15 in below excavation & $1.0 \pm 0.07$ & $0.96 \pm 0.11$ & $1.1 \pm 0.40$ \\
\hline VS18A & E5* & $0-5$ & 16.5 in. below excavation & $0.98 \pm 0.06$ & $1.1 \pm 0.16$ & $2.8 \pm 0.53$ \\
\hline VS18B & E5* & $5-15$ & 16.5 in. below excavation & $1.1 \pm 0.08$ & $1.5 \pm 0.11$ & $2.6 \pm 0.62$ \\
\hline VS19A & $\mathrm{A} 8^{*}$ & $0-5$ & 21 in. below excavation & $1.1 \pm 0.06$ & $1.3 \pm 0.15$ & $2.6 \pm 0.70$ \\
\hline VS19B & A $8^{*}$ & $5-15$ & 21 in. below excavation & $1.0 \pm 0.07$ & $1.5 \pm 0.10$ & $1.3 \pm 0.56$ \\
\hline VS20A & $\mathrm{F} 8 *$ & $0-5$ & 16 in. below excavation & $0.88 \pm 0.06$ & $0.82 \pm 0.12$ & $0.87 \pm 0.50$ \\
\hline VS20B & F8* & $5-15$ & 16 in. below excavation & $1.0 \pm 0.12$ & $0.81 \pm 0.07$ & $1.4 \pm 0.46$ \\
\hline VS21A & $\mathrm{C} 12^{*}$ & $0-5$ & 18 in. below excavation & $1.1 \pm 0.13$ & $0.91 \pm 0.08$ & $1.6 \pm 0.51$ \\
\hline VS21B & $\mathrm{C} 12^{*}$ & $5-15$ & 18 in. below excavation & $1.1 \pm 0.11$ & $0.96 \pm 0.08$ & $2.0 \pm 0.26$ \\
\hline VS22A & E16* & $0-5$ & 12.5 in. below excavation & $1.1 \pm 0.08$ & $1.2 \pm 0.16$ & $3.6 \pm 0.63$ \\
\hline VS22B & E16* & $5-15$ & Center & $0.82 \pm 0.06$ & $0.85 \pm 0.11$ & $2.5 \pm 0.53$ \\
\hline
\end{tabular}

aAlphanumeric grid designations $\left(^{*}\right)$ refer to an ANL grid established in the former Rotoclone area outside the central-eastem part of the building (Fig. 2).

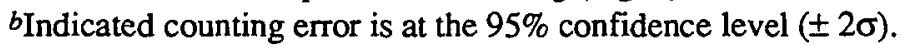

Not applicable. 
Table 4. Alphanumeric listing of samples, locations, and beryllium results for the independent verification survey of $\mathbf{4 2 5}$ Peek Street

\begin{tabular}{|c|c|c|c|c|c|}
\hline $\begin{array}{c}\text { Sample } \\
\mathrm{ID}^{a}\end{array}$ & $\begin{array}{c}\text { Bay/room/ } \\
\text { structure/ } \\
\text { areab }\end{array}$ & $\begin{array}{l}(\mu \mathrm{g} / \mathrm{g}) \\
(\mu \mathrm{g})^{*}\end{array}$ & $\begin{array}{r}\text { Be/area } \\
\left(\mu \mathrm{g} / \mathrm{ft}^{2}\right)^{d} \\
\end{array}$ & $\begin{array}{l}\text { Location } \\
\text { in bay }\end{array}$ & Location notese \\
\hline VM406 & Drain 10 & 0.99 & $f$ & Drain & $\begin{array}{l}\text { Composite sample in interval } 3 \text { to } 8 \text { in. (involving } \\
\text { walls) \& neck of drain pipe }\end{array}$ \\
\hline VM408 & Drain 11 & 0.76 & $f$ & Drain & All sidewalls samples no horiz. sample \\
\hline VM410 & Drain 13 & 0.67 & $f$ & Drain & 15 in depth sampled $1.5-15$ in. \\
\hline VM412 & Drain 15 & 2.0 & $f$ & Drain & Accessible depth sampled - $10.5 \mathrm{in}$. \\
\hline VM414 & Drain 16 & 0.65 & $f$ & Drain & $\begin{array}{l}\text { Accessible depth sampled - } 12 \text { in. From connecting } \\
\text { neck Pipe A to B }\end{array}$ \\
\hline VM416 & Drain 17 & 0.94 & $f$ & Drain & Drain depth $=6$ in. pipe size $=2$ in. \\
\hline VM418 & Drain 18 & 0.55 & $f$ & Drain & $\begin{array}{l}\text { Sample taken from various locations in pit bottom } \\
\text { drain depth }=32 \mathrm{in} \text {. }\end{array}$ \\
\hline VM420 & Drain 24 & 6.1 & $f$ & Drain & Drain depth $=13$ in. Pipe $A=3$ in. \& Pipe $B=4$ in. \\
\hline VM424 & Drain 48 & 3.6 & $f$ & Drain & $\begin{array}{l}\text { Drain depth }=12 \text { in.; pipe size }=34 \text { in.; liquid present } \\
\text { in drain (oily) }\end{array}$ \\
\hline VM425 & Drain 48 & 0.36 & $f$ & Drain & Oily sludge \\
\hline VM427 & Drain 49 & 0.99 & $f$ & Drain & Drain depth $=11.5$ in.; pipe size 2.5 in. \\
\hline VM429 & Drain 26 & 0.65 & $f$ & Drain & Drain depth $=50$ in. \& pipe size is $3.5 \mathrm{in}$. \\
\hline VM432 & Drain 16 & 1.0 & $f$ & Drain & $\begin{array}{l}\text { From horiz. pipe just } N \text { of Drain } 16 \text { cutout, } \\
\text { previously cleaned out and surveyed } 9 / 16 / 93\end{array}$ \\
\hline VM437 & Drain 19 & 0.35 & $f$ & Drain & Composite sample from bottom of manhole sump \\
\hline VM438 & Drain 19 & 0.27 & $f$ & Drain & Core of sludge from the manhole sump; KAPL no. 1 \\
\hline VM439 & Drain 19 & 0.34 & $f$ & Drain & Core of sludge from the manhole sump; KAPL no. 2 \\
\hline VM440 & Drain 19 & 0.27 & $f$ & Drain & Core of sludge from the manhole sump; KAPL no. 3 \\
\hline VM443 & $31 \mathrm{~W}$ & 0.47 & $f$ & Floor & Floor $7 \mathrm{ft} E \& 3 \mathrm{ft} 4$ in. $N$ of Col. $31 \mathrm{~W}$ \\
\hline VM444 & $32 \mathrm{E}$ & 0.55 & $f$ & Floor & $6 \mathrm{in.} W \& 1 \mathrm{ft} 7$ in. $N$ of Col. $32 \mathrm{E}$ \\
\hline VM445 & $38 \mathrm{~W}$ & 0.49 & $f$ & Floor & $20 \mathrm{ft} \& 11 \mathrm{in} . \mathrm{E}$ and $5 \mathrm{ft} \& 3 \mathrm{in} . \mathrm{N}$ of Col. $38 \mathrm{~W}$ \\
\hline VM446 & $34 \mathrm{E}$ & 0.39 & $f$ & Floor & 4 ft 10 in. $N \& 1 \mathrm{ft} 11$ in. W of Col. $34 \mathrm{E}$ \\
\hline VM447 & $10-11 \mathrm{~W}$ & 1.9 & $f$ & Other & $\begin{array}{l}\text { Light fixture on Truss } 11,13 \text { of the way (east) over } \\
\text { towards peak }\end{array}$ \\
\hline VM448 & $2-3 N$ & 1.4 & $f$ & Other & $\begin{array}{l}\text { Light fixture on Truss } 2,14 \text { of the way over (south) } \\
\text { towards peak }\end{array}$ \\
\hline VM449 & $36 \mathrm{~W}$ & 0.85 & $f$ & Floor & $\begin{array}{l}\text { Original floor (under styrofoam) in refrig. room } 20 \mathrm{ft} \\
\mathrm{W} \& 20 \mathrm{ft} \mathrm{N} \text { of Col. } 36 \mathrm{~W}\end{array}$ \\
\hline VM450 & $39 \mathrm{~W}$ & 0.40 & $f$ & Floor & $\begin{array}{l}\text { Original floor (under styrofoam) in refrigeration room } \\
20 \mathrm{ft} W \text { of Col. } 39 \mathrm{~W}\end{array}$ \\
\hline
\end{tabular}


Table 4. (continued)

\begin{tabular}{|c|c|c|c|c|c|}
\hline $\begin{array}{c}\text { Sample } \\
\text { ID } a\end{array}$ & $\begin{array}{c}\text { Bay/room/ } \\
\text { structure/ } \\
\text { area }^{b}\end{array}$ & $\begin{array}{c}\mathrm{Be}^{c} \\
(\mu \mathrm{g} / \mathrm{g}) \\
(\mu \mathrm{g})^{*}\end{array}$ & $\begin{array}{c}\text { Be/area } \\
\left(\mu \mathrm{g} / \mathrm{ft}^{2}\right)^{d} \\
\end{array}$ & $\begin{array}{l}\text { Location } \\
\text { in bay }\end{array}$ & Location notese \\
\hline VM451 & $40 \mathrm{~W}$ & 0.75 & $f$ & Floor & $\begin{array}{l}\text { Original floor (under styrofoam) in refrig. rm. } 2 \mathrm{ft} \mathrm{W} \\
\& 4 \mathrm{ft} \mathrm{S} \text { of Col. } 40 \mathrm{~W}\end{array}$ \\
\hline VM452 & $39 \mathrm{~W}$ & 0.30 & $f$ & Floor & $\begin{array}{l}\text { Original floor (under styrofoam) in refrig. rm. } 6 \mathrm{ft} \mathrm{S} \\
\& 2 \mathrm{ft} \mathrm{W} \text { of Col. } 39 \mathrm{~W}\end{array}$ \\
\hline VM453 & $36 \mathrm{~W}$ & 0.70 & $f$ & Floor & $\begin{array}{l}\text { Original floor (under styrofoam) in refrig. } \mathrm{rm} .8 \mathrm{ft} \mathrm{N} \\
\& 2 \mathrm{ft} W \text { of Col. } 36 \mathrm{~W}\end{array}$ \\
\hline VM454 & $28 \mathrm{~W}$ & 1.4 & $f$ & Wall & $\begin{array}{l}\text { Boiler room sand pit, } 6.5 \mathrm{ft} \mathrm{S} \mathrm{\&} 19 \mathrm{ft} \mathrm{W} \text { of Col. } 28 \mathrm{~W} \\
\text { (west outside wall of bldg.) }\end{array}$ \\
\hline VM455 & $28 \mathrm{~W}$ & 1.3 & $f$ & Wall & $\begin{array}{l}\text { Boiler room sand pit, } 11 \mathrm{ft} \mathrm{W} \text { of Col. } 28 \mathrm{~W} \text {, north } \\
\text { wall }\end{array}$ \\
\hline VM456 & $28 \mathrm{~W}$ & 1.2 & $f$ & Wall & Boiler room sand pit, $6 \mathrm{ft} \mathrm{S}$ of Col. $28 \mathrm{~W}$, east wall \\
\hline VM457 & Drain 4 & $<1.0$ & $f$ & Drain & $\begin{array}{l}\text { Drain } 3 \text { at } 26 \mathrm{ft} \mathrm{W} \mathrm{\&} 4 \mathrm{ft} \mathrm{S} \text { of Col. 23E; sample } \\
\text { aliquoted for ORNL (VM458) }\end{array}$ \\
\hline VM461 & Drain & 1.5 & $f$ & Drain & $\begin{array}{l}\text { Drainline at } 28 \mathrm{ft} \mathrm{W} \& 11 \mathrm{Ft} \text { S of Col. 30E; sample } \\
\text { aliquoted for ORNL (VM462) }\end{array}$ \\
\hline VM463 & $15-16 E$ & 1.5 & $f$ & Other & $\begin{array}{l}\text { Light fixture } 15 \mathrm{ft} W \text { from } E \text { wall at Col. } 15 \mathrm{E} \text {. } \\
\text { Material could possibly be dust. }\end{array}$ \\
\hline VM464 & $38 \mathrm{~W}$ & 0.30 & $f$ & Floor & $\begin{array}{l}\text { Original floor after styrofoam \& concrete removed in } \\
\text { refrig. room near Col. } 38 \mathrm{~W}\end{array}$ \\
\hline VM465 & $38 \mathrm{~W}$ & 0.40 & $f$ & Floor & $\begin{array}{l}\text { Original floor after removal of top concrete \& } \\
\text { styrofoam in refrig. rm.near Col. } 38 \mathrm{~W}\end{array}$ \\
\hline VM466 & $39 \mathrm{~W}$ & 0.80 & $f$ & Floor & $\begin{array}{l}\text { Refrig. rm original floor after removal of top concrete } \\
\& \text { styrofoam near Col. } 39 \mathrm{~W}\end{array}$ \\
\hline VM468 & $8 \mathrm{E}$ & 1.2 & $f$ & Floor & $\begin{array}{l}\text { Storm sewer basin floor about } 30 \mathrm{ft} \mathrm{W} \text { of Col. } 8 \mathrm{E} \& \\
10 \mathrm{ft} \mathrm{S} \text { of Col. line } 8\end{array}$ \\
\hline VM470 & Wood shop & 1.2 & $f$ & Drain 19 & Sludge sample from bottom of manhole in wood shop \\
\hline VM472 & $23 E$ & $<3.0$ & $f$ & Drain & $\begin{array}{l}\text { Inside drain pipe (lateral line running just } \mathrm{N} \text { of } \mathrm{Col} \text {. } \\
\text { 23E) access opening }\end{array}$ \\
\hline VM476 & $2 S$ & 1.0 & $f$ & Drain & Drain debris at $41 \mathrm{ft} N \& 9.5 \mathrm{ft} \mathrm{W}$ of Col. $2 \mathrm{~S}$. \\
\hline VM477 & $2 S$ & 1.0 & $f$ & Drain & $\begin{array}{l}\text { Horizontal run debris } 35.5 \mathrm{ft} \mathrm{N} \& 9.5 \mathrm{ft} \mathrm{W} \text { of Col. } \\
\text { 2S. Disposed. }\end{array}$ \\
\hline VM479 & Drain 52 & 1.0 & $f$ & Drain & Drain $5210 \mathrm{ft} \mathrm{S}$ of Col. $4 \mathrm{~N}$ in line with Col. \\
\hline VM481 & Drain 57 & 1.0 & $f$ & Drain & Drain 57 at $36 \mathrm{ft} \mathrm{S} \mathrm{\&} 7 \mathrm{ft} \mathrm{E}$ of Col. $5 \mathrm{~N}$ \\
\hline VM483 & Drain 71 & 1.0 & $f$ & Drain & Material from drain at $38 \mathrm{ft} \mathrm{N} \& 8.5 \mathrm{ft} \mathrm{W}$ of Col. $2 \mathrm{~S}$ \\
\hline VM487 & Drain & 1.0 & $f$ & Drain & Drainline $30 \mathrm{ft} \mathrm{W} \& 1 \mathrm{ft} \mathrm{S}$ of Col. $27 \mathrm{E}$ \\
\hline VM489 & Drain & 1.0 & $f$ & Drain & $\begin{array}{l}\text { Drainline (to be assigned) at } 38 \mathrm{ft} \mathrm{N} \& 11.5 \mathrm{ft} \mathrm{W} \text { of } \\
\text { Col. } 2 \mathrm{~S}\end{array}$ \\
\hline
\end{tabular}


Table 4. (continued)

\begin{tabular}{|c|c|c|c|c|c|}
\hline $\begin{array}{l}\text { Sample } \\
\text { ID } a\end{array}$ & $\begin{array}{c}\text { Bay/room/ } \\
\text { structure/ } \\
\text { area }^{b}\end{array}$ & $\begin{array}{c}\mathrm{Be}^{c} \\
(\mu \mathrm{g} / \mathrm{g}) \\
(\mu \mathrm{g})^{*}\end{array}$ & $\begin{array}{c}\text { Be/area } \\
\left(\mu \mathrm{g} / \mathrm{ft}^{2}\right)^{d} \\
\end{array}$ & $\begin{array}{l}\text { Location } \\
\text { in bay }\end{array}$ & Location notes ${ }^{e}$ \\
\hline VM490 & Drain 36 & 1.0 & $f$ & Drain & $\begin{array}{l}\text { Scooped from debris at } 16 \text { in. depth of floor drain } 4 \mathrm{ft} \\
\mathrm{N} \& 8 \mathrm{ft} \text { E of Col. } 24 \mathrm{~W}\end{array}$ \\
\hline VM492 & Drain 35 & 1.0 & $f$ & Drain & $\begin{array}{l}\text { Drain pipe composite at all depths to } 24 \text { in. at } 4 \mathrm{ft} N \\
\& 10.5 \mathrm{ft} \mathrm{E} \text { of Col. } 24 \mathrm{~W}\end{array}$ \\
\hline VM493 & Drain 68A & 1.0 & $f$ & Drain & $\begin{array}{l}\text { Horizontal drain pipe scoop (bottom radius along } \\
\text { entire } 12 \text { in. length) at Col. } 32 \mathrm{~W}\end{array}$ \\
\hline VM494 & Drain & 1.0 & $f$ & Drain & $\begin{array}{l}\text { Drain pipe scoop composite (entire sidewall depths to } \\
16 \mathrm{in.} \text { ) } 3 \mathrm{ft} \mathrm{N} \text { of Col. } 32 \mathrm{~W}\end{array}$ \\
\hline VM496 & Drain 63 & 1.0 & $f$ & Drain & $\begin{array}{l}\text { Drain to main trunkline (scooped bottom radius) at } 0 \\
\mathrm{ft} N \& 27 \mathrm{ft} \mathrm{W} \text { of Col. 44E }\end{array}$ \\
\hline VM497 & Drain 77 & 1.0 & $f$ & Drain & $\begin{array}{l}\text { Drain pipe (scooped to depth of } 3 \text { in. to horizontal } \\
\text { access debris) at Col. } 42 \mathrm{~W}\end{array}$ \\
\hline VM499 & Drain 69 & 1.9 & $f$ & Drain & $\begin{array}{l}\text { Roof drain } 2 \text { scoop (to depth of } 3.5 \mathrm{ft} \text { ) at } 0 \mathrm{ft} N \& 18 \\
\mathrm{ft} E \text { of Col. } 37 \mathrm{~W}\end{array}$ \\
\hline VM502 & Drain 66 & 5.0 & $f$ & Drain & $\begin{array}{l}\text { Horizontal drain line opening at } 11 \mathrm{ft} \mathrm{E} \& 14 \mathrm{ft} \mathrm{N} \text { of } \\
\text { Col. } 26 \mathrm{~W}\end{array}$ \\
\hline VM504 & Drain 72 & 1.0 & $f$ & Drain & $\begin{array}{l}\text { Vertical drain opening at } 18 \mathrm{ft} \mathrm{E} \mathrm{\&} 12 \mathrm{ft} \mathrm{N} \text { of Col. } \\
26 \mathrm{~W} \text { at floor level }\end{array}$ \\
\hline VM506 & Drain & 1.0 & $f$ & Drain & $\begin{array}{l}\text { Horizontal (lateral) drain line opening at } 12 \mathrm{ft} \& 20 \mathrm{ft} \\
\text { E of Col. } 26 \mathrm{~W} \text {. }\end{array}$ \\
\hline VM507 & Drain 7 & 1.3 & $f$ & Drain & Horizontal drain line opening at $21 \mathrm{ft} E$ of Col. $27 \mathrm{~W}$ \\
\hline VM508 & Drain 44 & 1.0 & $f$ & Drain & $\begin{array}{l}\text { Lateral (horizontal) drain line opening at } 24 \mathrm{ft} \mathrm{W} \& 8 \\
\mathrm{ft} S \text { of Col. } 30 \mathrm{E}\end{array}$ \\
\hline VM510 & Drain & 6.0 & $f$ & Drain & $\begin{array}{l}\text { Horizontal drain line opening located } 18 \mathrm{ft} E \& 1 \mathrm{ft} \mathrm{S} \\
\text { of Col. } 34 \mathrm{~W}\end{array}$ \\
\hline VM514 & Drain 53 & 9.5 & $f$ & Drain & $\begin{array}{l}\text { Cast iron drain pipe (scooped at } 14 \text { in. depth) } 15.33 \mathrm{ft} \\
\mathrm{S} \& 7.5 \mathrm{ft} \mathrm{E} \text { of Col. } 4 \mathrm{~N}\end{array}$ \\
\hline VM515 & MS-1 & 0.46 & $f$ & Stack & $\begin{array}{l}\text { Center of stack floor with sample chiseled from } \\
\text { concrete floor }\end{array}$ \\
\hline VM517 & MS-1 & 0.21 & $f$ & Stack & $\begin{array}{l}\text { Stack S inside wall (fire brick surface) at } 8.5 \mathrm{ft} \text { above } \\
\text { the stack floor level }\end{array}$ \\
\hline VM518 & MS-1 & 0.20 & $f$ & Stack & $\begin{array}{l}\text { Stack west side wall (inside) at } 20 \mathrm{ft} \text { above the stack } \\
\text { floor level }\end{array}$ \\
\hline VM519 & MS-1 & 0.88 & $f$ & Stack & $\begin{array}{l}\text { Stack north inside wall (red brick surface) at } 30 \mathrm{ft} \\
\text { above stack floor level }\end{array}$ \\
\hline VM520 & MS-1 & 0.38 & $f$ & Stack & $\begin{array}{l}\text { Stack east inside wall (red brick surface) at } 40 \mathrm{ft} \text { above } \\
\text { the stack floor level }\end{array}$ \\
\hline
\end{tabular}


Table 4. (continued)

\begin{tabular}{|c|c|c|c|c|c|}
\hline $\begin{array}{l}\text { Sample } \\
\text { ID } a\end{array}$ & $\begin{array}{c}\text { Bay/room/ } \\
\text { structure/ } \\
\text { area }{ }^{b}\end{array}$ & $\begin{array}{c}\mathrm{Be}^{c} \\
(\mu \mathrm{g} / \mathrm{g}) \\
(\mu \mathrm{g})^{*}\end{array}$ & $\begin{array}{c}\text { Be/area } \\
\left(\mu \mathrm{g} / \mathrm{ft}^{2}\right)^{d}\end{array}$ & $\begin{array}{l}\text { Location } \\
\text { in bay }\end{array}$ & Location notese \\
\hline VM521 & MS-1 & 0.54 & $f$ & Stack & $\begin{array}{l}\text { Red brick surface of the stack east inside wall directly } \\
\text { above sample VM } 520\end{array}$ \\
\hline VM522 & MS-1 & 0.36 & $f$ & Stack & $\begin{array}{l}\text { South inside wall (red brick surface) of stack at } 50 \mathrm{ft} \\
\text { above stack floor level }\end{array}$ \\
\hline VM523 & MS-1 & 0.15 & $f$ & Stack & $\begin{array}{l}\text { Fire brick from stack south thimble opening at } 10 \mathrm{ft} \\
\text { above stack floor }\end{array}$ \\
\hline VM525 & Drain 51 & 0.08 & $f$ & Drain & $\begin{array}{l}\text { Sample collected from sidewalls of Drain } 51 \text { (18 in. } \\
\text { depth to elbow) }\end{array}$ \\
\hline VM526 & MS-1 & 1.5 & $f$ & Stack & $\begin{array}{l}\text { Unexposed brick (exposed chiseled away) outside west } \\
\text { wall of stack in MS-10 }\end{array}$ \\
\hline VM528 & Boiler & $<0.10$ & $f$ & Boiler & $\begin{array}{l}\text { Plated deposit (scraped with putty knife; prob. } \\
\text { calcium) from inside surface of boiler plate }\end{array}$ \\
\hline VM529 & Boiler & $<0.10$ & $f$ & Boiler & $\begin{array}{l}\text { Scraping from outside surface of tube (west end boiler) } \\
\text { at } 15 \text { in. from end of tube }\end{array}$ \\
\hline VM532 & $26 \mathrm{~W}$ & 0.12 & $f$ & Drain & $\begin{array}{l}\text { Drain sidewall composite to depth of } 6 \text { in. at } 12 \mathrm{ft} \mathrm{S} \\
\& 4 \mathrm{ft} \mathrm{W} \text { of Col. } 26 \mathrm{~W}\end{array}$ \\
\hline VM533 & Drain 48 & 9.4 & $f$ & Drain & $\begin{array}{l}\text { Drain composite (basin \& partial NW sidewall to a } 5 \\
\text { in. depth) near Col. } 27 \mathrm{~W}\end{array}$ \\
\hline VT268 & MS-1 & $1.1^{*}$ & $f$ & Stack & Sample from stack thimble \\
\hline VT270 & Drain 9 & $0.10 *$ & $f$ & Drain & $\begin{array}{l}\text { Drain } 9 \text { appears to have a } 4 \text { in. diameter; See } \\
\text { additional comments }\end{array}$ \\
\hline VT272 & Drain 12 & $0.10 *$ & $f$ & Drain & "insuff. mat'l for sample" \\
\hline VT274 & Drain 14 & $0.10 *$ & $f$ & Drain & Drain 14 appears to have a 4 in. diameter \\
\hline VT276 & Drain 20 & $0.10 *$ & $f$ & Drain & Drain depth $=50$ in. pipe size $=3.5$ in. \\
\hline VT278 & Drain 47 & $0.10 *$ & $f$ & Drain & $\begin{array}{l}\text { Drain depth }=16 \text { in.; pipe size }=3.5 \text { in.; smear taken } \\
2 \text { E pipe coming into A }\end{array}$ \\
\hline VT279 & NE bldg. & $0.19 *$ & 0.19 & Floor & $9 \mathrm{ft} S \& 14 \mathrm{ft}$ W of NE comer of building \\
\hline VT280 & $43 E$ & $0.15 *$ & 0.15 & Floor & $9 \mathrm{ft} \mathrm{S} \& 14 \mathrm{ft} \mathrm{W}$ of Col. $43 E$ \\
\hline VT281 & $42 \mathrm{E}$ & $0.15^{*}$ & 0.15 & Floor & $9 \mathrm{ft} \mathrm{S} \& 14 \mathrm{ft} \mathrm{W}$ of Col. 42E \\
\hline VT282 & $41 \mathrm{E}$ & $0.23 *$ & 0.23 & Floor & $9 \mathrm{ft} \mathrm{S} \& 14 \mathrm{ft} \mathrm{W}$ of Col. $41 \mathrm{E}$ \\
\hline VT283 & $40 \mathrm{E}$ & $0.19 *$ & 0.19 & Floor & $9 \mathrm{ft} \mathrm{S} \& 14 \mathrm{ft} \mathrm{W}$ of Col. $40 \mathrm{E}$ \\
\hline VT284 & $39 \mathrm{E}$ & $0.24 *$ & 0.24 & Floor & $9 \mathrm{ft} S \& 14 \mathrm{ft} W$ of Col. 39E \\
\hline VT285 & $38 \mathrm{E}$ & $0.29 *$ & 0.29 & Floor & $9 \mathrm{ft} \mathrm{S} \& 14 \mathrm{ft} \mathrm{W}$ of Col. $38 \mathrm{E}$ \\
\hline VT286 & $37 \mathrm{E}$ & $0.27 *$ & 0.27 & Floor & $9 \mathrm{ft} \mathrm{S} \mathrm{\&} 14 \mathrm{ft} \mathrm{W}$ of Col. 37E \\
\hline VT287 & $36 \mathrm{E}$ & $0.26 *$ & 0.26 & Floor & $9 \mathrm{ft} \mathrm{S} \& 14 \mathrm{ft} \mathrm{W}$ of Col. $36 \mathrm{E}$ \\
\hline
\end{tabular}


Table 4. (continued)

\begin{tabular}{|c|c|c|c|c|c|}
\hline $\begin{array}{l}\text { Sample } \\
\text { ID } a\end{array}$ & $\begin{array}{c}\text { Bay/room/ } \\
\text { structure/ } \\
\text { area }^{b}\end{array}$ & $\begin{array}{c}\mathrm{Be}^{c} \\
(\mu \mathrm{g} / \mathrm{g}) \\
(\mu \mathrm{g})^{*}\end{array}$ & $\begin{array}{l}\text { Be/area } \\
\left(\mu \mathrm{g} / \mathrm{ft}^{2}\right)^{d} \\
\end{array}$ & $\begin{array}{l}\text { Location } \\
\text { in bay }\end{array}$ & Location notese \\
\hline VT288 & $35 \mathrm{E}$ & $0.24 *$ & 0.24 & Floor & $9 \mathrm{ft} \mathrm{S} \& 14 \mathrm{ft} \mathrm{W}$ of Col. $35 \mathrm{E}$ \\
\hline VT289 & $34 \mathrm{E}$ & $0.21 *$ & 0.21 & Floor & $9^{\prime} S \& 14^{\prime} W$ of Col. 34E \\
\hline VT290 & $33 \mathrm{E}$ & $0.27 *$ & 0.27 & Floor & $9 \mathrm{ft} \mathrm{S} \& 14 \mathrm{ft} \mathrm{W}$ of Col. 33E \\
\hline VT291 & $32 \mathrm{E}$ & $0.28 *$ & 0.28 & Floor & $9 \mathrm{ft} \mathrm{S} \& 14 \mathrm{ft} \mathrm{W}$ of Col. 32E \\
\hline VT292 & $32 \mathrm{~W}$ & $0.23 *$ & 0.23 & Floor & $9 \mathrm{ft} \mathrm{S} \& 14 \mathrm{ft} \mathrm{E}$ of Col. $32 \mathrm{~W}$ \\
\hline VT293 & $33 \mathrm{~W}$ & $0.27 *$ & 0.27 & Floor & $9 \mathrm{ft} \mathrm{S} \& 14 \mathrm{ft} \mathrm{E}$ of Col. 33W \\
\hline VT294 & $34 \mathrm{~W}$ & $0.21 *$ & 0.21 & Floor & $9 \mathrm{ft} \mathrm{S} \& 14 \mathrm{ft} \mathrm{E}$ of Col. $34 \mathrm{~W}$ \\
\hline VT295 & $35 \mathrm{~W}$ & $0.27 *$ & 0.27 & Floor & $9 \mathrm{ft} \mathrm{S} \& 14 \mathrm{ft} \mathrm{E}$ of Col. $35 \mathrm{~W}$ \\
\hline VT296 & $36 \mathrm{~W}$ & $0.24 *$ & 0.24 & Floor & $9 \mathrm{ft} \mathrm{S} \& 14 \mathrm{ft} \mathrm{E}$ of Col. $36 \mathrm{~W}$ \\
\hline VT297 & $37 \mathrm{~W}$ & $0.22 *$ & 0.22 & Floor & $9 \mathrm{ft} \mathrm{S} \& 14 \mathrm{ft} \mathrm{E}$ of Col. $37 \mathrm{~W}$ \\
\hline VT298 & $38 \mathrm{~W}$ & $0.23 *$ & 0.23 & Floor & $9 \mathrm{ft} \mathrm{S} \& 14 \mathrm{ft} \mathrm{E}$ of Col. $38 \mathrm{~W}$ \\
\hline VT299 & $39 \mathrm{~W}$ & $0.26 *$ & 0.26 & Floor & $9 \mathrm{ft} \mathrm{S} \& 14 \mathrm{ft} \mathrm{E}$ of Col. $39 \mathrm{~W}$ \\
\hline VT300 & $40 \mathrm{~W}$ & $0.28 *$ & 0.28 & Floor & $9 \mathrm{ft} \mathrm{S} \& 14 \mathrm{ft} E$ of Col $40 \mathrm{~W}$ \\
\hline VT301 & $41 \mathrm{~W}$ & $0.24 *$ & 0.24 & Floor & $9 \mathrm{ft} \mathrm{S} \& 14 \mathrm{ft} \mathrm{E}$ of Col. $41 \mathrm{~W}$ \\
\hline VT302 & $42 W$ & $0.18 *$ & 0.18 & Floor & $9 \mathrm{ft} \mathrm{S} \& 14 \mathrm{ft} \mathrm{E}$ of Col. $42 \mathrm{~W}$ \\
\hline VT303 & $43 W$ & $0.20 *$ & 0.20 & Floor & $9 \mathrm{ft} \mathrm{S} \& 14 \mathrm{ft} \mathrm{E}$ of Col. $43 \mathrm{~W}$ \\
\hline VT304 & NW bldg. & $0.19 *$ & 0.19 & Floor & $9 \mathrm{ft} \mathrm{S} \& 14 \mathrm{ft} E$ of $\mathrm{NW}$ corner of building \\
\hline VT305 & $43 W$ & $0.21 *$ & 0.21 & Floor & $\begin{array}{l}\text { Floor smear at } 10 \mathrm{ft} S \text { and } 4 \mathrm{ft} \& 5 \mathrm{in} . \mathrm{W} \text { of Col. } \\
43 \mathrm{~W}\end{array}$ \\
\hline VT306 & $42 W$ & $0.23 *$ & 0.23 & Floor & $\begin{array}{l}\text { Floor smear at } 10 \mathrm{ft} \mathrm{S} \text { and } 8 \mathrm{ft} \& 5 \mathrm{in} . \mathrm{W} \text { of Col. } \\
42 \mathrm{~W}\end{array}$ \\
\hline VT307 & $41 W$ & $0.22 *$ & 0.22 & Floor & Floor smear at $8 \mathrm{ft} S$ and $9 \mathrm{ft} \& 5 \mathrm{in} . \mathrm{W}$ of Col. $41 \mathrm{~W}$ \\
\hline VT308 & $40 \mathrm{~W}$ & $0.18 *$ & 0.18 & Floor & Floor smear at $10 \mathrm{ft} \& 5 \mathrm{in} . \mathrm{W} \& 2 \mathrm{ft} \mathrm{S}$ of Col. $40 \mathrm{~W}$ \\
\hline VT309 & $40 \mathrm{~W}$ & $0.19 *$ & 0.19 & Floor & $\begin{array}{l}\text { Floor smear at } 12 \mathrm{ft} S \text { and } 10 \mathrm{ft} \& 5 \mathrm{in} \text {. W of Col. } \\
40 \mathrm{~W}\end{array}$ \\
\hline VT310 & $39 \mathrm{~W}$ & $0.22 *$ & 0.22 & Floor & $\begin{array}{l}\text { Floor smear at } 9 \mathrm{ft} \mathrm{S} \text { and } 10 \mathrm{ft} \& 5 \mathrm{in} \text {. W of Col. } \\
39 \mathrm{~W}\end{array}$ \\
\hline VT311 & $38 \mathrm{~W}$ & $0.23 *$ & 0.23 & Floor & $\begin{array}{l}\text { Floor smear at } 9 \mathrm{ft} S \text { and } 10 \mathrm{ft} \& 5 \mathrm{in} . \mathrm{W} \text { of Col. } \\
38 \mathrm{~W}\end{array}$ \\
\hline VT312 & $37 \mathrm{~W}$ & $0.21 *$ & 0.21 & Floor & $\begin{array}{l}\text { Floor smear at } 9 \mathrm{ft} S \text { and } 10 \mathrm{ft} \& 5 \mathrm{in} \text {. W of Col. } \\
37 \mathrm{~W}\end{array}$ \\
\hline VT313 & $36 \mathrm{~W}$ & $0.20 *$ & 0.20 & Floor & Floor smear at $9 \mathrm{ft} \mathrm{S} \mathrm{\&} 10 \mathrm{ft} \mathrm{W}$ of Col. $36 \mathrm{~W}$ \\
\hline VT314 & $31-32 E$ & $0.21 *$ & 0.32 & Window & Window ledge between Col. $31 \mathrm{E} \& 32 \mathrm{E}$ \\
\hline
\end{tabular}


Table 4. (continued)

\begin{tabular}{|c|c|c|c|c|c|}
\hline $\begin{array}{l}\text { Sample } \\
\text { ID } a\end{array}$ & $\begin{array}{c}\text { Bay/room/ } \\
\text { structure/ } \\
\text { area }^{b}\end{array}$ & $\begin{array}{c}\mathrm{Be}^{c} \\
(\mu \mathrm{g} / \mathrm{g}) \\
(\mu \mathrm{g})^{*} \\
\end{array}$ & $\begin{array}{l}\text { Be/area } \\
\left(\mu \mathrm{g} / \mathrm{ft}^{2}\right)^{d}\end{array}$ & $\begin{array}{l}\text { Location } \\
\text { in bay }\end{array}$ & Location notese \\
\hline VT315 & $33-34 E$ & $0.19 *$ & 0.19 & Window & Window ledge between Col. 33E \& 34E \\
\hline VT316 & $35-36 \mathrm{E}$ & $0.22 *$ & 0.33 & Window & Window ledge between Col. $35 \mathrm{E} \& 36 \mathrm{E}$ \\
\hline VT317 & $37-38 \mathrm{E}$ & $0.29 *$ & 0.44 & Window & Window ledge between Col. 37E \& 38E \\
\hline VT318 & $39-40 E$ & $0.19 *$ & 0.29 & Window & Window ledge between Col. 39E \& $40 \mathrm{E}$ \\
\hline VT319 & $41-42 E$ & $0.13 *$ & 0.20 & Window & Window ledge between Col. $41 \mathrm{E} \& 42 \mathrm{E}$ \\
\hline VT320 & $31 \mathrm{~W}-32 \mathrm{~W}$ & $0.29 *$ & 0.58 & Window & Window ledge between Bay $31 \mathrm{~W} \& 32 \mathrm{~W}$ \\
\hline VT321 & $31 W-32 W$ & $0.12 *$ & 0.24 & Wall & Top of wall between Bay $31 \mathrm{~W} \& 32 \mathrm{~W}$ \\
\hline VT322 & $31 W-32 W$ & $0.22 *$ & 0.44 & Bridging & Top of bridging between Bay $31 W \& 32 W$ \\
\hline VT323 & $31 \mathrm{~W}$ & $0.40 *$ & 0.80 & Truss & Top of truss east of Col. $31 \mathrm{~W}$ \\
\hline VT324 & $32 W-33 W$ & $0.29 *$ & 0.58 & Window & Window ledge between Bay $32 W$ \& $33 W$ \\
\hline VT325 & $32 W-33 W$ & $0.18 *$ & 0.36 & Wall & Top of wall between Bay $32 \mathrm{~W} \& 33 \mathrm{~W}$ \\
\hline VT326 & $32 W-33 W$ & $0.19 *$ & 0.38 & Bridging & Top of bridging between Bay $32 W \& 33 W$ \\
\hline VT327 & $32 \mathrm{~W}$ & $0.20 *$ & 0.40 & Truss & $\begin{array}{l}\text { Top of lower horizontal member of truss about } 1 / 2 \\
\text { way to peak east of Col. } 32 \mathrm{~W}\end{array}$ \\
\hline VT328 & $33 \mathrm{~W}-34 \mathrm{~W}$ & $0.20 *$ & 0.40 & Window & Window ledge between Bay $33 W \& 34 W$ \\
\hline VT329 & $33 \mathrm{~W}-34 \mathrm{~W}$ & $0.26 *$ & 0.52 & Stringer & Stringer (visibly dirty) between Bay $33 \mathrm{~W} \& 34 \mathrm{~W}$ \\
\hline VT330 & $43 \mathrm{E}$ & $0.16 *$ & 0.16 & Window & Middle low window ledge between Col. 43E \& N wall \\
\hline VT331 & $31 \mathrm{E}$ & $0.31 *$ & 0.31 & Floor & $14 \mathrm{ft} \mathrm{W} \& 10 \mathrm{ft} \mathrm{S}$ of Col. $31 \mathrm{E}$ \\
\hline VT332 & $30 \mathrm{E}$ & $0.31 *$ & 0.31 & Floor & $14 \mathrm{ft} \mathrm{W} \& 10 \mathrm{ft} \mathrm{S}$ of Col. 30E \\
\hline VT333 & $29 \mathrm{E}$ & $0.21 *$ & 0.21 & Floor & $14 \mathrm{ft} \mathrm{W} \& 10 \mathrm{ft} \mathrm{S}$ of Col. $29 \mathrm{E}$ \\
\hline VT334 & $28 \mathrm{E}$ & $0.45 *$ & 0.45 & Floor & $14 \mathrm{ft} \mathrm{W} \mathrm{\&} 10 \mathrm{ft} \mathrm{S}$ of Col. 28E \\
\hline VT335 & $27 E$ & $0.45 *$ & 0.45 & Floor & $14 \mathrm{ft} \mathrm{W} \mathrm{\&} 10 \mathrm{ft} \mathrm{S}$ of Col. 27E \\
\hline VT336 & $26 \mathrm{E}$ & $0.31 *$ & 0.31 & Floor & $14 \mathrm{ft} \mathrm{W} \& 10 \mathrm{ft} \mathrm{S}$ of Col. $26 \mathrm{E}$ \\
\hline VT337 & $25 E$ & $0.95 *$ & 0.95 & Floor & $14 \mathrm{ft} \mathrm{W} \mathrm{\&} 10 \mathrm{ft} \mathrm{S}$ of Col. $25 \mathrm{E}$ \\
\hline VT338 & $24 \mathrm{E}$ & $0.37 *$ & 0.37 & Floor & $14 \mathrm{ft} \mathrm{W} \& 10 \mathrm{ft} \mathrm{S}$ of Col. 24E \\
\hline VT339 & $23 E$ & $0.44 *$ & 0.44 & Floor & $14 \mathrm{ft} \mathrm{W} \mathrm{\&} 10 \mathrm{ft} \mathrm{S}$ of Col. 23E \\
\hline VT340 & $22 \mathrm{E}$ & $0.23 *$ & 0.23 & Floor & $14 \mathrm{ft} \mathrm{W} \& 10 \mathrm{ft} \mathrm{S}$ of Col. 22E \\
\hline VT341 & $22 \mathrm{~W}$ & $0.30 *$ & 0.30 & Floor & $34 \mathrm{ft} \mathrm{E} \mathrm{\&} 10 \mathrm{ft} \mathrm{S}$ of Col. $22 \mathrm{~W}$ \\
\hline VT342 & $22 \mathrm{~W}$ & 0.20 * & 0.20 & Floor & $14 \mathrm{ftE} \& 10 \mathrm{ft} S$ of Col. $22 \mathrm{~W}$ \\
\hline VT343 & $23 \mathrm{~W}$ & $0.25 *$ & 0.25 & Floor & $14 \mathrm{ft} \mathrm{E} \mathrm{\&} 9 \mathrm{ft} \mathrm{S}$ of Col. $23 \mathrm{~W}$ \\
\hline VT344 & $23 \mathrm{~W}$ & $0.23 *$ & 0.23 & Floor & $34 \mathrm{ft} \mathrm{E} \mathrm{\&} 10 \mathrm{ft} \mathrm{S}$ of Col. 23W \\
\hline VT345 & $24 \mathrm{~W}$ & $0.40 *$ & 0.40 & Floor & $14 \mathrm{ft} E \& 10 \mathrm{ft} \mathrm{S}$ of Col. $24 \mathrm{~W}$ \\
\hline
\end{tabular}


Table 4. (continued)

\begin{tabular}{|c|c|c|c|c|c|}
\hline $\begin{array}{l}\text { Sample } \\
\text { ID } a\end{array}$ & $\begin{array}{c}\text { Bay/room/ } \\
\text { structure/ } \\
\text { area }^{b}\end{array}$ & $\begin{array}{c}\mathrm{Be}^{c} \\
(\mu \mathrm{g} / \mathrm{g}) \\
(\mu \mathrm{g})^{*} \\
\end{array}$ & $\begin{array}{c}\text { Be/area } \\
\left(\mu \mathrm{g} / \mathrm{ft}^{2}\right)^{d}\end{array}$ & $\begin{array}{l}\text { Location } \\
\text { in bay }\end{array}$ & Location notese \\
\hline VT346 & $25 \mathrm{~W}$ & $1.2^{*}$ & 1.2 & Floor & $14 \mathrm{ft} \mathrm{E} \mathrm{\&} 9 \mathrm{ft} \mathrm{S}$ of Col. $25 \mathrm{~W}$ \\
\hline VT347 & $26 \mathrm{~W}$ & $0.14 *$ & 0.14 & Floor & $14 \mathrm{ft} E \& 10 \mathrm{ft} \mathrm{S}$ of Col. $26 \mathrm{~W}$ \\
\hline VT348 & $27 \mathrm{~W}$ & $0.11 *$ & 0.11 & Floor & $14 \mathrm{ft} E \& 10 \mathrm{ft} \mathrm{S}$ of $\mathrm{Col} 27 \mathrm{~W}$ \\
\hline VT349 & $28 W$ & $0.22 *$ & 0.22 & Floor & $14 \mathrm{ft} \mathrm{E} \mathrm{\&} 10 \mathrm{ft} \mathrm{S}$ of Col. $28 \mathrm{~W}$ \\
\hline VT350 & $29 \mathrm{~W}$ & $0.22 *$ & 0.22 & Floor & $14 \mathrm{ft} E \& 10 \mathrm{ft} \mathrm{S}$ of Col. $29 \mathrm{~W}$ \\
\hline VT351 & $30 \mathrm{~W}$ & $0.13 *$ & 0.13 & Floor & $14 \mathrm{ft} E \& 10 \mathrm{ft} \mathrm{S}$ of Col. $30 \mathrm{~W}$ \\
\hline VT352 & $31 \mathrm{~W}$ & $0.17 *$ & 0.17 & Floor & $14 \mathrm{ft} \mathrm{E} \mathrm{\&} 10 \mathrm{ft} \mathrm{S}$ of Col. $31 \mathrm{~W}$ \\
\hline VT353 & 29-30E & $0.19 *$ & 0.29 & Window & Window ledge between Col. $29 \mathrm{E} \& 30 \mathrm{E}$ \\
\hline VT354 & $27-28 \mathrm{E}$ & $0.28 *$ & 0.42 & Window & Window ledge between Col. 27E \& 28E \\
\hline VT355 & $25-26 \mathrm{E}$ & $0.14 *$ & 0.21 & Window & Window ledge between Col. $25 \mathrm{E} \& 26 \mathrm{E}$ \\
\hline VT356 & $25-24 \mathrm{E}$ & $0.69 *$ & 0.69 & Window & Window ledge between Col. 25E \& 24E \\
\hline VT357 & MS-1 & $0.30 *$ & 0.30 & Floor & $9 \mathrm{ft} W \& 9 \mathrm{ft} S$ of Col. $25 \mathrm{~W}$ in Rm. MS- 1 \\
\hline VT358 & MS-1 & $0.66 *$ & 0.66 & Floor & $7 \mathrm{ft} \mathrm{S} \& 8 \mathrm{ft} \mathrm{E}$ of Col. $24 \mathrm{~W}$ in Rm. MS-1 \\
\hline VT359 & $28-29 \mathrm{~W}$ & $0.19 *$ & 0.19 & Floor & $\begin{array}{l}\text { Floor in men's room located between Col. } 28 \mathrm{~W} \& \\
29 \mathrm{~W}\end{array}$ \\
\hline VT360 & $33 W-34 W$ & $0.40 *$ & 0.80 & Bridging & Bridging between Bay $33 \mathrm{~W} \& 34 \mathrm{~W}$ \\
\hline VT361 & $33 W$ & $0.18 *$ & 0.36 & Truss & Top of truss east of Col. $33 \mathrm{~W}$ \\
\hline VT362 & $34 \mathrm{~W}$ & $0.40^{*}$ & 0.80 & Truss & Top of lower truss near Col. $34 \mathrm{~W}$ \\
\hline VT363 & $34 W-35 W$ & $0.46^{*}$ & 0.92 & Other & Top of cross brace at truss of $34 \mathrm{~W} \& 35 \mathrm{~W}$ \\
\hline VT364 & $35 \mathrm{~W}$ & $0.58 *$ & 1.2 & Truss & Top of truss E from Col. $35 \mathrm{~W}$ \\
\hline VT365 & $35 W-36 W$ & $0.50 *$ & 1.0 & Bridging & Top of bridging between Bay $35 \mathrm{~W} \& 36 \mathrm{~W}$ \\
\hline VT366 & $36 \mathrm{~W}$ & $0.21 *$ & 0.42 & Truss & Top of truss east from Col $36 \mathrm{~W}$ \\
\hline VT367 & $36 \mathrm{~W}-37 \mathrm{~W}$ & $0.21 *$ & 0.42 & Bridging & Top of bridging between Bay $36 \mathrm{~W} \& 37 \mathrm{~W}$ \\
\hline VT368 & $36 \mathrm{~W}-37 \mathrm{~W}$ & $0.25 *$ & 0.50 & Stringer & Upper side of stringer between $36 \mathrm{~W} \& 37 \mathrm{~W}$ \\
\hline VT370 & $37 \mathrm{~W}-38 \mathrm{~W}$ & $0.25 *$ & 0.50 & Stringer & Dirty stringer between Col. $37 \mathrm{~W} \& 38 \mathrm{~W}$ \\
\hline VT371 & MS-6 & $0.66 *$ & 0.66 & Floor & $5 \mathrm{ft} \mathrm{N} \& 9 \mathrm{ft} E$ of Col. 25E in Rm. MS-6 \\
\hline VT372 & MS-6 & $0.44 *$ & 0.44 & Window & Window ledge (E.side) in Rm. MS-6 \\
\hline VT373 & MS-6 & $0.37 *$ & 0.37 & Floor & Room north of vault and near Col. $25 \mathrm{E}$ \\
\hline VT374 & Vault & $0.58 *$ & 0.58 & Floor & $9 \mathrm{ft} \mathrm{S} \mathrm{\&} 7 \mathrm{ft} \mathrm{E}$ of Col. $24 \mathrm{E}$ inside the vault \\
\hline VT377 & $31-32 E$ & $0.12 *$ & 0.24 & Wall & Top of plate between Col. $31 \mathrm{E} \& 32$ \\
\hline VT378 & $32-33 E$ & $0.27 *$ & 0.27 & Window & Ledge of window between Col. $32 \mathrm{E} \& 33 \mathrm{E}$ \\
\hline VT379 & $32-33 E$ & $0.17 *$ & 0.34 & Wall & Top of plate between Col. 32E \& 33E \\
\hline
\end{tabular}


Table 4. (continued)

\begin{tabular}{|c|c|c|c|c|c|}
\hline $\begin{array}{c}\text { Sample } \\
\text { ID } a\end{array}$ & $\begin{array}{c}\text { Bay/room/ } \\
\text { structure/ } \\
\text { area }^{b}\end{array}$ & $\begin{array}{c}\mathrm{Be}^{c} \\
(\mu \mathrm{g} / \mathrm{g}) \\
(\mu \mathrm{g})^{*} \\
\end{array}$ & $\begin{array}{c}\mathrm{Be} / \mathrm{area} \\
\left(\mu \mathrm{g} / \mathrm{ft}^{2}\right)^{d} \\
\end{array}$ & $\begin{array}{l}\text { Location } \\
\text { in bay }\end{array}$ & Location notes ${ }^{e}$ \\
\hline VT380 & 33-34E & $0.18 *$ & 0.46 & Wall & Top of plate between Col. 33E \& 34E \\
\hline VT381 & $38 \mathrm{~W}$ & $0.41 *$ & 0.82 & Truss & Top of truss east of Col. line $38 \mathrm{~W}$ \\
\hline VT382 & $38-39 W$ & $0.19 *$ & 0.38 & Bridging & Top of bridging between Col. $38 \mathrm{~W} \& 39 \mathrm{~W}$ \\
\hline VT383 & $40-41 W$ & $0.19 *$ & 0.38 & Bridging & Top of bridging east \& between $40 \mathrm{~W} \& 41 \mathrm{~W}$ \\
\hline VT384 & $41 \mathrm{~W}$ & $0.13 *$ & 0.26 & Truss & Top of truss east of Col. line $41 \mathrm{~W}$ \\
\hline VT385 & $41 W-42 W$ & $0.16 *$ & 0.32 & Bridging & Top of bridging between $41 \mathrm{~W} \& 42 \mathrm{~W}$ \\
\hline VT386 & $41 W-42 W$ & $0.16 *$ & 0.32 & Wall & Top of wall between Col. $41 \mathrm{~W} \& 42 \mathrm{~W}$ \\
\hline VT387 & $41 W-42 W$ & $0.13^{*}$ & 0.26 & Window & Window ledge between Col. $41 \mathrm{~W} \& 42 \mathrm{~W}$ \\
\hline VT388 & $42 \mathrm{~W}$ & $0.15 *$ & 0.30 & Truss & Top of truss east of Col. $42 \mathrm{~W}$ \\
\hline VT389 & $42 W-43 W$ & $0.10 *$ & 0.20 & Bridging & Top of bridging east \& between $42 \mathrm{~W} \& 43 \mathrm{~W}$ \\
\hline VT390 & $42 W-43 W$ & $0.22 *$ & 0.44 & Wall & Top of wall between Col. $42 \mathrm{~W} \& 43 \mathrm{~W}$ \\
\hline VT391 & $42 W-43 W$ & $0.19 *$ & 0.38 & Window & Window ledge between Col. $42 \mathrm{~W} \& 43 \mathrm{~W}$ \\
\hline VT392 & $43 \mathrm{~W}$ & $0.10 *$ & 0.20 & Window & Window ledge between Col. 43 W \& NW bldg. \\
\hline VT393 & $44 \mathrm{E}-44 \mathrm{~W}$ & $0.18 *$ & 0.36 & Wall & Top of wall on far north end of bldg. \\
\hline VT394 & $43-44 E$ & $0.22 *$ & 0.44 & Truss & $\begin{array}{l}\text { Top of lower west side truss brace between Truss } 43 \\
\& \text { north end of building }\end{array}$ \\
\hline VT395 & $29 \mathrm{~W}$ & $0.10^{*}$ & 0.20 & Other & $\begin{array}{l}\text { Upper horizontal surface of steam line at } 12 \mathrm{ft} \mathrm{S} \mathrm{\&} 13 \\
\mathrm{ft} \text { W of Col. } 29 \mathrm{~W}\end{array}$ \\
\hline VT396 & $29 \mathrm{~W}$ & $0.68 *$ & 1.4 & Other & $\begin{array}{l}\text { Surface (visibly dirty) of sprinkler line at } 17 \mathrm{ft} N \& 6 \\
\mathrm{ft} W \text { of Col. } 29 \mathrm{~W}\end{array}$ \\
\hline VT397 & $29 \mathrm{~W}$ & $0.12 *$ & 0.24 & Other & Visibly dirty wooden cross brace near Col. $29 \mathrm{~W}$ \\
\hline VT398 & $29 \mathrm{~W}$ & $0.12 *$ & 0.24 & Bridging & Visibly dirty bridging located near Col. $29 \mathrm{~W}$ \\
\hline VT399 & $34-35 W$ & $0.21 *$ & 0.42 & Window & Window ledge between Col. $34 \mathrm{~W} \& 35 \mathrm{~W}$ \\
\hline VT400 & $34-35 W$ & $0.12 *$ & 0.24 & Stringer & Vertical board between Col. $34 \mathrm{~W} \& 35 \mathrm{~W}$ \\
\hline VT401 & $34-35 E$ & $0.22 *$ & 0.44 & Wall & Top of plate between Col. 34E \& 35E \\
\hline VT402 & $34-35 E$ & $0.25 *$ & 0.25 & $\begin{array}{l}\text { Window } \\
\text { ledge }\end{array}$ & Ledge of window between Col. 34E \& 35E \\
\hline VT403 & $35-36 E$ & $0.17 *$ & 0.34 & Wall & Top of plate between Col. 35E \& 36E \\
\hline VT404 & $36-37 E$ & $0.20 *$ & 0.40 & Wall & Top of plate between Col. 36E \& 37E \\
\hline VT405 & $36-37 E$ & $0.37 *$ & 0.37 & Window & Window ledge between Col. 36E \& 37E \\
\hline VT406 & $37-38 \mathrm{E}$ & $0.16 *$ & 0.32 & Wall & Top of plate between Col. $37 \mathrm{E} \& 38 \mathrm{E}$ \\
\hline VT407 & $38-39 \mathrm{E}$ & $0.15 *$ & 0.30 & Wall & Top of plate between Col. 38E \& 39E \\
\hline VT408 & $38-39 E$ & $0.43 *$ & 0.43 & Window & Upper window ledge between Col. 38E \& 39E \\
\hline
\end{tabular}


Table 4. (continued)

\begin{tabular}{|c|c|c|c|c|c|}
\hline $\begin{array}{c}\text { Sample } \\
\text { ID }^{a} \\
\end{array}$ & $\begin{array}{c}\text { Bay/room/ } \\
\text { structure/ } \\
\text { area }^{b}\end{array}$ & $\begin{array}{c}\mathrm{Be}^{c} \\
(\mu \mathrm{g} / \mathrm{g}) \\
(\mu \mathrm{g})^{*} \\
\end{array}$ & $\begin{array}{c}\text { Be/area } \\
\left(\mu \mathrm{g} / \mathrm{ft}^{2}\right)^{d} \\
\end{array}$ & $\begin{array}{l}\text { Location } \\
\text { in bay }\end{array}$ & Location notese \\
\hline VT409 & $39-40 \mathrm{E}$ & $0.28 *$ & 0.56 & Wall & Top of plate between Col. 39E \& 40E \\
\hline VT410 & $40-41 E$ & $0.23 *$ & 0.46 & Wall & Top of plate between Col. $40 \mathrm{E} \& 41 \mathrm{E}$ \\
\hline VT411 & $40-41 E$ & $0.44 *$ & 0.44 & Window & Upper window ledge between Col. $40 \mathrm{E} \& 41 \mathrm{E}$ \\
\hline VT412 & $41-42 \mathrm{E}$ & $0.16 *$ & 0.32 & Wall & Top of plate between Col. 41E \& 42E \\
\hline VT413 & $42-43 E$ & $0.13 *$ & 0.26 & Wall & Top of plate between Col. $42 \mathrm{E} \& 43 \mathrm{E}$ \\
\hline VT4 14 & $42-43 E$ & $0.26 *$ & 0.26 & Window & Upper window between Col. 42E \& 43E \\
\hline VT415 & $43 \mathrm{E}$ & $0.17 *$ & 0.17 & Wall & Top of plate between Col. $43 \mathrm{E} \&$ end of building \\
\hline VT416 & $33-34 W$ & $0.21 *$ & 0.42 & Stringer & Vertical board between Col. $33 \mathrm{~W} \& 34 \mathrm{~W}$ \\
\hline VT417 & $35 \mathrm{~W}$ & $0.20 *$ & 0.20 & Other & Top of heater near Col. $35 \mathrm{~W}$ \\
\hline VT418 & $35-36 \mathrm{~W}$ & $0.19 *$ & 0.38 & Window & Window ledge between Col. $35 \mathrm{~W} \& 36 \mathrm{~W}$ \\
\hline VT419 & $35-36 W$ & $0.13 *$ & 0.26 & Wall & Top of block wall between Col. $35 \mathrm{~W} \& 36 \mathrm{~W}$ \\
\hline VT420 & $36-37 \mathrm{~W}$ & $0.22 *$ & 0.44 & Window & Window ledge between Col. 36W \& 37W \\
\hline VT421 & $36-37 \mathrm{~W}$ & $0.13 *$ & 0.26 & Stringer & Vertical board between Col. $36 \mathrm{~W} \& 37 \mathrm{~W}$ \\
\hline VT422 & $37-38 \mathrm{~W}$ & $0.18 *$ & 0.36 & Window & Window ledge between Col. $37 \mathrm{~W} \& 38 \mathrm{~W}$ \\
\hline VT423 & $43 W$ & $0.10 *$ & 0.20 & Truss & Top of truss near Col. $43 \mathrm{~W}$ \\
\hline VT424 & $43 \mathrm{~W}$ & $0.10^{*}$ & 0.20 & Wall & Truss plate between Col. $43 \mathrm{~W} \& \mathrm{NW}$ corner \\
\hline VT425 & $43 \mathrm{~W}$ & $0.12 *$ & 0.24 & Bridging & Bridging between Col. $43 \mathrm{~W} \& \mathrm{NW}$ corner \\
\hline VT426 & $40-41 W$ & $0.17 *$ & 0.34 & Window & Window ledge between Col. $40 \mathrm{~W} \& 41 \mathrm{~W}$ \\
\hline VT427 & $40-41 W$ & $0.11 *$ & 0.22 & Wall & Top of block wall between Col. $40 \mathrm{~W} \& 41 \mathrm{~W}$ \\
\hline VT428 & $37-38 W$ & $0.10 *$ & 0.20 & Wall & Top of block wall between Col. $37 \mathrm{~W} \& 38 \mathrm{~W}$ \\
\hline VT429 & $37 \mathrm{~W}$ & $0.10 *$ & 0.20 & Truss & Top of truss from Col. $37 \mathrm{~W}$ \\
\hline VT430 & $37-38 W$ & $0.10 *$ & 0.20 & Stringer & Stringer located between Col. $37 \mathrm{~W} \& 38 \mathrm{~W}$ \\
\hline VT431 & $37-38 \mathrm{~W}$ & $0.15^{*}$ & 0.30 & Bridging & Bridging between Col. 37W \& 38W \\
\hline VT432 & $38-39 \mathrm{~W}$ & $0.19 *$ & 0.38 & Window & Window ledge between Col. $38 \mathrm{~W} \& 39 \mathrm{~W}$ \\
\hline VT433 & $38-39 W$ & $0.17 *$ & 0.34 & Wall & Top of block wall between Col. $38 \mathrm{~W} \& 39 \mathrm{~W}$ \\
\hline VT434 & $31-32 E$ & $0.23 *$ & 0.46 & Bridging & Bridging between Col. 31E \& 32E \\
\hline VT435 & $31 \mathrm{E}$ & $0.22 *$ & 0.44 & Truss & Top of lower truss brace near Col. 31E \\
\hline VT436 & $32-33 E$ & $0.19 *$ & 0.38 & Bridging & Bridging between Col. $32 \mathrm{E}^{-} \& 33 \mathrm{E}$ \\
\hline VT437 & $32 \mathrm{E}$ & $0.19 *$ & 0.38 & Truss & Top of truss about 12 way to the peak from Col. $32 \mathrm{E}$ \\
\hline VT438 & $33 \mathrm{E}$ & $0.15 *$ & 0.30 & Truss & Top of truss about 12 way to the peak from Col. $33 \mathrm{E}$ \\
\hline VT439 & $33-34 \mathrm{E}$ & $0.11 *$ & 0.22 & Bridging & Bridging between Col. 33E \& 34E \\
\hline
\end{tabular}


Table 4. (continued)

\begin{tabular}{|c|c|c|c|c|c|}
\hline $\begin{array}{l}\text { Sample } \\
\text { ID } a\end{array}$ & $\begin{array}{c}\text { Bay/room/ } \\
\text { structure/ } \\
\text { areab }\end{array}$ & $\begin{array}{c}\mathrm{Be}^{c} \\
(\mu \mathrm{g} / \mathrm{g}) \\
(\mu \mathrm{g})^{*}\end{array}$ & $\begin{array}{c}\text { Be/area } \\
\left(\mu \mathrm{g} / \mathrm{ft}^{2}\right)^{d} \\
\end{array}$ & $\begin{array}{l}\text { Location } \\
\text { in bay }\end{array}$ & Location notese \\
\hline VT440 & $34-35 E$ & $0.14 *$ & 0.28 & Bridging & Bridging between Col. 34E \& 35E \\
\hline VT441 & $34 \mathrm{E}$ & $0.12 *$ & 0.24 & Truss & Top of truss near Col. 34E \\
\hline VT442 & $35 \mathrm{E}$ & $0.10 *$ & 0.20 & Truss & Top of truss near Col. 35E \\
\hline VT443 & $35-36 \mathrm{E}$ & $0.29 *$ & 0.58 & Bridging & Bridging (dusty) between Col. 35E \& 36E \\
\hline VT444 & $36 \mathrm{E}$ & $0.10 *$ & 0.20 & Truss & Top of truss near Col. 36E \\
\hline VT445 & $36-37 E$ & $0.10 *$ & 0.20 & Bridging & Bridging between Col. 36E \& 37E \\
\hline VT446 & $37 \mathrm{E}$ & $0.10 *$ & 0.20 & Truss & Top of truss near Col. 37E \\
\hline VT447 & $37-38 E$ & $0.15^{*}$ & 0.30 & Bridging & Bridging between Col. 37E \& 38E \\
\hline VT448 & $38 \mathrm{E}$ & $0.10 *$ & 0.20 & Truss & Top of truss brace near Col. $38 \mathrm{E}$ \\
\hline VT449 & $38-39 E$ & $0.10 *$ & 0.20 & Stringer & Stringer between Col. 38E \& 39E \\
\hline VT450 & $38-39 E$ & $0.10 *$ & 0.20 & Bridging & Bridging between Col. 38E \& 39E \\
\hline VT451 & $30-31 E$ & $0.26 *$ & 0.52 & Wall & Top of plate between Col. 30E \& 31E \\
\hline VT452 & $30-31 \mathrm{E}$ & $0.19 *$ & 0.19 & $\begin{array}{l}\text { Window } \\
\text { ledge }\end{array}$ & $\begin{array}{l}\text { Window (upper middle) ledge (sm. amt. of debris in } \\
\text { corner) between Col. } 30 \mathrm{E} \& 31 \mathrm{E}\end{array}$ \\
\hline VT453 & $29-30 \mathrm{E}$ & $0.38 *$ & 0.76 & Wall & Top of plate between Col. 29E \& 30E \\
\hline VT454 & $28-29 \mathrm{E}$ & $0.34 *$ & 0.68 & Wall & Top of plate between Col. $28 \mathrm{E} \& 29 \mathrm{E}$ \\
\hline VT456 & $27-28 E$ & $0.81 *$ & 1.6 & Wall & Top of plate between Col. $27 \mathrm{E} \& 28 \mathrm{E}$ \\
\hline VT457 & $26-27 \mathrm{E}$ & $0.18 *$ & 0.36 & Wall & Top of plate between Col. 26E \& $27 \mathrm{E}$ \\
\hline VT460 & $25-26 \mathrm{E}$ & $0.74 *$ & 1.5 & Wall & Top of plate between Col. 25E \& 26E \\
\hline VT461 & $21-22 \mathrm{E}$ & $0.44 *$ & 0.44 & Wall & Top of plate (visibly dirty) between Col. $21 \mathrm{E} \& 22 \mathrm{E}$ \\
\hline VT462 & $21-22 E$ & $0.20 *$ & 0.20 & Other & Top of header between Col. $21 \mathrm{E} \& 22 \mathrm{E}$ \\
\hline VT463 & $22-23 E$ & $0.31 *$ & 0.31 & Other & Top of header between Col. 22E \& 23E \\
\hline VT464 & 22-23E & $0.80 *$ & 0.80 & Wall & Top of plate (visibly dirty) between Col. 22E \& 23E \\
\hline VT465 & $23-24 \mathrm{E}$ & $0.81 *$ & 1.6 & Wall & Top of plate between Col.23E \& 24E \\
\hline VT467 & MS-1 & $0.19 *$ & 0.19 & Wall & $\begin{array}{l}\text { Top of wall in MS-1 (furnace room) about } 11 \mathrm{ft} 9 \mathrm{in} \text {. } \\
\text { off the floor on southside }\end{array}$ \\
\hline VT468 & $23-24 W$ & $0.57 *$ & 0.57 & Other & Top of the lower header between Col: $23 \mathrm{~W} \& 24 \mathrm{~W}$ \\
\hline VT469 & $23-24 W$ & $0.18 *$ & 0.36 & Wall & $\begin{array}{l}\text { Top of plate (visibly very dirty) between Col. } 23 W \& \\
24 W\end{array}$ \\
\hline VT470 & $23 W$ & $0.22 *$ & 0.22 & Truss & Top of beam at Col. $23 \mathrm{~W}$ \\
\hline VT471 & $28-29 W$ & $0.37 *$ & 0.37 & Window & Upper center window ledge between Col. $28 \mathrm{~W} \& 29 \mathrm{~W}$ \\
\hline VT472 & $28-29 \mathrm{~W}$ & $0.20 *$ & 0.40 & Wall & Top of plate between Col. $28 \mathrm{~W} \& 29 \mathrm{~W}$ \\
\hline
\end{tabular}


Table 4. (continued)

\begin{tabular}{|c|c|c|c|c|c|}
\hline $\begin{array}{c}\text { Sample } \\
\text { ID } a\end{array}$ & $\begin{array}{c}\text { Bay/room/ } \\
\text { structure/ } \\
\text { area }^{b}\end{array}$ & $\begin{array}{c}\mathrm{Be}^{\mathrm{c}} \\
(\mu \mathrm{g} / \mathrm{g}) \\
(\mu \mathrm{g})^{*}\end{array}$ & $\begin{array}{c}\text { Be/area } \\
\left(\mu \mathrm{g} / \mathrm{ft}^{2}\right)^{d}\end{array}$ & $\begin{array}{l}\text { Location } \\
\text { in bay }\end{array}$ & Location notese \\
\hline VT473 & $27-28 W$ & $0.18 *$ & 0.18 & Window & Upper center window ledge between Col. $27 \mathrm{~W} \& 28 \mathrm{~W}$ \\
\hline VT474 & $27-28 W$ & $0.20 *$ & 0.40 & Wall & Top of plate between Col. $27 \mathrm{~W} \& 28 \mathrm{~W}$ \\
\hline VT475 & $26-27 \mathrm{~W}$ & $0.36 *$ & 0.36 & Window & Upper center window ledge between Col. $26 \mathrm{~W} \& 27 \mathrm{~W}$ \\
\hline VT476 & $26-27 \mathrm{~W}$ & $0.18 *$ & 0.36 & Wall & Top of plate between Col. $26 \mathrm{~W} \& 27 \mathrm{~W}$ \\
\hline VT477 & $29-30 \mathrm{~W}$ & $0.46 *$ & 0.46 & Window & Upper south window ledge between Col. $29 \mathrm{~W} \& 30 \mathrm{~W}$ \\
\hline VT478 & $29-30 \mathrm{~W}$ & $0.18 *$ & 0.36 & Wall & Top of plate between Col. $29 \mathrm{~W} \& 30 \mathrm{~W}$ \\
\hline VT479 & $30-31 W$ & $0.32 *$ & 0.32 & Window & $\begin{array}{l}\text { Upper middle window ledge between Col. } 30 \mathrm{~W} \& \\
31 \mathrm{~W}\end{array}$ \\
\hline VT480 & $39-40 W$ & $0.14 *$ & 0.28 & Window & $\begin{array}{l}\text { Center concrete window ledge between Col. } 39 \mathrm{~W} \\
\& 40 \mathrm{~W}\end{array}$ \\
\hline VT481 & $39-40 \mathrm{~W}$ & $0.10 *$ & 0.20 & Wall & Top of concrete block wall between Col. $39 \mathrm{~W} \& 40 \mathrm{~W}$ \\
\hline VT482 & $40 \mathrm{~W}$ & $0.10 *$ & 0.20 & Truss & Top of truss about 12 way to peak from Col. $40 \mathrm{~W}$ \\
\hline VT483 & $40 \mathrm{E}$ & $0.10 *$ & 0.20 & Truss & Top of truss about 12 way to peak from Col. $40 \mathrm{E}$ \\
\hline VT484 & $40-41 E$ & $0.10 *$ & 0.20 & Bridging & $\begin{array}{l}\text { Bridging about } 12 \text { way to peak from wall between } \\
\text { Col } 40 \mathrm{E} \& 41 \mathrm{E}\end{array}$ \\
\hline VT485 & $41-42 E$ & $0.45 *$ & 0.90 & Bridging & $\begin{array}{l}\text { Bridging about } 12 \text { way to peak from wall between } \\
\text { Col. } 41 E \& 42 E\end{array}$ \\
\hline VT486 & $41 E$ & $0.23 *$ & 0.46 & Truss & $\begin{array}{l}\text { Top of lower horizontal truss member about } 12 \text { way } \\
\text { to peak from Col. } 41 \mathrm{E}\end{array}$ \\
\hline VT487 & $42 \mathrm{E}$ & $0.14 *$ & 0.28 & Truss & Top of truss about 12 way to peak from Col. $42 \mathrm{E}$ \\
\hline VT488 & $42-43 E$ & $0.17 *$ & 0.34 & Bridging & $\begin{array}{l}\text { Bridging about } 12 \text { way to the peak from the wall } \\
\text { between Col. } 42 E \& 43 E\end{array}$ \\
\hline VT489 & $43 \mathrm{E}$ & $0.13 *$ & 0.26 & Truss & Top of truss about 12 way to the peak from Col. $43 \mathrm{E}$ \\
\hline VT490 & $42-43 E$ & $0.19 *$ & 0.38 & Stringer & $\begin{array}{l}\text { Upper surface of stringer about } 12 \text { way to peak from } \\
\text { wall between Col. } 42 E \text { \& } 43 E\end{array}$ \\
\hline VT491 & $42-43 E$ & $0.20 *$ & 0.40 & Bridging & Bridging between Col. 42E \& 43E \\
\hline VT492 & NE bldg. & $0.25^{*}$ & 0.50 & Wall & Top of wood plate in NE comer of bldg. \\
\hline VT493 & $43 \mathrm{E}$ & $0.56 *$ & 1.1 & Bridging & Bridging between Col. 43E \& NE corner of building \\
\hline VT494 & $43 \mathrm{E}$ & $0.21 *$ & 0.42 & Truss & $\begin{array}{l}\text { Top of truss about } 1 \mathrm{ft} \text { east of roof peak on Col. line } \\
43 \mathrm{E}-\mathrm{W}\end{array}$ \\
\hline VT495 & $39 \mathrm{E}$ & $0.20 *$ & 0.40 & Truss & Top of truss about 12 way to peak from Col. $39 \mathrm{E}$ \\
\hline VT496 & $39-40 \mathrm{E}$ & $0.24 *$ & 0.48 & Bridging & $\begin{array}{l}\text { Bridging about } 12 \text { way to peak from wall between } \\
\text { Col. } 39 \mathrm{E} \& 40 \mathrm{E}\end{array}$ \\
\hline VT497 & $39-40 \mathrm{~W}$ & $0.17 *$ & 0.34 & Bridging & Bridging between Col. $39 \mathrm{~W} \& 40 \mathrm{~W}$ \\
\hline
\end{tabular}


Table 4. (continued)

\begin{tabular}{|c|c|c|c|c|c|}
\hline $\begin{array}{c}\text { Sample } \\
\text { IDe }\end{array}$ & $\begin{array}{c}\text { Bay/room/ } \\
\text { structure/ } \\
\text { area }^{b}\end{array}$ & $\begin{array}{c}\mathrm{Be}^{c} \\
(\mu \mathrm{g} / \mathrm{g}) \\
(\mu \mathrm{g})^{*}\end{array}$ & $\begin{array}{c}\text { Be/area } \\
\left(\mu \mathrm{g} / \mathrm{ft}^{2}\right)^{d}\end{array}$ & $\begin{array}{l}\text { Location } \\
\text { in bay }\end{array}$ & Location notese \\
\hline VT498 & $39 \mathrm{~W}$ & $0.17 *$ & 0.34 & Truss & Top of truss near Col. $39 \mathrm{~W}$ \\
\hline VT499 & $39-40 \mathrm{~W}$ & $0.17 *$ & 0.34 & Stringer & Stringer between Col. $39 \mathrm{~W} \& 40 \mathrm{~W}$ \\
\hline VT500 & $30-31 \mathrm{E}$ & $0.15 *$ & 0.30 & Bridging & $\begin{array}{l}\text { Bridging about } 12 \text { way to peak from wall between } \\
\text { Col. } 30 \mathrm{E} \& 31 \mathrm{E}\end{array}$ \\
\hline VT501 & $30-31 \mathrm{~W}$ & $0.10 *$ & 0.20 & Wall & Top of plate between Col. $30 \mathrm{~W} \& 31 \mathrm{~W}$ \\
\hline VT502 & $25-26 \mathrm{~W}$ & $0.98 *$ & 0.98 & Window & Upper north window ledge between Col. $25 \mathrm{~W} \& 26 \mathrm{~W}$ \\
\hline VT503 & $25-26 \mathrm{~W}$ & $0.10 *$ & 0.20 & Wall & Top of plate between Col. $25 \mathrm{~W} \& 26 \mathrm{~W}$ \\
\hline VT504 & $26 \mathrm{E}$ & $0.31 *$ & 0.62 & Truss & Top of lower truss Col. 26 middle (little bit east) \\
\hline VT505 & $27 \mathrm{E}$ & $0.88 *$ & 1.8 & Truss & $\begin{array}{l}\text { Top of lower truss between (east of center so nearer to } \\
\text { E Col.) Col. } 27 \mathrm{E} \& 27 \mathrm{~W}\end{array}$ \\
\hline VT506 & $27 \mathrm{E}$ & $0.20 *$ & 0.40 & Truss & $\begin{array}{l}\text { Top of upper truss between (east of center so nearer to } \\
\text { Col. E) Col. } 27 \mathrm{E} \mathrm{\&} 27 \mathrm{~W}\end{array}$ \\
\hline VT507 & $27-28 E$ & $0.33 *$ & 0.66 & Bridging & $\begin{array}{l}\text { Bridging between (east of center so nearer E Cols.) } \\
\text { Col. } 27 \& 28\end{array}$ \\
\hline VT509 & $27-28 \mathrm{E}$ & $0.20 *$ & 0.40 & Stringer & Stringer between $27 \mathrm{E} \& 28 \mathrm{E}$ east of center at Col. 28 \\
\hline VT512 & $24-25 E$ & $1.3^{*}$ & 1.3 & Window & Upper south window ledge between Col. 24E \& $25 E$ \\
\hline VT513 & $22-23 W$ & $0.10 *$ & 0.10 & Wall & $\begin{array}{l}\text { Top of "I" beam above garage door between } 22 \mathrm{~W} \\
\& 23 \mathrm{~W} \text {. Equivalent to top of plate. }\end{array}$ \\
\hline VT514 & $21-22 \mathrm{~W}$ & $0.10 *$ & 0.10 & Other & $\begin{array}{l}\text { Top of lower header (window ledge too narrow) } \\
\text { between } 21 \mathrm{~W} \& 22 \mathrm{~W}\end{array}$ \\
\hline VT515 & $21 \mathrm{~W}-22 \mathrm{~W}$ & $0.10 *$ & 0.20 & Wall & Top of plate between $21 \mathrm{~W} \& 22 \mathrm{~W}$ \\
\hline VT516 & $30 \mathrm{E}$ & $0.17 *$ & 0.34 & Truss & Top of truss about 12 way to the peak from Col. $30 \mathrm{E}$ \\
\hline VT517 & $29-30 \mathrm{E}$ & $0.15 *$ & 0.30 & Bridging & Bridging between Col. 29E \& 30E \\
\hline VT518 & $24-25 W$ & $0.28 *$ & 0.56 & Wall & Top of block wall between Col. $24 \mathrm{~W} \& 25 \mathrm{~W}$ \\
\hline VT520 & $31 \mathrm{~W}$ & $0.17 *$ & 0.34 & Truss & Top of truss near Col. $31 \mathrm{~W}$ \\
\hline VT521 & $30-31 W$ & $0.16 *$ & 0.32 & Bridging & Bridging between Col. 30W \& $31 \mathrm{~W}$ \\
\hline VT522 & $29-30 \mathrm{~W}$ & $0.22 *$ & 0.44 & Bridging & $\begin{array}{l}\text { Bridging about } 12 \text { way to peak from wall between } \\
\text { Col. } 29 \mathrm{~W} \& 30 \mathrm{~W}\end{array}$ \\
\hline VT523 & $27-28 W$ & $0.10 *$ & 0.20 & Bridging & $\begin{array}{l}\text { Bridging about } 2 \backslash 3 \text { way to peak from wall between } \\
\text { Col. } 27 \mathrm{~W} \& 28 \mathrm{~W}\end{array}$ \\
\hline VT524 & $26-27 \mathrm{~W}$ & $0.16 *$ & 0.32 & Bridging & $\begin{array}{l}\text { Bridging about } 34 \text { way to peak from wall between } \\
\text { Col. } 26 W \& 27 W\end{array}$ \\
\hline VT525 & $26-27 E$ & $0.18 *$ & 0.36 & Bridging & $\begin{array}{l}\text { Bridging about } 12 \text { way to peak from wall between } \\
\text { Col. } 26 \mathrm{E} \& 27 \mathrm{E}\end{array}$ \\
\hline
\end{tabular}


Table 4. (continued)

\begin{tabular}{|c|c|c|c|c|c|}
\hline $\begin{array}{l}\text { Sample } \\
\text { ID } a\end{array}$ & $\begin{array}{c}\text { Bay/room/ } \\
\text { structure/ } \\
\text { area }^{b}\end{array}$ & $\begin{array}{c}\mathrm{Be}^{c} \\
(\mu \mathrm{g} / \mathrm{g}) \\
(\mu \mathrm{g})^{*}\end{array}$ & $\begin{array}{c}\text { Be/area } \\
\left(\mu \mathrm{g} / \mathrm{ft}^{2}\right)^{d} \\
\end{array}$ & $\begin{array}{l}\text { Location } \\
\text { in bay }\end{array}$ & Location notese \\
\hline VT526 & $39 \mathrm{~W}$ & $0.22 *$ & 0.44 & Truss & $\begin{array}{l}\text { Top of truss about } 2 \mathrm{ft} \text { away from peak at Col. } 39 \mathrm{~W} \\
\text { after additional cleaning }\end{array}$ \\
\hline VT527 & $22-23 E$ & $0.15 *$ & 0.30 & Bridging & $\begin{array}{l}\text { Bridging, about } 233 \text { way to peak from wall between } \\
\text { Col. } 22 \mathrm{E} \& 23 \mathrm{E}\end{array}$ \\
\hline VT528 & $22 \mathrm{E}$ & $0.13 *$ & 0.26 & Truss & Top of truss about 12 way to peak from Col. $22 \mathrm{E}$ \\
\hline VT529 & $22-23 E$ & $0.10 *$ & 0.20 & Stringer & $\begin{array}{l}\text { Stringer about } 112 \text { way to peak from wall between } \\
\text { Col. } 22 \mathrm{E} \text { \& } 23 \mathrm{E}\end{array}$ \\
\hline VT530 & 22-23E & $0.15 *$ & 0.30 & Bridging & $\begin{array}{l}\text { Bridging about } 14 \text { way to peak from wall between } \\
\text { Col. } 22 \mathrm{E} \& 23 \mathrm{E}\end{array}$ \\
\hline VT531 & $23 \mathrm{E}$ & $0.30 *$ & 0.60 & Truss & Top of truss about $2 \backslash 3$ way to peak from Col. $23 \mathrm{E}$ \\
\hline VT532 & $30 \mathrm{~W}$ & $0.10 *$ & 0.20 & Truss & Top of truss about 12 way to peak from Col. $30 \mathrm{~W}$ \\
\hline VT533 & $29 \mathrm{~W}$ & $0.11 *$ & 0.22 & Truss & Top of truss about $3 \mathrm{~K}$ way to peak from Col. $29 \mathrm{~W}$ \\
\hline VT534 & $29 \mathrm{E}$ & $0.16 *$ & 0.32 & Truss & Top of truss about 12 way to peak from Col. $29 \mathrm{E}$ \\
\hline VT535 & $28-29 E$ & $0.23 *$ & 0.46 & Stringer & $\begin{array}{l}\text { Stringer (upper surface) about } 5 \mathrm{ft} \text { down from peak } \\
\text { between Col. 28E \& 29E peak between Col. 28E \& } \\
29 \mathrm{E}\end{array}$ \\
\hline VT536 & $28-29 E$ & $0.18 *$ & 0.36 & Bridging & $\begin{array}{l}\text { Bridging about } 2 \backslash 3 \text { way to peak from wall between } \\
\text { Col. } 28 \mathrm{E} \& 29 \mathrm{E}\end{array}$ \\
\hline VT537 & $28-29 W$ & $0.17 *$ & 0.34 & Bridging & $\begin{array}{l}\text { Bridging about } 12 \text { way to peak from wall between } \\
\text { Col. } 28 \mathrm{~W} \& 29 \mathrm{~W}\end{array}$ \\
\hline VT538 & $28 \mathrm{~W}$ & $0.29 *$ & 0.58 & Truss & $\begin{array}{l}\text { Top of truss about } 12 \text { way to peak from Col. } 28 \mathrm{~W} \& \\
\text { about } 2 \mathrm{ft} \text { below old VM190 }\end{array}$ \\
\hline VT539 & 27E & $0.10 *$ & 0.20 & Truss & $\begin{array}{l}\text { Top of truss (after extensive recleaning) about } 12 \text { way } \\
\text { to peak from Col. } 27 \mathrm{E}\end{array}$ \\
\hline VT540 & $27 \mathrm{~W}$ & 0.50 * & 1.0 & Truss & $\begin{array}{l}\text { Top of lower horizontal truss brace about } 233 \text { way to } \\
\text { peak from Col. } 27 \mathrm{~W}\end{array}$ \\
\hline VT541 & $26 \mathrm{~W}$ & $0.49 *$ & 0.98 & Truss & $\begin{array}{l}\text { Top of lower horizontal truss member about } 12 \text { way } \\
\text { to peak from Col. } 26 \mathrm{~W}\end{array}$ \\
\hline VT543 & $25-26 \mathrm{E}$ & $0.32 *$ & 0.64 & Bridging & $\begin{array}{l}\text { Bridging about } 12 \text { way to peak from wall between } \\
\text { Col. } 25 \mathrm{E} \& 26 \mathrm{E} \text {. }\end{array}$ \\
\hline VT544 & $25 \mathrm{E}$ & $0.21 *$ & 0.42 & Truss & Top of truss about $2 \backslash 3$ way to peak from Col. $25 \mathrm{E}$ \\
\hline VT545 & $24-25 E$ & $0.35 *$ & 0.70 & Bridging & $\begin{array}{l}\text { Bridging about } 2 \backslash 3 \text { way to peak from wall between } \\
\text { Col. } 24 \mathrm{E} \& 25 \mathrm{E}\end{array}$ \\
\hline VT546 & $25 \mathrm{~W}$ & $0.29 *$ & 0.58 & Truss & Top of truss about $5 \mathrm{ft}$ from peak on Col. line $25 \mathrm{~W}$ \\
\hline VT547 & $24-25 W$ & $0.44 *$ & 0.88 & Bridging & $\begin{array}{l}\text { Bridging about } 2 \mathrm{ft} \text { from peak between Col. } 24 \mathrm{~W} \& \\
25 \mathrm{~W}\end{array}$ \\
\hline
\end{tabular}


Table 4. (continued)

\begin{tabular}{|c|c|c|c|c|c|}
\hline $\begin{array}{l}\text { Sample } \\
\text { ID } a\end{array}$ & $\begin{array}{c}\text { Bay/room/ } \\
\text { structure/ } \\
\text { area }^{b}\end{array}$ & $\begin{array}{c}\mathrm{Be}^{c} \\
(\mu \mathrm{g} / \mathrm{g}) \\
(\mu \mathrm{g})^{*}\end{array}$ & $\begin{array}{c}\text { Be/area } \\
\left(\mu \mathrm{g} / \mathrm{ft}^{2}\right)^{d}\end{array}$ & $\begin{array}{l}\text { Location } \\
\text { in bay }\end{array}$ & Location notese \\
\hline VT548 & $24 \mathrm{E}$ & $0.46 *$ & 0.92 & Wall & $\begin{array}{l}\text { Top of plate above truss about } 4 \mathrm{ft} \text { from metal shop } \\
\text { roof peak on Col. line } 24 \mathrm{E}\end{array}$ \\
\hline VT549 & $24 \mathrm{~W}$ & $0.63 *$ & 1.3 & Truss & $\begin{array}{l}\text { Top of truss at roof line step-down between metal } \\
\text { shop \& carpenter end }\end{array}$ \\
\hline VT551 & 23-24E & $0.87 *$ & 1.7 & Bridging & $\begin{array}{l}\text { Bridging about } 112 \text { way to peak between Col. } 23 \mathrm{E} \& \\
24 \mathrm{E}\end{array}$ \\
\hline VT552 & $23 \mathrm{~W}$ & $0.21 *$ & 0.42 & Truss & $\begin{array}{l}\text { Top of truss about } 12 \text { way to peak from Col. } 23 \mathrm{~W} \\
\text { (about } 2 \mathrm{ft} \text { below old VM395) }\end{array}$ \\
\hline VT553 & $22-23 W$ & $0.14 *$ & 0.28 & Bridging & $\begin{array}{l}\text { Bridging about } 112 \text { way to peak between Col. } 22 \mathrm{~W} \& \\
23 \mathrm{~W}\end{array}$ \\
\hline VT554 & $22 \mathrm{~W}$ & $0.11 *$ & 0.22 & Truss & Top of truss about $2 \backslash 3$ way to peak from Col. $22 \mathrm{~W}$ \\
\hline VT555 & $21-22 W$ & $0.15 *$ & 0.30 & Bridging & $\begin{array}{l}\text { Bridging about } 12 \text { way to peak between Col. } 21 \mathrm{~W} \& \\
22 \mathrm{~W} \text { (metal-shop side of wall) }\end{array}$ \\
\hline VT556 & $31 \mathrm{~W}$ & $0.23 *$ & 0.46 & Floor & $\begin{array}{l}\text { Crane rail track on floor near Col. } 31 \mathrm{~W} \text {. Further } \\
\text { cleaning after old VM } 443\end{array}$ \\
\hline VT557 & $42 W$ & $0.20 *$ & 0.40 & Floor & $\begin{array}{l}\text { Col. } 42 \mathrm{~W} \text { after add.exten.cleaning Col. } 42 \mathrm{~W} \text { after } \\
\text { add.exten.cleaning }\end{array}$ \\
\hline VT558 & $38 \mathrm{E}$ & $0.15 *$ & 0.30 & Floor & $\begin{array}{l}\text { Crane rail track on floor } 3 \mathrm{ft} \text { directly } \mathrm{W} \text { of Col. } 38 \mathrm{E} \\
\text { after exten.add.cleaning }\end{array}$ \\
\hline VT559 & $38 \mathrm{~W}$ & $0.14 *$ & 0.56 & Floor & $\begin{array}{l}\text { Depression in floor } 20 \mathrm{ft} \& 7 \mathrm{in} . \mathrm{E} \text { and } 5.33 \mathrm{ft} \mathrm{N} \text { of } \\
\text { Col. } 38 \mathrm{~W} \text { post add.clean. }\end{array}$ \\
\hline VT560 & $27 \mathrm{E}$ & $0.34 *$ & 0.68 & Floor & $\begin{array}{l}\text { Crane rail track on floor } 3 \mathrm{ft} \text { directly } \mathrm{W} \text { of Col. } 27 \mathrm{E} \\
\text { post exten.add. cleaning }\end{array}$ \\
\hline VT561 & $22 W$ & $0.17 *$ & 0.68 & Other & $\begin{array}{l}\text { Upper horizontal surface of original electrical fuse box } \\
33 \mathrm{ft} \mathrm{S} \text { of Col. } 22 \mathrm{~W}\end{array}$ \\
\hline VT562 & $20 \mathrm{~W}$ & $0.11 *$ & 0.22 & Floor & $\begin{array}{l}\text { Floor (original concrete) located } 3 \mathrm{ft} \text { directly } S \text { of and } \\
\mathrm{N} \text { of Col. } 17 \mathrm{~W} \text { Col. } 20 \mathrm{~W}\end{array}$ \\
\hline VT563 & $17 \mathrm{~W}$ & $0.14 *$ & 0.28 & Floor & $\begin{array}{l}\text { Floor (original concrete) located directly against and } \mathrm{N} \\
\text { of Col. } 17 \mathrm{~W}\end{array}$ \\
\hline VT564 & $14 \mathrm{~W}$ & $0.12 *$ & 0.12 & Floor & $\begin{array}{l}\text { Floor (original concrete) located directly against and } \mathrm{N} \\
\text { of Col. } 14 \mathrm{~W}\end{array}$ \\
\hline VT565 & $15 \mathrm{~W}$ & $0.12 *$ & 0.48 & Other & $\begin{array}{l}\text { Upper horizontal surface of original (?) electrical fuse } \\
\text { box } 1 \mathrm{ft} \mathrm{N} \text { of Col. } 15 \mathrm{~W}\end{array}$ \\
\hline VT566 & $16 \mathrm{~W}$ & $0.10^{*}$ & 0.10 & Floor & $\begin{array}{l}\text { Floor (original concrete) located directly against and } \mathrm{N} \\
\text { of Col. } 16 \mathrm{~W}\end{array}$ \\
\hline VT567 & $20-21 \mathrm{E}$ & $1.0^{*}$ & 2.0 & Wall & $\begin{array}{l}\text { Top of block wall in middle of the bay. VT801 is } \\
\text { recheck. }\end{array}$ \\
\hline
\end{tabular}


Table 4. (continued)

\begin{tabular}{|c|c|c|c|c|c|}
\hline $\begin{array}{l}\text { Sample } \\
\text { ID }^{a}\end{array}$ & $\begin{array}{c}\text { Bay/room/ } \\
\text { structure/ } \\
\text { area } b\end{array}$ & $\begin{array}{c}\mathrm{Be}^{c} \\
(\mu \mathrm{g} / \mathrm{g}) \\
(\mu \mathrm{g})^{*}\end{array}$ & $\begin{array}{l}\text { Be/area } \\
\left(\mu \mathrm{g} / \mathrm{ft}^{2}\right)^{d}\end{array}$ & $\begin{array}{c}\text { Location } \\
\text { in bay }\end{array}$ & Location notese \\
\hline VT568 & $21 E$ & $0.11 *$ & 0.22 & Truss & $\begin{array}{l}\text { Top of north Truss } 21,113 \text { of the way up towards } \\
\text { peak }\end{array}$ \\
\hline VT569 & $20-21 E$ & $0.07 *$ & 0.14 & Bridging & $\begin{array}{l}\text { Bridging } 23 \text { of the way up towards peak in middle of } \\
\text { bay }\end{array}$ \\
\hline VT570 & $19-20 \mathrm{E}$ & $0.14 *$ & 0.28 & Wall & Top of block wall; middle of bay \\
\hline VT571 & $19-20 \mathrm{E}$ & $0.07 *$ & 0.14 & Bridging & Bridging 13 way up towards peak \\
\hline VT572 & $19-20 \mathrm{E}$ & $0.09 *$ & 0.18 & Truss & Top of Truss $20,2 \backslash 3$ way up towards peak \\
\hline VT573 & $18-19 \mathrm{E}$ & $0.08 *$ & 0.16 & Wall & Top of block wall, middle of bay \\
\hline VT574 & $18-19 \mathrm{E}$ & $0.08 *$ & 0.16 & Bridging & $\begin{array}{l}\text { Bridging } 13 \text { of the way up to peak between Stringers } \\
8 \& 9\end{array}$ \\
\hline VT575 & $18-19 E$ & $0.08 *$ & 0.16 & Truss & $\begin{array}{l}\text { Top of Truss } 19,2 \backslash 3 \text { way up to peak between } \\
\text { Stringers } 14 \& 15\end{array}$ \\
\hline VT576 & $17-18 E$ & $0.08 *$ & 0.16 & Wall & Top of block wall, middle of bay \\
\hline VT577 & $17-18 \mathrm{E}$ & $0.08 *$ & 0.16 & Bridging & $\begin{array}{l}\text { Bridging } 13 \text { way up towards peak betw. Stringers } 8 \& \\
9\end{array}$ \\
\hline VT578 & $18 \mathrm{E}$ & $0.09 *$ & 0.18 & Truss & Top of Truss $18,2 \backslash 3$ way up towards peak \\
\hline VT579 & $16-17 \mathrm{E}$ & $0.07 *$ & 0.14 & Wall & Top of block wall, middle of bay \\
\hline VT580 & $16-17 \mathrm{E}$ & $0.14 *$ & 0.28 & Bridging & Bridging (S) 13 way up towards peak \\
\hline VT581 & $17 \mathrm{E}$ & $0.08 *$ & 0.16 & Truss & Top of Truss 17,213 of the way up towards peak \\
\hline VT582 & $8-9 \mathrm{~W}$ & $0.08 *$ & 0.16 & Wall & $\begin{array}{l}\text { Top of block wall, south wall of bldg. (WS), } 13 \text { of } \\
\text { the way over towards peak }\end{array}$ \\
\hline VT583 & $8-9 \mathrm{~W}$ & $0.08 *$ & 0.08 & Wall & $\begin{array}{l}\text { Top of truss support beam (runs } N \text { to } S \text { ), middle of } \\
\text { bay. }\end{array}$ \\
\hline VT584 & $8-9 \mathrm{~W}$ & $0.08 *$ & 0.16 & Bridging & Bridging $(N), 13$ of the way up towards peak \\
\hline VT585 & $9 \mathrm{~W}$ & $0.09 *$ & 0.18 & Truss & Top of Truss 9,23 way up towards peak \\
\hline VT586 & $8 w$ & $0.15 *$ & 0.30 & Truss & Top of Truss 8,13 way of the way up towards peak \\
\hline VT587 & $9-10 \mathrm{~W}$ & $0.12 *$ & 0.12 & Truss & $\begin{array}{l}\text { Top of the truss support beam ( } N \text { to } S \text { ), mid. of the } \\
\text { bay }\end{array}$ \\
\hline VT588 & $9-10 \mathrm{~W}$ & $0.09 *$ & 0.18 & Bridging & Bridging (N) about $3 \mathrm{ft}$ away from the roof peak \\
\hline VT589 & $10 \mathrm{~W}$ & $0.09 *$ & 0.18 & Truss & Top of Truss 10,12 of the way up towards peak \\
\hline VT590 & $10-11 \mathrm{~W}$ & $0.08 *$ & 0.08 & Truss & Top of truss support beam ( $\mathrm{N}$ to $\mathrm{S}$ ), middle of bay \\
\hline VT591 & $10-11 \mathrm{~W}$ & $0.09 *$ & 0.18 & Bridging & Bridging $(S), 12$ of the way up towards peak \\
\hline VT592 & $11 \mathrm{~W}$ & $0.09 *$ & 0.18 & Truss & Top of Truss 11,23 of the way up towards peak \\
\hline VT593 & $12-13 W$ & $0.08 *$ & 0.16 & Bridging & Bridging $(S), 12$ way up towards peak \\
\hline
\end{tabular}


Table 4. (continued)

\begin{tabular}{|c|c|c|c|c|c|}
\hline $\begin{array}{l}\text { Sample } \\
\text { IDa }\end{array}$ & $\begin{array}{c}\text { Bay/room/ } \\
\text { structure/ } \\
\text { area }^{b}\end{array}$ & $\begin{array}{c}\mathrm{Bec} \\
(\mu \mathrm{g} / \mathrm{g}) \\
(\mu \mathrm{g})^{*}\end{array}$ & $\begin{array}{c}\text { Be/area } \\
\left(\mu \mathrm{g} / \mathrm{ft}^{2}\right)^{d}\end{array}$ & $\begin{array}{l}\text { Location } \\
\text { in bay }\end{array}$ & Location notese \\
\hline VT594 & $12 \mathrm{~W}$ & $0.09 *$ & 0.18 & Truss & Top of Truss 12,12 the way up towards the peak \\
\hline VT595 & $12-13 W$ & $0.08 *$ & 0.16 & Wall & Top of block wall, middle of bay \\
\hline VT596 & $11-12 W$ & $0.08 *$ & 0.16 & Truss & $\begin{array}{l}\text { Top of the truss support beam, middle of bay. This is } \\
\text { same as top of block wall. }\end{array}$ \\
\hline VT597 & $8 \mathrm{E}$ & $0.08 *$ & 0.16 & Wall & $\begin{array}{l}\text { Top of block wall (wood plate), W of Col. } 8 \mathrm{E} \text { (SE } \\
\text { corner) }\end{array}$ \\
\hline VT598 & $8 \mathrm{E}$ & $0.09 *$ & 0.18 & Truss & Top of truss, about $3 \mathrm{~K}$ way to the peak from Col. $8 \mathrm{E}$ \\
\hline VT599 & $8 \mathrm{E}-9 \mathrm{E}$ & $0.09 *$ & 0.18 & Bridging & Bridging, 3\ way to the peak between Col.8E \& 9E \\
\hline VT600 & $8 \mathrm{E}-9 \mathrm{E}$ & $0.11 *$ & 0.22 & Wall & Top of block wall (wood plate), between $8 \mathrm{E} \& 9 \mathrm{E}$ \\
\hline VT601 & $9 \mathrm{E}-10 \mathrm{E}$ & $0.09 *$ & 0.18 & Wall & Top of block wall (wood plate), between $9 \mathrm{E} \& 10 \mathrm{E}$ \\
\hline VT602 & $9 E-10 E$ & $0.10 *$ & 0.20 & Bridging & $\begin{array}{l}\text { Bridging, about } 13 \text { way to the peak between } 9 \mathrm{E} \\
\& 10 \mathrm{E}\end{array}$ \\
\hline VT603 & $9 E$ & $0.08 *$ & 0.16 & Truss & Top of truss, about $3 \mathrm{~W}$ way to peak from Col. $9 \mathrm{E}$ \\
\hline VT604 & $10 \mathrm{E}$ & $0.08 *$ & 0.16 & Truss & Top of truss, about $3 \mathrm{M}$ way to peak from Col. $10 \mathrm{E}$ \\
\hline VT605 & $10 \mathrm{E}-11 \mathrm{E}$ & $0.08 *$ & 0.16 & Bridging & Bridging, about $2 \backslash 3$ way to peak between $10 E$ \& $11 E$ \\
\hline VT606 & $10 \mathrm{E}-11 \mathrm{E}$ & $0.08 *$ & 0.16 & Wall & Top of block wall (wood plate), between 10E \& $11 \mathrm{E}$ \\
\hline VT607 & $11 E-12 E$ & $0.08 *$ & 0.16 & Bridging & Bridging, about 13 way to peak between $11 \mathrm{E} \& 12 \mathrm{E}$ \\
\hline VT608 & $11 \mathrm{E}$ & $0.08 *$ & 0.16 & Truss & Top of truss, about 13 way to peak from Col. $11 \mathrm{E}$ \\
\hline VT609 & $11 E-12 E$ & $0.07 *$ & 0.14 & Wall & Top of block wall between $11 \mathrm{E} \& 12 \mathrm{E}$ \\
\hline VT610 & $12 \mathrm{E}$ & $0.09 *$ & 0.18 & Truss & Top of truss, about 12 way to peak from Col.12E \\
\hline VT611 & $12 E-13 E$ & $0.08 *$ & 0.16 & Wall & Top of block wall (wood plate), between 12E \& 13E \\
\hline VT612 & $12 E-13 E$ & $0.08 *$ & 0.16 & Bridging & Bridging, about 12 way to peak between $12 \mathrm{E} \& 13 \mathrm{E}$ \\
\hline VT613 & $13 \mathrm{E}$ & $0.08 *$ & 0.16 & Truss & Top of truss, about $3 \mathrm{~K}$ way to peak from Col. 13E \\
\hline VT614 & $13 E-14 E$ & $0.07 *$ & 0.14 & Wall & Top of block wall (wood plate), between 13E \& 14E \\
\hline VT615 & $13 E-14 E$ & $0.08 *$ & 0.16 & Bridging & Bridging, about 12 way to peak between $13 E$ \& 14E \\
\hline VT616 & $14 \mathrm{E}$ & $0.08 *$ & 0.16 & Truss & Top of truss, about 23 way to peak from $14 \mathrm{E}$ \\
\hline VT617 & $13 E-14 E$ & $0.08 *$ & 0.08 & Stringer & $\begin{array}{l}\text { Stringer (upper side), about } 23 \text { way to peak between } \\
13 E \& 14 E\end{array}$ \\
\hline VT618 & $15 \mathrm{E}$ & $0.08 *$ & 0.16 & Truss & Top of truss, about 34 way to peak from $15 E$ \\
\hline VT619 & $14 \mathrm{E}-15 \mathrm{E}$ & $0.14 *$ & 0.28 & Bridging & Bridging, about $3 \mathrm{M}$ way to peak between $14 \mathrm{E} \& 15 \mathrm{E}$ \\
\hline VT620 & $14 E-15 E$ & $0.14 *$ & 0.28 & Wall & Top of block wall (block) between 14E \& 15E \\
\hline VT621 & $15 \mathrm{E}-16 \mathrm{E}$ & $0.07 *$ & 0.14 & Wall & Top of block wall (wood plate), between $15 \mathrm{E} \& 16 \mathrm{E}$ \\
\hline VT622 & $15 \mathrm{E}-16 \mathrm{E}$ & $0.08 *$ & 0.16 & Bridging & Bridging, about 13 way to peak between $15 \mathrm{E} \& 16 \mathrm{E}$ \\
\hline
\end{tabular}


Table 4. (continued)

\begin{tabular}{|c|c|c|c|c|c|}
\hline $\begin{array}{l}\text { Sample } \\
\text { ID } a\end{array}$ & $\begin{array}{c}\text { Bay/room/ } \\
\text { structure/ } \\
\text { area } b\end{array}$ & $\begin{array}{c}\mathrm{Be}^{c} \\
(\mu \mathrm{g} / \mathrm{g}) \\
(\mu \mathrm{g})^{*}\end{array}$ & $\begin{array}{c}\text { Be/area } \\
\left(\mu \mathrm{g} / \mathrm{ft}^{2}\right)^{d} \\
\end{array}$ & $\begin{array}{l}\text { Location } \\
\text { in bay }\end{array}$ & Location notese \\
\hline VT623 & $16 \mathrm{E}$ & $0.07 *$ & 0.14 & Truss & Top of truss, about 12 way to peak from $16 \mathrm{E}$ \\
\hline VT624 & $17 \mathrm{~W}-18 \mathrm{~W}$ & $0.08 *$ & 0.16 & Bridging & Bridging, about $3 \mathrm{M}$ way to peak between $17 \mathrm{~W} \& 18 \mathrm{~W}$ \\
\hline VT625 & $18 \mathrm{~W}$ & $0.17 *$ & 0.34 & Truss & Top of truss, about 12 way to peak from $18 \mathrm{~W}$ \\
\hline VT626 & $17 \mathrm{~W}$ & $0.08 *$ & 0.16 & Truss & Top of truss, about 314 way to peak from $17 \mathrm{~W}$ \\
\hline VT627 & $20 \mathrm{~W}-21 \mathrm{~W}$ & $0.09 *$ & 0.18 & Wall & Top of block wall (wood plate), between $20 \mathrm{~W} \& 21 \mathrm{~W}$ \\
\hline VT628 & $19 W-20 W$ & $0.07 *$ & 0.14 & Wall & Top of block wall (wood plate), between $19 \mathrm{~W} \& 20 \mathrm{~W}$ \\
\hline VT629 & $18 W-19 W$ & $0.08 *$ & 0.16 & Wall & Top of block wall (wood plate), between $18 \mathrm{~W} \& 19 \mathrm{~W}$ \\
\hline VT630 & $17 W-18 W$ & $0.08 *$ & 0.16 & Wall & Top of block wall (block), between $17 \mathrm{~W} \& 18 \mathrm{~W}$ \\
\hline VT631 & $21 \mathrm{~W}$ & $0.08 *$ & 0.16 & Truss & Top of truss, about 13 way to peak Col. $21 \mathrm{~W}$ \\
\hline VT632 & $20 W-21 W$ & $0.07 *$ & 0.14 & Bridging & Bridging, about 113 way to peak between $20 \mathrm{~W} \& 21 \mathrm{~W}$ \\
\hline VT633 & $20 \mathrm{~W}$ & $0.13 *$ & 0.26 & Truss & Top of truss, about $3 \mathrm{~K}$ way to peak from Col.20W \\
\hline VT634 & $19 \mathrm{~W}-20 \mathrm{~W}$ & $0.08 *$ & 0.16 & Bridging & Bridging, about 13 way to peak between $19 \mathrm{~W} \& 20 \mathrm{~W}$ \\
\hline VT635 & $19 \mathrm{~W}$ & $0.13 *$ & 0.26 & Truss & Top of truss, about 113 way to peak from Col. $19 \mathrm{~W}$ \\
\hline VT636 & $18 W-19 W$ & $0.07 *$ & 0.14 & Bridging & Bridging, about 13 way to peak between $18 \mathrm{~W} \& 19 \mathrm{~W}$ \\
\hline VT637 & $16 \mathrm{~W}-17 \mathrm{~W}$ & $0.08 *$ & 0.16 & Wall & Top of block wall (block), between 16W \& $17 \mathrm{~W}$ \\
\hline VT638 & $16 \mathrm{~W}-17 \mathrm{~W}$ & $0.16 *$ & 0.32 & Bridging & Bridging, about 23 way to peak between $16 \mathrm{~W} \& 17 \mathrm{~W}$ \\
\hline VT639 & $16 \mathrm{~W}-17 \mathrm{~W}$ & $0.08 *$ & 0.08 & Stringer & $\begin{array}{l}\text { Stringer (upper vertical surface), about } 2 \backslash 3 \text { way to } \\
\text { peak between } 16 \mathrm{~W} \& 17 \mathrm{~W}\end{array}$ \\
\hline VT640 & $16 \mathrm{~W}$ & $0.07 *$ & 0.14 & Truss & Top of truss, about $2 \backslash 3$ way to peak from $16 \mathrm{~W}$ \\
\hline VT641 & $15 W-16 W$ & $0.08 *$ & 0.16 & Bridging & Bridging, about 314 way to peak between $15 \mathrm{~W} \& 16 \mathrm{~W}$ \\
\hline VT642 & $15 \mathrm{~W}$ & $0.07 *$ & 0.14 & Truss & Top of truss, about $3 \mathrm{~V}$ way to peak from $15 \mathrm{~W}$ \\
\hline VT643 & $15 \mathrm{~W}-16 \mathrm{~W}$ & $<0.05 *$ & $<0.10$ & Wall & Top of block wall (wood plate), between $15 \mathrm{~W} \& 16 \mathrm{~W}$ \\
\hline VT644 & $14 W-15 W$ & $<0.05 *$ & $<0.10$ & Wall & $\begin{array}{l}\text { Top of block wall (block \& wet from localized roof } \\
\text { leak) between } 14 \mathrm{~W} \& 15 \mathrm{~W}\end{array}$ \\
\hline VT645 & $14 W-15 W$ & $<0.05 *$ & $<0.10$ & Bridging & Bridging, about 12 way to peak between $14 \mathrm{~W} \& 15 \mathrm{~W}$ \\
\hline VT646 & $14 \mathrm{~W}$ & $<0.05 *$ & $<0.10$ & Truss & Top of truss, about 12 way to peak from $14 \mathrm{~W}$ \\
\hline VT647 & $13 W-14 W$ & $<0.05 *$ & $<0.10$ & Bridging & Bridging, about $3 \mathrm{~K}$ way to peak between $13 \mathrm{~W} \& 14 \mathrm{~W}$ \\
\hline VT648 & $13 \mathrm{~W}$ & $<0.05 *$ & $<0.10$ & Truss & $\begin{array}{l}\text { Top of truss, about } \lambda 8 \text { ( } 6 \mathrm{ft} \text { down from peak) way to } \\
\text { peak from Col. } 13 \mathrm{~W}\end{array}$ \\
\hline VT649 & $13 W-14 W$ & $<0.05 *$ & $<0.10$ & Wall & Top of block wall (block), between 13W \& 14W \\
\hline VT650 & $6 S-7 S$ & $<0.05 *$ & $<0.10$ & Wall & Top of block wall (block), between $6 S \& 7 S$ \\
\hline VT651 & $6 S-7 S$ & $<0.05 *$ & $<0.10$ & Bridging & Bridging, about 12 way to peak between $6 S \& 7 S$ \\
\hline
\end{tabular}


Table 4. (continued)

\begin{tabular}{|c|c|c|c|c|c|}
\hline $\begin{array}{l}\text { Sample } \\
\text { ID } a\end{array}$ & $\begin{array}{c}\text { Bay/room/ } \\
\text { structure/ } \\
\text { area }^{b}\end{array}$ & $\begin{array}{c}\mathrm{Be}^{c} \\
(\mu \mathrm{g} / \mathrm{g}) \\
(\mu \mathrm{g})^{*}\end{array}$ & $\begin{array}{l}\text { Be/area } \\
\left(\mu \mathrm{g} / \mathrm{ft}^{2}\right)^{d}\end{array}$ & $\begin{array}{l}\text { Location } \\
\text { in bay }\end{array}$ & Location notese \\
\hline VT652 & $6 S$ & $<0.05^{*}$ & $<0.10$ & Truss & $\begin{array}{l}\text { Top of truss, about } 78 \text { way to peak ( } 10 \mathrm{ft} \text { down) from } \\
\text { Col. } 6 \mathrm{~S}\end{array}$ \\
\hline VT653 & $7 S$ & $<0.05 *$ & $<0.10$ & Truss & $\begin{array}{l}\text { Top of truss, about } 3 / 4 \text { way to peak from Col. } 7 \mathrm{~S} \text { (to } \\
\text { Col. } \mathrm{TN} \text { ) in triangle }\end{array}$ \\
\hline VT654 & $7 \mathrm{~S}$ & $<0.05 *$ & $<0.10$ & Truss & $\begin{array}{l}\text { Top of truss, about } 1 / 2 \text { way to peak from Col. } 7 S \text { (to } \\
\text { Col. } 12 W \text { ) in triangle }\end{array}$ \\
\hline VT655 & $7 S$ & $<0.05^{*}$ & $<0.05$ & Stringer & $\begin{array}{l}\text { Stringer (upper vertical surface), about } 12 \text { way to } \\
\text { peak (in triangle) from Col. } 7 \mathrm{~S}\end{array}$ \\
\hline VT656 & $6 N-7 N$ & $<0.05 *$ & $<0.10$ & Bridging & $\begin{array}{l}\text { Bridging, about } 3 \backslash 4 \text { way to peak between Col. } 6 \mathrm{~N} \& \\
7 \mathrm{~N}\end{array}$ \\
\hline VT657 & $6 \mathrm{~N}$ & $<0.05 *$ & $<0.10$ & Truss & Top of truss, about 344 way to peak from Col. $6 \mathrm{~N}$ \\
\hline VT658 & $6 \mathrm{~N}-7 \mathrm{~N}$ & $<0.05 *$ & $<0.10$ & Wall & Top of block wall (wood plate), between $6 \mathrm{~N} \& 7 \mathrm{~N}$ \\
\hline VT659 & $6 \mathrm{~N}-7 \mathrm{~N}$ & $<0.05 *$ & $<0.10$ & Window & Window ledge (wood), between $6 \mathrm{~N} \& 7 \mathrm{~N}$ \\
\hline VT660 & $7 \mathrm{~N}-12 \mathrm{~W}$ & $<0.05 *$ & $<0.10$ & Window & $\begin{array}{l}\text { Window ledge (wood), between } 7 \mathrm{~N} \& 12 \mathrm{~W} \text { (in } \\
\text { triangle) }\end{array}$ \\
\hline VT661 & $7 \mathrm{~N}-12 \mathrm{~W}$ & $0.07 *$ & 0.14 & Wall & $\begin{array}{l}\text { Top of block wall (wood plate), between } 7 \mathrm{~N} \& 12 \mathrm{~W} \\
\text { (in triangle) }\end{array}$ \\
\hline VT662 & $7 N-12 W$ & $<0.05 *$ & $<0.10$ & Bridging & $\begin{array}{l}\text { Bridging, about } 13 \text { way to peak between } 7 \mathrm{~N} \& 12 \mathrm{~W} \\
\text { (in triangle) }\end{array}$ \\
\hline VT663 & $7 \mathrm{~N}$ & $<0.05^{*}$ & $<0.10$ & Truss & Top of truss, about 13 way to peak from Col. $7 \mathrm{~N}$ \\
\hline VT664 & $12 W$ & $<0.05 *$ & $<0.10$ & Truss & $\begin{array}{l}\text { Top of truss, about } 3 / 4 \text { way to peak going toward } 7 S \\
\text { from Col. } 12 W \text { (in triangle) }\end{array}$ \\
\hline VT665 & $12 W-13 W$ & $<0.05 *$ & $<0.10$ & Window & Window ledge (wood), between $12 W \& 13 W$ \\
\hline VT666 & $13 W-14 W$ & $<0.05 *$ & $<0.10$ & Window & Window ledge (wood), between $13 W$ \& $14 W$ \\
\hline VT667 & $14 \mathrm{~W}-15 \mathrm{~W}$ & $<0.05 *$ & $<0.10$ & Window & Window ledge (wood) between $14 \& 15 \mathrm{~W}$ \\
\hline VT668 & $15 \mathrm{~W}-16 \mathrm{~W}$ & $<0.05 *$ & $<0.05$ & Window & Window ledge (wood) between $15 \mathrm{~W} \& 16 \mathrm{~W}$ \\
\hline VT669 & $16 \mathrm{~W}-17 \mathrm{~W}$ & $<0.05^{*}$ & $<0.10$ & Window & Window ledge (wood) between $16 \mathrm{~W} \& 17 \mathrm{~W}$ \\
\hline VT670 & $17 W-18 W$ & $<0.05 *$ & $<0.10$ & Window & Window ledge (wood) between $17 \mathrm{~W} \& 18 \mathrm{~W}$ \\
\hline VT671 & $11-12 W$ & $0.08 *$ & 0.16 & Bridging & Bridging, 12 of the way up towards the peak \\
\hline VT672 & $20-21 \mathrm{E}$ & $0.07 *$ & 0.07 & Floor & Floor, $9.5 \mathrm{ft} \mathrm{S} \mathrm{\&} 10 \mathrm{ft} \mathrm{W}$ of column Beam 21 \\
\hline VT673 & 19-20E & $0.07 *$ & 0.07 & Floor & Floor, $9.5 \mathrm{ft} \mathrm{S} \mathrm{\&} 10 \mathrm{ft} \mathrm{W}$ of column Beam 20 \\
\hline VT674 & $18-19 E$ & $0.17 *$ & 0.17 & Floor & Floor, $9.5 \mathrm{ft} \mathrm{S} \& 10 \mathrm{ft}$ W of column Beam 19 \\
\hline VT675 & $17-18 \mathrm{E}$ & $0.15 *$ & 0.15 & Floor & Floor, $9.5 \mathrm{ft} \mathrm{S} \mathrm{\&} 10 \mathrm{ft} \mathrm{W}$ of column Beam 18 \\
\hline VT676 & $16-17 \mathrm{E}$ & $0.14 *$ & 0.14 & Floor & Floor, $9.5 \mathrm{ft} \mathrm{S} \mathrm{\&} 10 \mathrm{ft} \mathrm{W}$ of column Beam 17 \\
\hline
\end{tabular}


Table 4. (continued)

\begin{tabular}{|c|c|c|c|c|c|}
\hline $\begin{array}{c}\text { Sample } \\
\text { ID } a\end{array}$ & $\begin{array}{c}\text { Bay/room/ } \\
\text { structure/ } \\
\text { areab }\end{array}$ & $\begin{array}{c}\mathrm{Be}^{c} \\
(\mu \mathrm{g} / \mathrm{g}) \\
(\mu \mathrm{g})^{*}\end{array}$ & $\begin{array}{r}\text { Be/area } \\
\left(\mu \mathrm{g} / \mathrm{ft}^{2}\right)^{d} \\
\end{array}$ & $\begin{array}{l}\text { Location } \\
\text { in bay }\end{array}$ & Location notese \\
\hline VT677 & $15-16 \mathrm{E}$ & $0.16 *$ & 0.16 & Floor & Floor, $9.5 \mathrm{ft} \mathrm{S} \& 10 \mathrm{ft} \mathrm{W}$ of column Beam 16 \\
\hline VT678 & $14-15 E$ & $0.16 *$ & 0.16 & Floor & Floor, $9.5 \mathrm{ft} \mathrm{S} \& 10 \mathrm{ft} W$ of column Beam 15 \\
\hline VT679 & $13-14 E$ & $0.07 *$ & 0.07 & Floor & Floor, $9.5 \mathrm{ft} \mathrm{S} \mathrm{\&} 10 \mathrm{ft} \mathrm{W}$ of Col. 14 \\
\hline VT680 & 12-13E & $0.08 *$ & 0.08 & Floor & Floor, $9.5 \mathrm{ft} \mathrm{S} \mathrm{\&} 10 \mathrm{ft} \mathrm{W}$ of Col. 13 \\
\hline VT681 & $11-12 E$ & $0.07 *$ & 0.07 & Floor & Floor, $5.5 \mathrm{ft} \mathrm{S} \mathrm{\&} 10 \mathrm{ft} \mathrm{W}$ of Col. 12 \\
\hline VT682 & $10-11 E$ & $0.07 *$ & 0.07 & Floor & Floor, $9.5 \mathrm{ft} \mathrm{S} \mathrm{\&} 10 \mathrm{ft} \mathrm{W}$ of Col. 11 \\
\hline VT683 & $9-10 \mathrm{E}$ & $0.06 *$ & 0.06 & Floor & Floor, $9.5 \mathrm{ft} \mathrm{S} \mathrm{\&} 10 \mathrm{ft} W$ of Col. 10 \\
\hline VT684 & $8-9 \mathrm{~W}$ & $0.15 *$ & 0.15 & Floor & Floor, $9.5 \mathrm{ft} \mathrm{S} \& 10 \mathrm{ft} E$ of Col. 9 \\
\hline VT685 & $9-10 \mathrm{~W}$ & $0.08 *$ & 0.08 & Floor & Floor, $9.5 \mathrm{ft} \mathrm{S} \mathrm{\&} 10 \mathrm{ft} E$ of Col. 10 \\
\hline VT686 & $10-11 \mathrm{~W}$ & $0.15 *$ & 0.15 & Floor & Floor, $9.5 \mathrm{ft} \mathrm{S} \mathrm{\&} 10 \mathrm{ft} E$ of Col. 11 \\
\hline VT687 & $11-12 \mathrm{~W}$ & $0.07 *$ & 0.07 & Floor & Floor, $9.5 \mathrm{ft} \mathrm{S} \& 10 \mathrm{ft} \mathrm{E}$ of Col. 12 \\
\hline VT688 & $12-13 W$ & $0.16^{*}$ & 0.16 & Floor & Floor, $9.5 \mathrm{~S} \& 26 \mathrm{ft} \mathrm{E}$ of Col. 13 \\
\hline VT689 & $13-14 \mathrm{~W}$ & $<0.05 *$ & $<0.05$ & Floor & Floor, $9.5 \mathrm{ft} \mathrm{S} \mathrm{\&} 26 \mathrm{ft} \mathrm{E}$ of Col. 14 \\
\hline VT690 & $14-15 W$ & $<0.05 *$ & $<0.05$ & Floor & Floor, $9.5 \mathrm{ft} \mathrm{S} \mathrm{\&} 26 \mathrm{ft}$ E of Col. 15 \\
\hline VT691 & $15-16 \mathrm{~W}$ & $<0.05 *$ & $<0.05$ & Floor & Floor, $9.5 \mathrm{ft} \mathrm{S} \mathrm{\&} 26 \mathrm{ft}$ E of Col. 16 \\
\hline VT692 & $16-17 \mathrm{~W}$ & $<0.05 *$ & $<0.05$ & Floor & Floor, $9.5 \mathrm{ft} \mathrm{S} \mathrm{\&} 32 \mathrm{ft} \mathrm{E}$ of Col. 17 \\
\hline VT693 & $17-18 \mathrm{~W}$ & $<0.05 *$ & $<0.05$ & Floor & Floor, $9.5 \mathrm{ft} \mathrm{S} \& 32 \mathrm{ft}$ E of Col. 18 \\
\hline VT694 & $18-19 W$ & $0.05 *$ & 0.05 & Floor & Floor, $9.5 \mathrm{ft} \mathrm{S} \mathrm{\&} 32 \mathrm{ft} E$ of Col. 19 \\
\hline VT695 & $19-20 \mathrm{~W}$ & $<0.05 *$ & $<0.05$ & Floor & Floor, $9.5 \mathrm{ft} \mathrm{S} \mathrm{\&} 32 \mathrm{ft} \mathrm{E}$ of Col. 20 \\
\hline VT696 & $20-21 W$ & $<0.05 *$ & $<0.05$ & Floor & Floor, $9.5 \mathrm{ft} \mathrm{S} \mathrm{\&} 32 \mathrm{ft} E$ of Col. 21 \\
\hline VT697 & $8-9 \mathrm{E}$ & $<0.05 *$ & $<0.05$ & Floor & Floor, $9.5 \mathrm{ft} \mathrm{S} \& 10 \mathrm{ft} \mathrm{W}$ of Col. 9 \\
\hline VT698 & $20-21 E$ & $<0.05 *$ & $<0.10$ & Window & $\begin{array}{l}\text { Wooden window ledge (middle window) in middle of } \\
\text { bay }\end{array}$ \\
\hline VT699 & 19-20E & $<0.05^{*}$ & $<0.10$ & Window & $\begin{array}{l}\text { Wooden window ledge (middle window) in middle of } \\
\text { bay }\end{array}$ \\
\hline VT700 & $18-19 E$ & $<0.05 *$ & $<0.10$ & Window & $\begin{array}{l}\text { Wooden window ledge (middle window) in middle of } \\
\text { bay }\end{array}$ \\
\hline VT701 & $17-18 \mathrm{E}$ & 0.37 * & 0.74 & Window & $\begin{array}{l}\text { Wooden window ledge (middle window) in middle of } \\
\text { bay }\end{array}$ \\
\hline VT702 & $16-17 \mathrm{E}$ & $<0.05^{*}$ & $<0.10$ & Window & $\begin{array}{l}\text { Wooden window ledge (middle window) in middle of } \\
\text { bay }\end{array}$ \\
\hline VT703 & $15-16 \mathrm{E}$ & $<0.05 *$ & $<0.10$ & Window & $\begin{array}{l}\text { Wooden window ledge (middle window) in middle of } \\
\text { bay }\end{array}$ \\
\hline
\end{tabular}


Table 4. (continued)

\begin{tabular}{|c|c|c|c|c|c|}
\hline $\begin{array}{l}\text { Sample } \\
\text { ID } a\end{array}$ & $\begin{array}{c}\text { Bay/room/ } \\
\text { structure/ } \\
\text { area }^{b}\end{array}$ & $\begin{array}{c}\mathrm{Be}^{c} \\
(\mu \mathrm{g} / \mathrm{g}) \\
(\mu \mathrm{g})^{*}\end{array}$ & $\begin{array}{c}\text { Be/area } \\
\left(\mu \mathrm{g} / \mathrm{ft}^{2}\right)^{d}\end{array}$ & $\begin{array}{l}\text { Location } \\
\text { in bay }\end{array}$ & Location notese \\
\hline VT704 & $14-15 E$ & $<0.05 *$ & $<0.10$ & Window & $\begin{array}{l}\text { Wooden window ledge (middle window) in middle of } \\
\text { bay }\end{array}$ \\
\hline VT705 & $13-14 \mathrm{E}$ & $<0.05^{*}$ & $<0.10$ & Window & $\begin{array}{l}\text { Wooden window ledge (middle window) in middle of } \\
\text { bay }\end{array}$ \\
\hline VT706 & $12-13 E$ & $<0.05^{*}$ & $<0.10$ & Window & $\begin{array}{l}\text { Wooden window ledge (middle window) in middle of } \\
\text { bay }\end{array}$ \\
\hline VT707 & $11-12 E$ & $<0.05 *$ & $<0.10$ & Window & Wooden window ledge, only window in bay \\
\hline VT708 & 10-11E & $<0.05 *$ & $<0.10$ & Window & $\begin{array}{l}\text { Wooden window ledge (middle window) in middle of } \\
\text { bay }\end{array}$ \\
\hline VT709 & $9-10 \mathrm{E}$ & $<0.05^{*}$ & $<0.10$ & Window & $\begin{array}{l}\text { Wooden window ledge (middle window) in middle of } \\
\text { bay }\end{array}$ \\
\hline VT710 & $6-7 S$ & $<0.05 *$ & $<0.10$ & Wall & Top of block wall in middle of bay \\
\hline VT711 & $6-7 S$ & $<0.05^{*}$ & $<0.10$ & Bridging & Bridging (E) 23 way up towards peak \\
\hline VT712 & $5-6 S$ & $<0.05^{*}$ & $<0.10$ & Wall & Top of block wall in middle of bay \\
\hline VT713 & $5-6 S$ & $<0.05 *$ & $<0.10$ & Bridging & Bridging $(E), 2 \backslash 3$ of the way up towards peak \\
\hline VT714 & $5 S$ & $<0.05^{*}$ & $<0.10$ & Truss & Top of Truss 512 of the way up towards peak \\
\hline VT715 & $4-5 S$ & $<0.05 *$ & $<0.10$ & Wall & Top of block wall in middle of bay \\
\hline VT716 & $4-5 S$ & $<0.05^{*}$ & $<0.10$ & Window & Wooden window ledge on $E$ window \\
\hline VT717 & $5-6 S$ & $<0.05 *$ & $<0.10$ & Window & Wooden window ledge of middle window \\
\hline VT718 & $4-5 S$ & $<0.05^{*}$ & $<0.10$ & Bridging & Bridging $(E), 13$ of the way up towards the peak \\
\hline VT719 & $4 S$ & $<0.05 *$ & $<0.10$ & Truss & Top of Truss 4), $1 \backslash 3$ of the way up towards peak \\
\hline VT720 & $3-4 S$ & $<0.05 *$ & $<0.10$ & Window & Wooden window ledge of the middle window \\
\hline VT721 & $18 \mathrm{~W}-19 \mathrm{~W}$ & $<0.05^{*}$ & $<0.10$ & Window & Window ledge (wood) between $18 \mathrm{~W} \& 19 \mathrm{~W}$ \\
\hline VT722 & $19 W-20 W$ & $<0.05^{*}$ & $<0.10$ & Window & Window ledge (wood) between $19 \mathrm{~W} \& 20 \mathrm{~W}$ \\
\hline VT723 & $20 \mathrm{~W}-21 \mathrm{~W}$ & $<0.05 *$ & $<0.10$ & Window & Window ledge (wood) between $20 \mathrm{~W} \& 21 \mathrm{~W}$ \\
\hline VT724 & $6 S-7 S$ & $<0.05^{*}$ & $<0.10$ & Window & Window ledge (wood) between $6 S \& 7 S$ \\
\hline VT725 & $7 \mathrm{~S}-8 \mathrm{E}$ & $<0.05^{*}$ & $<0.10$ & $\begin{array}{l}\text { Window } \\
\text { ledge }\end{array}$ & $\begin{array}{l}\text { Window ledge (wood) between } 7 \mathrm{~S} \& 8 \mathrm{E} \text {, about } 12 \mathrm{ft} \\
\text { east of } 7 \mathrm{~S} \text { (in south triangle) }\end{array}$ \\
\hline VT726 & $7 S-8 E$ & $<0.05 *$ & $<0.10$ & $\begin{array}{l}\text { Window } \\
\text { ledge }\end{array}$ & $\begin{array}{l}\text { Window ledge (wood) between } 7 \mathrm{~S} \& 8 \mathrm{E} \text { about } 36 \mathrm{ft} \\
\text { east of } 7 \mathrm{~S} \text { (in south triangle) }\end{array}$ \\
\hline VT727 & $7 \mathrm{~S}-8 \mathrm{E}$ & $<0.05^{*}$ & $<0.10$ & $\begin{array}{l}\text { Window } \\
\text { ledge }\end{array}$ & $\begin{array}{l}\text { Window ledge (wood) between } 7 \mathrm{~S} \& 8 \mathrm{E} \text { about } 12 \mathrm{ft} \\
\text { west of } 8 \mathrm{E} \text { (in south triangle) }\end{array}$ \\
\hline VT728 & $8 \mathrm{E}-9 \mathrm{E}$ & $<0.05 *$ & $<0.10$ & Window & Window ledge (wood) between $8 \mathrm{E} \& 9 \mathrm{E}$ \\
\hline VT729 & $1 N$ & $0.08 *$ & 0.16 & Wall & $\begin{array}{l}\text { Top of block wall (wood plate), W wall of wood shop, } \\
\text { about } 14 \mathrm{ft} S \text { of NW comer }\end{array}$ \\
\hline
\end{tabular}


Table 4. (continued)

\begin{tabular}{|c|c|c|c|c|c|}
\hline $\begin{array}{l}\text { Sample } \\
\text { ID } a\end{array}$ & $\begin{array}{l}\text { Bay/room/ } \\
\text { structure/ } \\
\text { area }^{b}\end{array}$ & $\begin{array}{c}\mathrm{Be}^{c} \\
(\mu \mathrm{g} / \mathrm{g}) \\
(\mu \mathrm{g})^{*}\end{array}$ & $\begin{array}{c}\text { Be/area } \\
\left(\mu \mathrm{g} / \mathrm{ft}^{2}\right)^{d}\end{array}$ & $\begin{array}{l}\text { Location } \\
\text { in bay }\end{array}$ & Location notese \\
\hline VT730 & $1 \mathrm{~N}-2 \mathrm{~N}$ & $0.06 *$ & 0.12 & Window & $\begin{array}{l}\text { Wooden window ledge between Col. 1N \& } 2 \mathrm{~N} \text { (about } \\
4 \mathrm{ft} \text { W of } 2 \mathrm{~N} \text { ) }\end{array}$ \\
\hline VT731 & $1 \mathrm{~N}-2 \mathrm{~N}$ & $0.08 *$ & 0.16 & Wall & Top of block wall (block) between Col. 1N \& $2 \mathrm{~N}$ \\
\hline VT732 & $1 \mathrm{~N}$ & $0.08 *$ & 0.16 & Wall & $\begin{array}{l}\text { Top of block wall (wood plate), W wall of wood shop, } \\
\text { about } 28 \mathrm{ft} S \text { of NW comer }\end{array}$ \\
\hline VT733 & $1 \mathrm{~N}-2 \mathrm{~N}$ & $0.08 *$ & 0.16 & Bridging & Bridging, between $1 \mathrm{~N} \& 2 \mathrm{~N}$ about $2 \backslash 3$ way to peak \\
\hline VT734 & $1 \mathrm{~N}$ & $0.08 *$ & 0.16 & Truss & $\begin{array}{l}\text { Top of truss, about } 2 \backslash 3 \text { way to peak from Col. } 1 \mathrm{~N} \\
\text { (NW corner of wood shop) }\end{array}$ \\
\hline VT735 & $2 \mathrm{~N}$ & $0.08 *$ & 0.16 & Truss & Top of truss, about $2 \backslash 3$ way to peak from $2 N$ \\
\hline VT736 & $2 \mathrm{~N}-3 \mathrm{~N}$ & $0.08 *$ & 0.16 & Bridging & Bridging, about 23 way to peak between $2 N \& 3 N$ \\
\hline VT737 & $2 \mathrm{~N}-3 \mathrm{~N}$ & $0.06 *$ & 0.12 & Window & Wooden window ledge between $2 \mathrm{~N} \& 3 \mathrm{~N}$ \\
\hline VT738 & $2 N-3 N$ & $0.09 *$ & 0.18 & Wall & Top of block wall (wood plate) between $2 \mathrm{~N} \& 3 \mathrm{~N}$ \\
\hline VT739 & $3 N-4 N$ & $0.18 *$ & 0.36 & Wall & Top of block wall (wood plate) between $3 N \& 4 N$ \\
\hline VT740 & $3 N-4 N$ & $0.07 *$ & 0.14 & Window & Wooden window ledge between $3 N \& 4 N$ \\
\hline VT741 & $3 \mathrm{~N}$ & $0.08 *$ & 0.16 & Truss & Top of truss, about 112 way to peak from $3 \mathrm{~N}$ \\
\hline VT742 & $3 N-4 N$ & $0.09 *$ & 0.09 & Stringer & $\begin{array}{l}\text { Stringer (upper vertical surface) about } 12 \text { way to the } \\
\text { peak between } 3 N \& 4 N\end{array}$ \\
\hline VT743 & $3 N-4 N$ & $0.09 *$ & 0.18 & Bridging & Bridging about 112 way to the peak between $3 N \& 4 N$ \\
\hline VT744 & $4 N$ & $0.08 *$ & 0.16 & Truss & Top of truss about 13 way to peak from $4 \mathrm{~N}$ \\
\hline VT745 & $4 N-5 N$ & $0.21 *$ & 0.42 & $\begin{array}{l}\text { Window } \\
\text { ledge }\end{array}$ & $\begin{array}{l}\text { Concrete window ledge (about } 1 \mathrm{ft} \& 8 \text { in. below } \\
\text { window) between } 4 N \& 5 N\end{array}$ \\
\hline VT746 & $4 N-5 N$ & $<0.05 *$ & $<0.10$ & Wall & Top of concrete block wall between $4 \mathrm{~N} \& 5 \mathrm{~N}$ \\
\hline VT747 & $4 \mathrm{~N}-5 \mathrm{~N}$ & $<0.05 *$ & $<0.10$ & Bridging & Bridging, about 13 way to peak between $4 N \& 5 N$ \\
\hline VT748 & $5 \mathrm{~N}$ & $<0.05 *$ & $<0.10$ & Truss & Top of truss about 13 way to peak from $5 \mathrm{~N}$ \\
\hline VT749 & $5 \mathrm{~N}-6 \mathrm{~N}$ & $<0.05 *$ & $<0.10$ & Window & Wooden window ledge between $5 N$ \& $6 \mathrm{~N}$ \\
\hline VT750 & $5 N-6 N$ & $<0.05 *$ & $<0.10$ & Wall & Top of block wall (wood) between $5 \mathrm{~N} \& 6 \mathrm{~N}$ \\
\hline VT751 & $3-4 S$ & $<0.05 *$ & $<0.10$ & Bridging & Bridging, 113 of the way up towards the peak \\
\hline VT752 & $3 S$ & $<0.05 *$ & $<0.10$ & Truss & Top of Truss $3,2 \backslash 3$ way up towards peak \\
\hline VT753 & $3-4 S$ & $<0.05 *$ & $<0.05$ & Stringer & Stringer, top of peak, one stringer from peak \\
\hline VT754 & $3-4 S$ & $<0.05 *$ & $<0.10$ & Wall & Top of block wall in middle of bay \\
\hline VT755 & $2 S-3 S$ & $<0.05 *$ & $<0.10$ & $\begin{array}{l}\text { Window } \\
\text { ledge }\end{array}$ & $\begin{array}{l}\text { Concrete window ledge (about } 1 \mathrm{ft} \& 8 \text { in. below } \\
\text { window) between } 2 S \& 3 S\end{array}$ \\
\hline VT756 & $2 S-3 S$ & $<0.05 *$ & $<0.10$ & Wall & Top of block wall (concrete) between $2 S \& 3 S$ \\
\hline
\end{tabular}


Table 4. (continued)

\begin{tabular}{|c|c|c|c|c|c|}
\hline $\begin{array}{c}\text { Sample } \\
\mathrm{ID}^{a}\end{array}$ & $\begin{array}{c}\text { Bay/room/ } \\
\text { structure/ } \\
\text { area }^{b}\end{array}$ & $\begin{array}{c}\mathrm{Be}^{c} \\
(\mu \mathrm{g} / \mathrm{g}) \\
(\mu \mathrm{g})^{*} \\
\end{array}$ & $\begin{array}{c}\text { Be/area } \\
\left(\mu \mathrm{g} / \mathrm{ft}^{2}\right)^{d} \\
\end{array}$ & $\begin{array}{l}\text { Location } \\
\text { in bay }\end{array}$ & Location notese \\
\hline VT757 & $2 S-3 S$ & $<0.05 *$ & $<0.10$ & Bridging & Bridging, about $2 \backslash 3$ way to peak between $2 S \& 3 S$ \\
\hline VT758 & $2 S$ & $<0.05 *$ & $<0.10$ & $\begin{array}{l}\text { Window } \\
\text { ledge }\end{array}$ & $\begin{array}{l}\text { Wooden window ledge between } 1 S \& 2 S \text { (SW comer } \\
\text { of wood shop) }\end{array}$ \\
\hline VT759 & $1 S-2 S$ & $<0.05 *$ & $<0.10$ & Wall & Top of block wall (concrete) between is \& $2 S$ \\
\hline VT760 & $1 S-2 S$ & $<0.05 *$ & $<0.10$ & Bridging & Bridging, about $1 \mathrm{~K}$ way to peak between $1 \mathrm{~S} \& 2 \mathrm{~S}$ \\
\hline VT761 & $1 S-2 S$ & $<0.05 *$ & $<0.05$ & Stringer & Stringer, about 14 way to peak between $1 S \& 2 S$ \\
\hline VT762 & $1 \mathrm{~S}$ & $0.09 *$ & 0.18 & Truss & $\begin{array}{l}\text { Top of truss about } 14 \text { way to peak from Col. } 1 \mathrm{~S} \text { (SW } \\
\text { corner of wood shop) }\end{array}$ \\
\hline VT763 & 1S & $<0.05 *$ & $<0.10$ & Wall & Top of block wall about $25 \mathrm{ft} \mathrm{N}$ of Col. $1 \mathrm{~S}$ \\
\hline VT764 & $2 S$ & $<0.05^{*}$ & $<0.10$ & Truss & Top of truss about 12 way to peak from Col. $2 \mathrm{~S}$ \\
\hline VT765 & $5 \mathrm{~N}-6 \mathrm{~N}$ & $<0.05 *$ & $<0.10$ & Bridging & Bridging about 233 way to peak between $5 N \& 6 N$ \\
\hline VT766 & $12-13 W$ & $<0.05 *$ & $<0.05$ & Stringer & Stringer, $2 \backslash 3$ of the way up towards the peak \\
\hline VT767 & 19-20E & $<0.05 *$ & $<0.05$ & Stringer & Stringer, $2 \backslash 3$ of the way up towards the peak \\
\hline VT768 & $1-2 \mathrm{~N}$ & $<0.05 *$ & $<0.05$ & Floor & Floor, $9.25 \mathrm{ft} \mathrm{E} \mathrm{\&} 10 \mathrm{ft} \mathrm{S}$ of Col. 1 \\
\hline VT769 & $2-3 N$ & $<0.05 *$ & $<0.05$ & Floor & Floor, $9.5 \mathrm{ft} E \& 10 \mathrm{ft} S$ of Col. 2 \\
\hline VT770 & $3-4 \mathrm{~N}$ & $<0.05 *$ & $<0.05$ & Floor & Floor, $9.5 \mathrm{ft} \mathrm{E} \& 10 \mathrm{ft} \mathrm{S}$ of Col. 3 \\
\hline VT771. & $4-5 \mathrm{~N}$ & $<0.05 *$ & $<0.05$ & Floor & Floor, $9.5 \mathrm{ft}$ E \& $10 \mathrm{ft} S$ of Col. 4 \\
\hline VT772 & $5-6 \mathrm{~N}$ & $<0.05 *$ & $<0.05$ & Floor & Floor, $9.5 \mathrm{ft} \mathrm{E} \mathrm{\&} 10 \mathrm{ft} \mathrm{S}$ of Col. 5 \\
\hline VT773 & $6-7 \mathrm{~N}$ & $<0.05^{*}$ & $<0.05$ & Floor & Floor, $9.5 \mathrm{ft}$ E \& $10 \mathrm{ft} \mathrm{S}$ of Col. 6 \\
\hline VT774 & $7-12 \mathrm{~N}$ & $<0.05 *$ & $<0.05$ & Floor & $\begin{array}{l}\text { Floor, } 9.5 \mathrm{ft} \mathrm{E} \mathrm{\&} 10 \mathrm{ft} \mathrm{S} \text { of Col. 7; This area is a pie- } \\
\text { shaped section }\end{array}$ \\
\hline VT775 & $7-12 S$ & $<0.05 *$ & $<0.05$ & Floor & $\begin{array}{l}\text { Floor, middle of the pie-shaped section bay, } 28 \mathrm{ft} \mathrm{N} \text { of } \\
\text { Col. } 7\end{array}$ \\
\hline VT776 & $6-7 S$ & $<0.05^{*}$ & $<0.05$ & Floor & Floor, $9.5 \mathrm{ft} \mathrm{E} \mathrm{\&} 28 \mathrm{ft} \mathrm{N}$ of Col. 6 \\
\hline VT777 & $5-6 S$ & $0.08 *$ & 0.08 & Floor & Floor, $9.5 \mathrm{ft} \mathrm{E} \mathrm{\&} 28 \mathrm{ft} \mathrm{N}$ of Col. 5 \\
\hline VT778 & $4-5 S$ & $<0.05^{*}$ & $<0.05$ & Floor & Floor, $9.5 \mathrm{E} \& 28 \mathrm{ft} \mathrm{N}$ of Col. 4 \\
\hline VT779 & $3-4 S$ & $<0.05 *$ & $<0.05$ & Floor & Floor, $9.5 \mathrm{ft}$ E \& $28 \mathrm{ft} \mathrm{N}$ of Col. 3 \\
\hline VT780 & $2-3 S$ & $<0.05 *$ & $<0.05$ & Floor & Floor, $9.5 \mathrm{ft} \mathrm{E} \mathrm{\&} 28 \mathrm{ft} \mathrm{N}$ of Col. 2 \\
\hline VT781 & $1-2 S$ & $<0.05 *$ & $<0.05$ & Floor & Floor, $9.25 \mathrm{ft} \mathrm{E} \mathrm{\&} 28 \mathrm{ft} \mathrm{N}$-of Col. 1 \\
\hline VT782 & $41-42 W$ & $0.08 *$ & 0.16 & Bridging & $\begin{array}{l}\text { Bridging (taken before add. recleaning) room } N \text { of } \\
\text { refrig.rm. \& } 10 \mathrm{ft} \mathrm{S} \& \mathrm{~W} \text { of Col. } 42 \text {. }\end{array}$ \\
\hline VT783 & MS-4 & $<0.05 *$ & $<0.10$ & Bridging & $\begin{array}{l}\text { Bridging, Bay } 39-40 \mathrm{~W} \text {, refrigeration room in mid. side } \\
\text { of bay, MS- } 3 \text { middle of the bay, MS- } 4\end{array}$ \\
\hline
\end{tabular}


Table 4. (continued)

\begin{tabular}{|c|c|c|c|c|c|}
\hline $\begin{array}{l}\text { Sample } \\
\text { ID } a\end{array}$ & $\begin{array}{c}\text { Bay/room/ } \\
\text { structure/ } \\
\text { area }^{b}\end{array}$ & $\begin{array}{c}\mathrm{Be}^{c} \\
(\mu \mathrm{g} / \mathrm{g}) \\
(\mu \mathrm{g})^{*}\end{array}$ & $\begin{array}{l}\text { Be/area } \\
\left(\mu \mathrm{g} / \mathrm{ft}^{2}\right)^{d}\end{array}$ & $\begin{array}{l}\text { Location } \\
\text { in bay }\end{array}$ & Location notese \\
\hline VT784 & MS-4 & $0.20 *$ & 0.40 & Bridging & $\begin{array}{l}\text { Bridging, Bay } 37-38 \mathrm{~W} \text {, refrigeration room in middle of } \\
\text { the bay, MS-4 }\end{array}$ \\
\hline VT785 & MS-3 & $<0.05 *$ & $<0.10$ & Bridging & $\begin{array}{l}\text { Bridging, Bay } 36-37 \mathrm{~W} \text {, refrigeration room on } \mathrm{S} \text { side } \\
\text { bay }\end{array}$ \\
\hline VT786 & MS-1 & $0.09 *$ & 0.18 & Bridging & $\begin{array}{l}\text { Bridging, Bay } 24-25 \mathrm{~W} \text {, electrical room about } 10 \mathrm{ft} \mathrm{W} \\
\& 10 \mathrm{ft} \text { S of Col } 25 \mathrm{~W}, \mathrm{MS}-1\end{array}$ \\
\hline VT787 & MS-1 & $<0.05 *$ & $<0.10$ & Bridging & $\begin{array}{l}\text { Bridging, bay } 24-25 \mathrm{~W} \text {,electrical room about } 20 \mathrm{ft} \mathrm{W} \\
\& 20 \mathrm{ft} \mathrm{S} \text { of Col. } 25 \mathrm{~W}, \mathrm{MS}-1\end{array}$ \\
\hline VT788 & MS-1 & $0.26 *$ & 0.52 & Bridging & $\begin{array}{l}\text { Bridging, Bay } 23-24 \mathrm{~W} \text {, electrical room about } 5 \mathrm{ft} 2 \mathrm{ft} \\
\mathrm{W} \& 10 \mathrm{ft} \mathrm{S} \text { of Col. } 24 \mathrm{~W}, \mathrm{MS}-1\end{array}$ \\
\hline VT790 & Drain 42 & $0.27 *$ & 0.54 & Drain & $\begin{array}{l}\text { Sanitary drain pipe (running } N \text { to } S \text { of bldg.) at } 2 \mathrm{ft} \& \\
1 \text { in. E of Col. } 27 \mathrm{~W}\end{array}$ \\
\hline VT791 & Refrig. rm. & $0.33 *$ & 0.66 & Floor & Floor in refrigeration room over old VM449 location \\
\hline VT792 & Refrig. rm. & $0.05 *$ & 0.20 & Floor & Floor in refrigeration room over old VM451 location \\
\hline VT793 & Refrig. rm. & $0.42 *$ & 0.84 & Floor & Floor in refrigeration room over old VM451 location \\
\hline VT794 & Refrig. rm. & $<0.05 *$ & $<0.10$ & Floor & Floor in refrigeration room over old VM452 location \\
\hline VT795 & Refrig. rm. & $0.34 *$ & 0.68 & Floor & Floor in refrigeration room over old VM453 location \\
\hline VT796 & $38 \mathrm{~W}$ & $0.21 *$ & 0.21 & Floor & $\begin{array}{l}\text { Floor in same location as previous sample (VM464) } \\
\text { which is near Col. } 38 \mathrm{~W}\end{array}$ \\
\hline VT797 & $38 \mathrm{~W}$ & $0.19 *$ & 0.19 & Floor & $\begin{array}{l}\text { Smear was taken at the same location of the previous } \\
\text { sample (VM465) near Col. } 38 \mathrm{~W}\end{array}$ \\
\hline VT798 & $39 \mathrm{~W}$ & $0.83 *$ & 0.83 & Floor & $\begin{array}{l}\text { Floor smear at same location of previous sample } \\
\text { (VM466) in ref. rm. near Col. } 39 \mathrm{~W}\end{array}$ \\
\hline VT799 & 26-27E & $0.10 *$ & 0.10 & Stringer & $\begin{array}{l}\text { Upper side of stringer between } 26 \mathrm{E} \& 27 \mathrm{E} \text { after } \\
\text { addition. cleaning performed }\end{array}$ \\
\hline VT801 & 20-21E & $0.08 *$ & 0.16 & Wall & $\begin{array}{l}\text { Top of block wall (wood plate) between } 20 \mathrm{E} \& 21 \mathrm{E} \\
\text { after add. clean. near old VT567 }\end{array}$ \\
\hline VT803 & $25 \mathrm{E}$ & $0.10 *$ & 0.10 & Other & $\begin{array}{l}\text { Ceiling "I" beam (previously smeared for } \mathrm{Be} \text { ) at } 6.5 \mathrm{ft} \\
\mathrm{N} \text { and } 13 \mathrm{ft} \mathrm{E} \text { of Col. } 25 \mathrm{E}\end{array}$ \\
\hline VT804 & $24 \mathrm{E}$ & $0.12 *$ & 0.12 & Other & $\begin{array}{l}\text { Ceiling "I" beam (previously smeared) at } 9.5 \mathrm{ft} N \& 2 \\
\mathrm{ft} E \text { of Col. } 24 \mathrm{E}\end{array}$ \\
\hline VT805 & $24 \mathrm{E}$ & $0.11 *$ & 0.11 & Other & Ceiling "I" beam at $2.33 \mathrm{ft} \mathrm{S} \mathrm{\&} 13 \mathrm{ft} \mathrm{E}$ of Col. 24E \\
\hline VT806 & $24 \mathrm{E}$ & $0.13 *$ & 0.13 & Other & Ceiling "I" beam at $13.5 \mathrm{ft} \mathrm{S} \& 2 \mathrm{ft} E$ of Col.24E \\
\hline VT807 & $28-29 \mathrm{E}$ & $1.3^{*}$ & 1.3 & $\begin{array}{l}\text { Window } \\
\text { ledge }\end{array}$ & $\begin{array}{l}\text { Concrete window ledge (additional cleaning after old } \\
\text { VT455) between Col. } 28 E \text { \& } 29 E\end{array}$ \\
\hline
\end{tabular}


Table 4. (continued)

\begin{tabular}{|c|c|c|c|c|c|}
\hline $\begin{array}{c}\text { Sample } \\
\text { ID } a\end{array}$ & $\begin{array}{c}\text { Bay/room/ } \\
\text { structure/ } \\
\text { area }^{b}\end{array}$ & $\begin{array}{c}\mathrm{Bec} \\
(\mu \mathrm{g} / \mathrm{g}) \\
(\mu \mathrm{g})^{*}\end{array}$ & $\begin{array}{c}\text { Be/area } \\
\left(\mu \mathrm{g} / \mathrm{ft}^{2}\right)^{d} \\
\end{array}$ & $\begin{array}{l}\text { Location } \\
\text { in bay }\end{array}$ & Location notes ${ }^{e}$ \\
\hline VT808 & 26-27E & $1.3^{*}$ & 1.3 & $\begin{array}{l}\text { Window } \\
\text { ledge }\end{array}$ & $\begin{array}{l}\text { Concrete window ledge (additional cleaning after old } \\
\text { VT459) between Col. } 26 \mathrm{E} \& 27 \mathrm{E}\end{array}$ \\
\hline VT809 & $2 \mathrm{~N}$ & $0.09 *$ & 0.09 & Other & $\begin{array}{l}\text { Light fixture, } \sim 12 \mathrm{ft} S \text { of Col. } 2 \mathrm{~N} \text { same location as } \\
\text { old VM } 448 \text { after add. cleaning. }\end{array}$ \\
\hline VT810 & $5 S$ & $0.09 *$ & 0.09 & Other & Light fixture about 12 way to peak from Col. $5 \mathrm{~S}$ \\
\hline VT811 & $11 \mathrm{~W}$ & $0.09 *$ & 0.09 & Other & $\begin{array}{l}\text { Light fixture (additional cleaning after old VM447) } \\
\text { about } 14 \text { way to peak from } 11 \mathrm{~W}\end{array}$ \\
\hline VT812 & $15 \mathrm{E}$ & $0.09 *$ & 0.09 & Other & Light fixture about $8 \mathrm{ft} \mathrm{E}$ of peak at Col. line $15 \mathrm{E}$ \\
\hline VT813 & $18 W$ & $0.08 *$ & 0.08 & Other & Light fixture about $6 \mathrm{ft} \mathrm{W}$ of peak on Col. line $18 \mathrm{~W}$ \\
\hline VT814 & $20 \mathrm{E}$ & $0.09 *$ & 0.09 & Other & Light fixture about 13 way to peak from Col. $20 \mathrm{E}$ \\
\hline VT815 & $42 W$ & $0.08 *$ & 0.08 & Other & Light fixture about $10 \mathrm{ft} \mathrm{E}$ of Col. $42 \mathrm{~W}$ \\
\hline VT816 & $39 \mathrm{E}$ & $0.19 *$ & 0.19 & Other & Light fixture about $17 \mathrm{ft}$ W of Col. $39 \mathrm{E}$ \\
\hline VT817 & $36 \mathrm{~W}$ & $0.11 *$ & 0.11 & Other & Light fixture about $16 \mathrm{ft} \mathrm{E}$ of Col. $36 \mathrm{~W}$ \\
\hline VT818 & $32 \mathrm{E}$ & $0.25 *$ & 0.25 & Other & Light fixture about $20 \mathrm{ft} \mathrm{W}$ of Col. $32 \mathrm{E}$ \\
\hline VT819 & $29 W$ & $0.45 *$ & 0.45 & Other & Light fixture about $22 \mathrm{ft}$ E of Col. $29 \mathrm{~W}$ \\
\hline VT820 & $26 \mathrm{E}$ & $0.30 *$ & 0.30 & Other & Light fixture about $12 \mathrm{ft} W$ of Col. $26 \mathrm{E}$ \\
\hline VT821 & $23 \mathrm{~W}$ & $0.13 *$ & 0.13 & Other & Light fixture about $25 \mathrm{ft} \mathrm{E}$ of $\mathrm{Col} .23 \mathrm{~W}$ \\
\hline VT823 & $27 \mathrm{~W}$ & $0.24 *$ & 0.24 & Wall & Boiler room sand box wall (same wall as VM456) \\
\hline VT825 & $27 \mathrm{~W}$ & $0.17 *$ & 0.17 & Wall & $\begin{array}{l}\text { Boiler room sand box wall } 15 \mathrm{ft} \text { due } \mathrm{N} \text { of Col. } \mathrm{ft} \text { due } \\
\mathrm{N} \text { of Col. } 27 \mathrm{~W}\end{array}$ \\
\hline VT827 & $27 \mathrm{~W}$ & $0.24 *$ & 0.24 & Wall & $\begin{array}{l}\text { North wall of boiler room sand box (same wall as } \\
\text { VM455) near Col. } 27 \mathrm{~W}\end{array}$ \\
\hline VT829 & $27 \mathrm{~W}$ & $0.33 *$ & 0.33 & Wall & Boiler room sand box north wall near Col $27 \mathrm{~W}$ \\
\hline VT830 & $27 \mathrm{~W}$ & $0.24 *$ & 0.24 & Wall & $\begin{array}{l}\text { Boiler room sand box west wall (same wall as } \\
\text { VM454) near Col. } 27 \mathrm{~W}\end{array}$ \\
\hline VT831 & $27 \mathrm{~W}$ & $0.21 *$ & 0.21 & Wall & $\begin{array}{l}\text { Boiler room sand box west wall at } 10.5 \mathrm{ft} \mathrm{N} \text { and } 19 \mathrm{ft} \\
\& 1 \text { in. W of Col. } 27 \mathrm{~W} \text {. }\end{array}$ \\
\hline VT863 & Drain & $0.40 *$ & 0.80 & Drain & $\begin{array}{l}\text { Horizontal cast iron drain pipe } 38 \mathrm{ft} \mathrm{N} \mathrm{\&} 12 \mathrm{ft} \mathrm{W} \text { of } \\
\text { Col. 2S; a VT } 840 \text { resmear }\end{array}$ \\
\hline VT864 & Drain 13 & $0.40 *$ & 1.6 & Drain & $\begin{array}{l}\text { Cast iron drain pipe at } 21 \mathrm{ft} \mathrm{S} \mathrm{\&} 10 \mathrm{ft} \mathrm{W} \text { of Col. } 2 \mathrm{~N} \text {; } \\
\text { a resmear of VT } 842\end{array}$ \\
\hline VT865 & Drain 57 & $0.40 *$ & 0.80 & Drain & $\begin{array}{l}\text { Cast iron drain } 57 \text { pipe at } 33 \mathrm{ft} S \& 6 \mathrm{ft} \text { E of Col. } \\
5 \mathrm{~N} \text {; supplements VM481 }\end{array}$ \\
\hline VT867 & Drain 55 & $0.40 *$ & 0.80 & Drain & $\begin{array}{l}\text { Drain } 55 \text { pipe at } 33 \mathrm{ft}, \mathrm{S} \& 7 \mathrm{ft} \mathrm{E} \text { of } \mathrm{Col} 5 \mathrm{~N} \text {; a } \\
\text { resmear of VT } 850\end{array}$ \\
\hline
\end{tabular}


Table 4. (continued)

\begin{tabular}{|c|c|c|c|c|c|}
\hline $\begin{array}{c}\text { Sample } \\
\text { ID } a\end{array}$ & $\begin{array}{c}\text { Bay/room/ } \\
\text { structure/ } \\
\text { area }^{b} \\
\end{array}$ & $\begin{array}{c}\mathrm{Be}^{\mathrm{c}} \\
(\mu \mathrm{g} / \mathrm{g}) \\
(\mu \mathrm{g})^{*} \\
\end{array}$ & $\begin{array}{c}\text { Be/area } \\
\left(\mu \mathrm{g} / \mathrm{ft}^{2}\right)^{d}\end{array}$ & $\begin{array}{l}\text { Location } \\
\text { in bay }\end{array}$ & Location notese \\
\hline VT868 & Drain 54 & 0.40 * & 0.80 & Drain & $\begin{array}{l}\text { Cast iron drain } 54 \text { pipe at } 32 \mathrm{ft} \mathrm{S} \mathrm{\&} 7 \mathrm{ft} \mathrm{E} \text { from Col. } \\
28 \mathrm{E} \text {; a resmear of VT508 }\end{array}$ \\
\hline VT870 & Drain 39 & $0.64 *$ & 1.3 & Drain & $\begin{array}{l}\text { Clay drain pipe at } 31 \mathrm{ft} \& 5 \text { in. W, and } 8 \mathrm{ft} S \text { of Col. } \\
27 \mathrm{E}\end{array}$ \\
\hline VT871 & Drain 64 & $0.40 *$ & 1.6 & Drain & $\begin{array}{l}\text { Cast clay drain pipe at } 28 \mathrm{ft} \& 10 \mathrm{in} . \mathrm{W} \text { and } 8 \mathrm{ft} \mathrm{S} \text { of } \\
\text { Col. } 27 \mathrm{E}\end{array}$ \\
\hline VT872 & Truss & $0.40 *$ & 0.80 & Truss & $\begin{array}{l}\text { Lower truss support about } 1 / 4 \text { way toward peak from } \\
\text { Col. } 28 \mathrm{E} \text {; a resmear of VT508 }\end{array}$ \\
\hline VT873 & $28 E$ & $0.40 *$ & 0.80 & Truss & $\begin{array}{l}\text { Upper truss about } 1 / 4 \text { way to peak from Col. } 28 \mathrm{E} \text {; a } \\
\text { resmear of VT510 }\end{array}$ \\
\hline VT874 & $25-26 \mathrm{~W}$ & $0.40 *$ & 0.80 & Bridging & $\begin{array}{l}\text { Bridging about } 1 / 3 \text { way to peak from between } 25 \mathrm{~W} \& \\
26 \mathrm{~W} \text {; a resmear of VT542 }\end{array}$ \\
\hline VT875 & $24-25 W$ & $0.40 *$ & 0.80 & $\begin{array}{l}\text { Window } \\
\text { ledge }\end{array}$ & $\begin{array}{l}\text { Window ledge between } 24 W \text { \& } 25 \mathrm{~W} \text {; a resmear of } \\
\text { VT519 \& VT } 836\end{array}$ \\
\hline VT876 & $24-25 \mathrm{E}$ & 0.40 * & 0.40 & Wall & $\begin{array}{l}\text { Top of block wall (wood plate) between 24E \& 25E; a } \\
\text { resmear of VT511 \& VT838 }\end{array}$ \\
\hline VT877 & $23-24 \mathrm{~W}$ & $0.40 *$ & 0.80 & Bridging & $\begin{array}{l}\text { Bridging about } 3 / 4 \text { way to peak between } 23 \mathrm{~W} \& 24 \mathrm{~W} \text {; } \\
\text { a resmear of VT550. }\end{array}$ \\
\hline VT878 & $23-24 \mathrm{E}$ & $0.82 *$ & 0.82 & Other & $\begin{array}{l}\text { Top of corner concrete header between } 23 \mathrm{E} \& 24 \mathrm{E} \text {; a } \\
\text { resmear of VT800 }\end{array}$ \\
\hline VT881 & Drain & $0.40 *$ & 0.80 & Drain & $\begin{array}{l}\text { Horizontal drain line opening at } 21 \mathrm{ft} \text { E \& } 7 \mathrm{ft} \mathrm{S} \text { of } \\
\text { Col. } 26 \mathrm{~W} \text {. }\end{array}$ \\
\hline VT885 & $22 W$ & $0.62 *$ & 0.62 & Floor & $\begin{array}{l}\text { Old floor under raised floor at } 1 \mathrm{ft} \mathrm{W} \& 1 \mathrm{ft} S \text { of NE } \\
\text { corner of closet }\end{array}$ \\
\hline VT887 & $22 W$ & $0.38 *$ & 0.38 & Wall & $\begin{array}{l}\text { First (from floor) } \mathrm{N} \text { wall block face at } 7 \mathrm{ft} \text { from NE } \\
\text { corner of old health physics office }\end{array}$ \\
\hline VT889 & $22 W$ & $0.22 *$ & 0.22 & Floor & $\begin{array}{l}\text { Old tile floor (under carpet) in NE corner of old health } \\
\text { physics office (west of Col. } 22 W \text { ) }\end{array}$ \\
\hline VT891 & $22 W$ & $0.43 *$ & 1.7 & Other & $\begin{array}{l}\text { Top of sprinkler system pipe above drop ceiling in } \mathrm{NE} \\
\text { corner of old health physics office }\end{array}$ \\
\hline VT892 & Drain pit & 0.40 * & 0.80 & Drain & $\begin{array}{l}\text { Smear (to } 6 \text { in. depth) clay drain pipe to a pit at } 7.5 \mathrm{ft} \\
S \& 3 \mathrm{ft} E \text { of Col. } 24 \mathrm{~W}\end{array}$ \\
\hline VT894 & Drain pit & 0.40 * & 0.80 & Drain & $\begin{array}{l}\text { Smear (to } 6 \text { in. depth) of a clay drain to a pit at } 7.5 \mathrm{ft} \\
S \& 3 \mathrm{ft} E \text { of Col. } 24 \mathrm{~W}\end{array}$ \\
\hline VT896 & Drain & $0.40 *$ & 1.3 & Drain & $\begin{array}{l}\text { Smear ( } 10 \text { in. deep) of black iron drain pipe at } 2 \mathrm{ft} \mathrm{N} \\
\& 2.25 \mathrm{ft} \mathrm{E} \text { of Col. } 24 \mathrm{~W}\end{array}$ \\
\hline VT900 & Drain 38 & 0.40 * & 0.80 & Drain & Drain pipe smear at $11.5 \mathrm{ft} \mathrm{S} \& 4.5 \mathrm{ft} \mathrm{E}$ of Col. $26 \mathrm{~W}$ \\
\hline
\end{tabular}


Table 4. (continued)

\begin{tabular}{|c|c|c|c|c|c|}
\hline $\begin{array}{l}\text { Sample } \\
\text { ID } a\end{array}$ & $\begin{array}{c}\text { Bay/room/ } \\
\text { structure/ } \\
\text { area }{ }^{b}\end{array}$ & $\begin{array}{c}\mathrm{Be}^{c} \\
(\mu \mathrm{g} / \mathrm{g}) \\
(\mu \mathrm{g})^{*} \\
\end{array}$ & $\begin{array}{c}\text { Be/area } \\
\left(\mu \mathrm{g} / \mathrm{ft}^{2}\right)^{d} \\
\end{array}$ & $\begin{array}{l}\text { Location } \\
\text { in bay }\end{array}$ & Location notese \\
\hline VT902 & Drain 43 & $0.40 *$ & 0.80 & Drain & $\begin{array}{l}\text { Drain pipe smear (to } 6 \text { in. vertical depth) at } 1.5 \mathrm{ft} \mathrm{N} \& \\
2 \mathrm{ft} \text { E of Col. } 28 \mathrm{~W}\end{array}$ \\
\hline VT904 & Drain 1 & $0.40 *$ & 0.80 & Drain & $\begin{array}{l}\text { Drain pipe smear (to vertical depth of } 6 \mathrm{in} \text {.) at } 1 \mathrm{ft} \mathrm{S} \\
\& 20.5 \mathrm{ft} E \text { of Col. } 29 \mathrm{~W}\end{array}$ \\
\hline VT906 & Drain 45 & $0.40 *$ & 0.80 & Drain & $\begin{array}{l}\text { Drain pipe smear (to vertical depth of } 10 \mathrm{in} \text {.) at } 7.5 \mathrm{ft} \\
\mathrm{S} \& 31 \mathrm{ft} \mathrm{E} \text { of } \mathrm{Col} .30 \mathrm{~W}\end{array}$ \\
\hline VT908 & Drain 59 & $0.40 *$ & 0.80 & Drain & $\begin{array}{l}\text { Drain pipe smear (to } 6 \text { in. vertical depth) at } 20.5 \mathrm{ft} \\
\text { east of Col. } 31 \mathrm{~W}\end{array}$ \\
\hline VT911 & Drain 69 & $0.40 *$ & 1.6 & Drain & $\begin{array}{l}\text { Roof drain } 2 \text { (just inside horizontal access) at } 018 \mathrm{ft} \\
\text { due } E \text { of Col. } 37 \mathrm{~W}\end{array}$ \\
\hline VT913 & $2 \mathrm{~N}$ & $0.23 *$ & 0.46 & Other & $\begin{array}{l}\text { Top of steam line (just below ceiling) at } 14 \mathrm{ft} \text { due } \mathrm{N} \\
\text { of Col. } 2 \mathrm{~N} \text { in Room WS-1 }\end{array}$ \\
\hline VT914 & $2 \mathrm{~N}$ & $0.29 *$ & 0.58 & Window & $\begin{array}{l}\text { Window ledge due } \mathrm{N} \text { of } \mathrm{Col} .2 \mathrm{~N} \text { at } \mathrm{N} \text { wall in room } \\
\text { WS-1 }\end{array}$ \\
\hline VT915 & $3 \mathrm{~N}$ & $0.18 *$ & 0.36 & Other & $\begin{array}{l}\text { Sprinkler line (just below ceiling) at } 3 \mathrm{ft} \mathrm{N} \& 2 \mathrm{ft} \mathrm{W} \\
\text { of Col. } 3 \mathrm{~N} \text { in Room WS-2 }\end{array}$ \\
\hline VT916 & $2 \mathrm{~N}$ & $0.26 *$ & 0.26 & Floor & Concrete floor in WS- 1 area at $10 \mathrm{ft}$ due $\mathrm{N}$ of Col. $2 \mathrm{~N}$ \\
\hline VT918 & $3 \mathrm{~N}$ & $0.32 *$ & 0.32 & Floor & Concrete floor in WS-2 area at $10 \mathrm{ft}$ due $\mathrm{N}$ of Col. 3 \\
\hline VT920 & $3 \mathrm{~N}$ & $<0.10^{*}$ & $f$ & Floor & $\begin{array}{l}\text { Concrete floor in WS-3 area (men's bathroom) at } 10 \mathrm{ft} \\
\mathrm{N} \& 10 \mathrm{ft} \mathrm{E} \text { of Col. } 3 \mathrm{~N}\end{array}$ \\
\hline VT922 & $4 \mathrm{~N}$ & $0.18 *$ & 0.18 & Floor & $\begin{array}{l}\text { Concrete floor in WS- } 4 \text { area at } 8 \mathrm{ft} \mathrm{N} \text { and } 6 \mathrm{ft} E \text { of } \\
\text { Col. } 4 \mathrm{~N}\end{array}$ \\
\hline VT924 & $5 \mathrm{~N}$ & $0.31 *$ & 0.31 & Floor & $\begin{array}{l}\text { Concrete floor (WS-5) area at } 7 \mathrm{ft} \mathrm{N} \& 5 \mathrm{ft} \mathrm{E} \text { of Col. } \\
5 \mathrm{~N}\end{array}$ \\
\hline VT925 & $3 \mathrm{~N}$ & $0.51 *$ & 1.0 & $\begin{array}{l}\text { Window } \\
\text { ledge }\end{array}$ & $\begin{array}{l}\text { Visibly very dirty wooden window ledge } 10 \mathrm{ft} E \text { of } \\
\text { Col. } 3 \mathrm{~N} \text { at } \mathrm{N} \text { wall in WS-3 }\end{array}$ \\
\hline VT926 & $4 N$ & $<0.10 *$ & $<0.10$ & Other & $\begin{array}{l}\text { Upper surface of sheetrock (equal to bridging) } 12 \mathrm{ft} \text { due } \\
\mathrm{N} \text { of Col. } 4 \mathrm{~N} \text { in WS }-4\end{array}$ \\
\hline VT927 & $4 \mathrm{~N}$ & $<0.10^{*}$ & $<0.10$ & Wall & $\begin{array}{l}\text { Top of block wall (shower room partition) at } 3 \mathrm{ft} \text { due } \\
\mathrm{N} \text { of Col. } 4 \mathrm{~N} \text { in WS-4 }\end{array}$ \\
\hline VT928 & $5 \mathrm{~N}$ & $<0.10 *$ & $<0.20$ & Other & $\begin{array}{l}\text { Top of steam line (just below ceiling) } 14 \mathrm{ft} \text { due } \mathrm{N} \text { of } \\
\text { Col. } 5 \mathrm{~N} \text { in Room WS-5 }\end{array}$ \\
\hline VT929 & $5 \mathrm{~N}$ & $<0.10^{*}$ & $<0.20$ & $\begin{array}{l}\text { Window } \\
\text { ledge }\end{array}$ & $\begin{array}{l}\text { Wooden window ledge } 6 \mathrm{ft} \mathrm{W} \text { of Col. } 5 \mathrm{~N} \text { at north wall } \\
\text { in room WS- } 5\end{array}$ \\
\hline VT930 & $6 \mathrm{~N}$ & $<0.10 *$ & $<0.10$ & Other & $\begin{array}{l}\text { Top of "I" beam (runs } N \text { to } S \text { ) at } 8 \mathrm{ft} \mathrm{N} \mathrm{\&} 4 \mathrm{ft} \mathrm{W} \text { of } \\
\text { Col. } 6 \mathrm{~N} \text { in Room WS- } 6\end{array}$ \\
\hline VT931 & $6 \mathrm{~N}$ & $<0.10 *$ & $<0.20$ & Other & $\begin{array}{l}\text { Top of sprinkler line at } 10 \mathrm{ft} \mathrm{E} \& 4 \mathrm{ft} \mathrm{N} \text { of Col. } 6 \mathrm{~N} \\
\text { in room WS- } 6\end{array}$ \\
\hline
\end{tabular}


Table 4. (continued)

\begin{tabular}{|c|c|c|c|c|c|}
\hline $\begin{array}{l}\text { Sample } \\
\text { ID } a\end{array}$ & $\begin{array}{c}\text { Bay/room/ } \\
\text { structure/ } \\
\text { area }^{b}\end{array}$ & $\begin{array}{c}\mathrm{Be}^{c} \\
(\mu \mathrm{g} / \mathrm{g}) \\
(\mu \mathrm{g})^{*}\end{array}$ & $\begin{array}{l}\text { Be/area } \\
\left(\mu \mathrm{g} / \mathrm{ft}^{2}\right)^{d}\end{array}$ & $\begin{array}{c}\text { Location } \\
\text { in bay }\end{array}$ & Location notese \\
\hline VT933 & $6 \mathrm{~N}$ & $0.11 *$ & 0.11 & Floor & $\begin{array}{l}\text { Concrete floor (WS-6) area at } 9 \mathrm{ft} \mathrm{N} \& 5 \mathrm{ft} \mathrm{W} \text { of Col. } \\
6 \mathrm{~N} \text {. }\end{array}$ \\
\hline VT935 & $7 \mathrm{~N}$ & $0.12 *$ & 0.12 & Floor & $\begin{array}{l}\text { Tile floor in Allwash tool room of WS-7 area at } 10 \mathrm{ft} \\
\mathrm{N} \& 2 \mathrm{ft} E \text { of Col. } 7 \mathrm{~N}\end{array}$ \\
\hline VT937 & $7 \mathrm{~N}$ & $0.12 *$ & 0.12 & Floor & $\begin{array}{l}\text { Tile floor of Allwash storage rm. (WS-8) entrance at } 5 \\
\mathrm{ft} N \& 10 \mathrm{ft} E \text { of Col. } 7 \mathrm{~N}\end{array}$ \\
\hline VT939 & $7 N$ & $0.10 *$ & 0.10 & Floor & $\begin{array}{l}\text { Carpet area in Allwash storage rm. (WS-8) at } 9 \mathrm{ft} \& 9 \\
\mathrm{ft} E \text { of Col. } 7 \mathrm{~N}\end{array}$ \\
\hline VT941 & $7 \mathrm{~N}$ & $<0.10^{*}$ & $<0.20$ & Other & $\begin{array}{l}\text { Top of sprinkler line at } 16 \mathrm{ft} \mathrm{N} \& 4 \mathrm{ft} \mathrm{W} \text { of Col. } 7 \mathrm{~N} \\
\text { in room WS-7 }\end{array}$ \\
\hline VT942 & $7 N$ & $<0.10^{*}$ & $<0.20$ & Other & $\begin{array}{l}\text { Top of steam line at } 2 \mathrm{ft} \mathrm{N} \& 12 \mathrm{ft} \mathrm{E} \text { of Col. } 7 \mathrm{~N} \text { in } \\
\text { room WS- } 8\end{array}$ \\
\hline VT943 & $13 \mathrm{~W}$ & $0.27 *$ & 0.54 & Other & $\begin{array}{l}\text { Top of steam line at } 4 \mathrm{ft} \mathrm{N} \& 15 \mathrm{ft} \mathrm{W} \text { of Col. } 13 \mathrm{~W} \text { in } \\
\text { room WS-9. }\end{array}$ \\
\hline VT944 & $13 W$ & $0.19 *$ & 0.38 & Other & $\begin{array}{l}\text { Top of steam line at } 15 \mathrm{ft} \mathrm{N} \& 15 \mathrm{ft} \mathrm{W} \text { of Col.13W } \\
\text { in room WS-10 }\end{array}$ \\
\hline VT945 & $13 W$ & $<0.10^{*}$ & $<0.10$ & Floor & $\begin{array}{l}\text { Carpeted surface in KAPL office area (WS-9) at } 4 \mathrm{ft} \mathrm{N} \\
\& 16 \mathrm{ft} \mathrm{W} \text { of Col. } 13 \mathrm{~W}\end{array}$ \\
\hline VT946. & $13 W$ & $<0.10^{*}$ & $<0.10$ & Floor & $\begin{array}{l}\text { Carpeted area of entrance room (WS-10) at } 15 \mathrm{ft} \mathrm{N} \& \\
9 \mathrm{ft} \mathrm{W} \text { of Col. } 13 \mathrm{~W}\end{array}$ \\
\hline VT948 & MS-1 & $0.28 *$ & 0.28 & Stack & Stack floor (center) sampled east of VM515 \\
\hline VT950 & MS-1 & $0.15 *$ & 0.15 & Stack & $\begin{array}{l}\text { Stack south inside wall at } 9.5 \mathrm{ft} \text { above stack floor } \\
\text { level (directly above VM516) }\end{array}$ \\
\hline VT951 & MS-1 & $<0.10^{*}$ & $<0.10$ & Stack & $\begin{array}{l}\text { Stack west inside wall at } 20.5 \mathrm{ft} \text { floor level or } 6 \text { in. } \\
\text { above VM518 }\end{array}$ \\
\hline VT953 & MS-1 & $0.32 *$ & 0.32 & Stack & $\begin{array}{l}\text { Stack north inside wall (very porous red brick surface) } \\
\text { at } 30 \mathrm{ft} \text { from stack floor }\end{array}$ \\
\hline VT955 & MS-1 & $0.14 *$ & 0.14 & Stack & $\begin{array}{l}\text { Stack east inside wall (red brick surface) at } 40 \mathrm{ft} \text { above } \\
\text { stack floor level }\end{array}$ \\
\hline VT956 & MS-1 & $0.18 *$ & 0.18 & Stack & $\begin{array}{l}\text { Stack south inside wall (red brick surface) directly } \\
\text { below VM522 }\end{array}$ \\
\hline VT959 & Drain 49 & $0.10 *$ & 0.20 & Drain & $\begin{array}{l}\text { Drain } 49 \text { (sampled from } 2 \text { in. to } 9.5 \text { in. depth) at } 3 \mathrm{ft} \\
\mathrm{N} \& 5 \mathrm{ft} \mathrm{E} \text { of Col. } 6 \mathrm{~S}\end{array}$ \\
\hline VT960 & Drain 50 & $0.13 *$ & 0.26 & Drain & $\begin{array}{l}\text { Clay pipe drain } 50 \text { (elbow at } 3 \text { in.) at } 7.5 \mathrm{ft} \mathrm{N} \mathrm{\&} 10 \mathrm{ft} \\
\mathrm{E} \text { of Col. } 3 \mathrm{~N}\end{array}$ \\
\hline VT962 & $27 \mathrm{~W}$ & $0.19 *$ & 0.19 & Wall & $\begin{array}{l}\text { Top of west block wall in coal room at } 12 \mathrm{ft} N \text { and } 19 \\
\mathrm{ft} \& 1 \text { in. W of Col. } 27 \mathrm{~W}\end{array}$ \\
\hline
\end{tabular}


Table 4. (continued)

\begin{tabular}{|c|c|c|c|c|c|}
\hline $\begin{array}{c}\text { Sample } \\
\text { ID } a\end{array}$ & $\begin{array}{c}\text { Bay/room/ } \\
\text { structure/ } \\
\text { area } b\end{array}$ & $\begin{array}{c}\mathrm{Be}^{c} \\
(\mu \mathrm{g} / \mathrm{g}) \\
(\mu \mathrm{g})^{*}\end{array}$ & $\begin{array}{l}\text { Be/area } \\
\left(\mu \mathrm{g} / \mathrm{ft}^{2}\right)^{d}\end{array}$ & $\begin{array}{l}\text { Location } \\
\text { in bay }\end{array}$ & Location notese \\
\hline VT964 & $27 \mathrm{~W}$ & $0.12 *$ & 0.24 & Bridging & $\begin{array}{l}\text { Bridging in coal room at } 10.5 \mathrm{ft} \mathrm{N} \& 14 \mathrm{ft} \mathrm{W} \text { of Col. } \\
27 \mathrm{~W}\end{array}$ \\
\hline VT965 & $27 \mathrm{~W}$ & $<0.10^{*}$ & $<0.10$ & Other & $\begin{array}{l}\text { Top of steam line (running N-S) at } 16 \mathrm{ft} \mathrm{N} \& 12.5 \mathrm{ft} \\
W \text { of Col. } 27 \mathrm{~W} \text { in coal room }\end{array}$ \\
\hline VT966 & $27 \mathrm{~W}$ & $0.35 *$ & 0.70 & Bridging & $\begin{array}{l}\text { Bridging in the coal room at } 17 \mathrm{ft} \mathrm{N} \mathrm{\&} 6 \mathrm{ft} \mathrm{W} \text { of Col. } \\
27 \mathrm{~W}\end{array}$ \\
\hline VT967 & $27 \mathrm{~W}$ & $0.29 *$ & 0.29 & Stringer & $\begin{array}{l}\text { Stringer in the coal room at } 14 \mathrm{ft} \mathrm{N} \& 10 \mathrm{ft} \mathrm{W} \text { of } \\
\text { Col. } 27 \mathrm{~W}\end{array}$ \\
\hline VT968 & $27 \mathrm{~W}$ & $0.18 *$ & 0.18 & Other & $\begin{array}{l}\text { Top of steam line in coal room at } 5 \mathrm{ft} \mathrm{N} \& 2.5 \mathrm{ft} \mathrm{W} \\
\text { of Col. } 27 \mathrm{~W}\end{array}$ \\
\hline VT969 & $27 \mathrm{~W}$ & $0.32 *$ & 0.64 & Bridging & Bridging in coal room at $6 \mathrm{ft} \mathrm{N} \& 6 \mathrm{ft} \mathrm{W}$ of Col. $27 \mathrm{~W}$ \\
\hline VT970 & $27 \mathrm{~W}$ & $0.40 *$ & 0.40 & Wall & $\begin{array}{l}\text { Top of south block wall in the coal room at } 5 \mathrm{ft} \text { due } \\
W \text { of Col. } 27 \mathrm{~W}\end{array}$ \\
\hline VT971 & $27 \mathrm{~W}$ & $0.44 *$ & 0.44 & Wall & $\begin{array}{l}\text { Vertical surface (east wall in coal room) at } 5 \mathrm{ft} \text { due } \mathrm{N} \\
\text { of Col. } 27 \mathrm{~W}\end{array}$ \\
\hline VT973 & $27 \mathrm{~W}$ & $0.23^{*}$ & 0.23 & Wall & $\begin{array}{l}\text { Vertical surface (west wall in coal room) at } 5 \mathrm{ft} \text { and } 19 \\
\mathrm{ft} 1 \mathrm{in} \text {. W of Col.27W }\end{array}$ \\
\hline VT974 & $27 \mathrm{~W}$ & $0.28 *$ & 0.28 & Wall & $\begin{array}{l}\text { Vertical surface (south wall in coal room) at } 14 \mathrm{ft} \text { due } \\
W \text { of Col. } 27 \mathrm{~W}\end{array}$ \\
\hline VT975 & $27 \mathrm{~W}$ & $0.38 *$ & 0.38 & Floor & $\begin{array}{l}\text { Floor (coal room) at } 3 \mathrm{ft} \mathrm{N} \mathrm{\&} 14 \mathrm{ft} \text { W of Col. } 27 \mathrm{~W} \\
\text { and at boiler room level }\end{array}$ \\
\hline VT976 & $27 \mathrm{~W}$ & $0.40 *$ & 0.40 & Floor & $\begin{array}{l}\text { Floor (coal room) at } 5 \mathrm{ft} \mathrm{N} \& 5.5 \mathrm{ft} W \text { of Col. } 27 \mathrm{~W} \\
\text { and at boiler room level }\end{array}$ \\
\hline VT985 & Drain 2 & $0.13 *$ & 0.26 & Drain & $\begin{array}{l}\text { Drain (smeared to vertical depth of } 6 \text { in.) at } 28 \mathrm{ft} \mathrm{W} \& \\
2 \mathrm{ft} N \text { of Col. } 25 \mathrm{E}\end{array}$ \\
\hline VT986 & Drain 56 & $0.19 *$ & 0.38 & Drain & $\begin{array}{l}\text { Drain (smeared to } 12 \text { in. vertical depth) at } 32 \mathrm{ft} \mathrm{S} \mathrm{\&} 6 \\
\mathrm{ft} E \text { of Col. } 5 \mathrm{~N}\end{array}$ \\
\hline VT987 & $26 \mathrm{~W}$ & $<0.10^{*}$ & $<0.10$ & Wall & $\begin{array}{l}\text { Top of block wall above boiler room S door at } 15 \mathrm{ft} \mathrm{S} \\
\& 11 \mathrm{ft} \mathrm{W} \text { of Col. } 26 \mathrm{~W}\end{array}$ \\
\hline VT988 & $27 \mathrm{~W}$ & $0.19 *$ & 0.19 & Other & $\begin{array}{l}\text { Top of condensate tank (boiler room overhead) at } 3 \mathrm{ft} \\
S \& 6 \mathrm{ft} W \text { of Col. } 27 \mathrm{~W}\end{array}$ \\
\hline VT989 & $27 \mathrm{~W}$ & $0.17 *$ & 0.17 & Bridging & $\begin{array}{l}\text { Both bridging slats (boiler room overhead) at } 1 \mathrm{ft} \mathrm{S} \mathrm{\&} \\
6 \mathrm{ft} \mathrm{W} \text { of Col. } 27 \mathrm{~W}\end{array}$ \\
\hline VT990 & $27 \mathrm{~W}$ & $<0.10 *$ & $<0.10$ & Other & $\begin{array}{l}\text { Top of the support beam of condensate tank at } 6.33 \mathrm{ft} \\
\mathrm{S} \& 8.5 \mathrm{ft} \mathrm{W} \text { of Col. } 27 \mathrm{~W}\end{array}$ \\
\hline VT991 & $26 \mathrm{~W}$ & $0.15 *$ & 0.15 & Other & $\begin{array}{l}\text { Top of steam line in boiler room overhead at } 19 \mathrm{ft} \mathrm{S} \\
\& 2.5 \mathrm{ft} \mathrm{W} \text { of Col. } 26 \mathrm{~W}\end{array}$ \\
\hline
\end{tabular}


Table 4. (continued)

\begin{tabular}{|c|c|c|c|c|c|}
\hline $\begin{array}{l}\text { Sample } \\
\text { ID } e\end{array}$ & $\begin{array}{c}\text { Bay/room/ } \\
\text { structure/ } \\
\text { areab }\end{array}$ & $\begin{array}{c}\mathrm{Be}^{c} \\
(\mu \mathrm{g} / \mathrm{g}) \\
(\mu \mathrm{g})^{*}\end{array}$ & $\begin{array}{c}\text { Be/area } \\
\left(\mu \mathrm{g} / \mathrm{ft}^{2}\right)^{d}\end{array}$ & $\begin{array}{l}\text { Location } \\
\text { in bay }\end{array}$ & Location notese \\
\hline VT992 & $26 \mathrm{~W}$ & $0.15 *$ & 0.15 & Bridging & $\begin{array}{l}\text { Both bridging slats in boiler room overhead at } 12 \mathrm{ft} \mathrm{S} \\
\& 13 \mathrm{ft} \mathrm{W} \text { of Col. } 26 \mathrm{~W}\end{array}$ \\
\hline VT993 & $27 \mathrm{~W}$ & $0.21 *$ & 0.21 & Floor & $\begin{array}{l}\text { Floor in boiler room (north end near steps) at } 7 \mathrm{ft} \mathrm{S} \mathrm{\&} \\
8.5 \mathrm{ft} \text { W of Col. } 27 \mathrm{~W}\end{array}$ \\
\hline VT995 & $27 \mathrm{~W}$ & $0.11 *$ & 0.11 & Floor & $\begin{array}{l}\text { Boiler room floor (north end of west boiler pit) at } 12 \\
\mathrm{ft} \mathrm{S} \mathrm{\&} 11 \mathrm{ft} W \text { of Col.27W }\end{array}$ \\
\hline VT996 & $26 \mathrm{~W}$ & $0.21 *$ & 0.21 & Floor & Boiler $\mathrm{rm}$. (W boiler pit) at $12 \mathrm{ft}$ due W of Col. $26 \mathrm{~W}$ \\
\hline VT997 & $26 \mathrm{~W}$ & $0.13 *$ & 0.13 & Floor & Boiler $\mathrm{rm}$. (E boiler pipe) at $4 \mathrm{ft}$ due $\mathrm{W}$ of Col. $26 \mathrm{~W}$ \\
\hline VT998 & $26 \mathrm{~W}$ & $0.43 *$ & 0.43 & Floor & $\begin{array}{l}\text { Top of concrete separator on boiler room floor at } 9.33 \\
\mathrm{ft} \text { due W of Col. } 26 \mathrm{~W}\end{array}$ \\
\hline VT999 & $26 \mathrm{~W}$ & $0.17 *$ & 0.17 & Floor & $\begin{array}{l}\text { Boiler room floor (south end near S entrance) at } 12 \mathrm{ft} \\
\mathrm{S} \& 11 \mathrm{ft} \text { W of Col. } 26 \mathrm{~W}\end{array}$ \\
\hline VT1001 & $26 \mathrm{~W}$ & $0.12 *$ & 0.12 & $\begin{array}{l}\text { Window } \\
\text { ledge }\end{array}$ & $\begin{array}{l}\text { West window ledge in boiler room at } 18.5 \mathrm{ft} \text { due } \mathrm{W} \text { of } \\
\text { Col. } 26 \mathrm{~W}\end{array}$ \\
\hline VT1002 & $27 \mathrm{~W}$ & $0.13 *$ & 0.13 & Other & $\begin{array}{l}\text { East sidewall of north end sump pit (E boiler pit) at } 9 \\
\mathrm{ft} \& 5 \text { in. S of Col. } 27 \mathrm{~W}\end{array}$ \\
\hline VT1003 & Drain 37 & $<0.05 *$ & $<0.20$ & Drain & $\begin{array}{l}\text { Drain ( } 2 \text { in. vertical) at } 3.5 \mathrm{ft} \mathrm{N} \& 17 \mathrm{ft} \mathrm{E} \text { of Col. } \\
25 \mathrm{~W} \text {; a recheck of VT984 }\end{array}$ \\
\hline VT1004 & $30 \mathrm{~W}$ & $<0.05 *$ & $<0.05$ & Wall & $\begin{array}{l}\text { Fire header closet wall (vert. surface) at } 3.58 \mathrm{ft} \mathrm{S} \text { and } \\
19.25 \mathrm{ft} \mathrm{W} \text { of Col. } 30 \mathrm{~W}\end{array}$ \\
\hline VT1005 & $32 W$ & $<0.05 *$ & $<0.05$ & Wall & $\begin{array}{l}\text { West vertical surface of wall at } 1 \mathrm{ft} \& 2 \mathrm{in} . S \text { and } \\
19.25 \mathrm{ft} \text { W of Col. } 32 \mathrm{~W}\end{array}$ \\
\hline VT1006 & Drain 25 & $f$ & $<0.05$ & Drain & Sidewalls of drainpipe \\
\hline VT1007 & Drain 25 & $f$ & $<0.05$ & Drain & Sidewalls of drainpipe \\
\hline
\end{tabular}

"Samples designated "VM" are dust/debris or insulation; "VT" samples are smears. Locations are shown on Figs. 3 through 7. Not all numbers in the sample numbering system were used. Most gaps in the sample numbering system can be accounted for by the fact that much of the supplementary survey, remedial activities, and the verification survey were conducted simultaneously. This document and the document relating the details of the supplementary characterization survey [ORNL-6623 (Supplement)] considered together, contain all the data collected to assess the condition of the site.

${ }^{b}$ Beams are numbered; N, S, E. W., C designate north, south, east, west, and center; MS = metal shop; WS = wood shop.

'Analytical results showing concentrations of beryllium are in $\mu \mathrm{g} / \mathrm{g}$ unless otherwise indicated; i.e., ${ }^{*}=\mu \mathrm{g}$.

${ }^{d}$ Concentrations of beryllium in $\mu \mathrm{g} / \mathrm{ft}^{2}$ are the result of calculations performed using the sample size.

eAdditional commentary for entries preceded by an asterisk is given in Table 4.

No calculation/analysis performed. 
Table 5. Additional notations for selected indoor samples collected for the independent verification survey of $\mathbf{4 2 5}$ Peek Street

\begin{tabular}{|c|c|c|c|}
\hline $\begin{array}{l}\text { Sample } \\
\text { ID }{ }^{\circ}\end{array}$ & $\begin{array}{l}\text { Room/ } \\
\text { structure/ } \mathrm{Lc} \\
\text { area }^{b}\end{array}$ & & Location notese \\
\hline VM405 & Drain 10 & Other & Only upper 2 in. of pipe can be probed, msrments at step down. \\
\hline VM406 & Drain 10 & Other & $\begin{array}{l}\text { Composite sample in interval } 3 \text { to } 8 \text { in. (involving walls) and neck of } \\
\text { drain pipe at } 11 \text { in. }\end{array}$ \\
\hline VM407 & Drain 11 & Other & $\begin{array}{l}\text { No probe beyond } 2 \text { in. msments at step-down ( } 2 \text { in.) sample at } 3-8 \\
\text { in. interval. }\end{array}$ \\
\hline VM415 & Drain 17 & Other & Surface readings taken at top 2 in. of pipe. Sample depth $=0-1.5 \mathrm{in}$. \\
\hline VM417 & Drain 18 & Other & $\begin{array}{l}\text { Surface readings taken from bottom of pit }(2 \mathrm{ft} \times 2 \mathrm{ft}) \text {, sample depth } \\
=32 \text { in. }\end{array}$ \\
\hline VM419 & Drain 24 & Other & Surface readings taken from larger of 2 drain openings (B) 13 in. \\
\hline VM420 & Drain 24 & Other & $\begin{array}{l}\text { It appears that Pipe } A \text { is connected to Pipe } B \text { by a conducting pipe } \\
\text { sloping downward toward pipe } B \text {. }\end{array}$ \\
\hline VM421 & Drain 25 & Other & Surface readings taken at the top of 1.5 in. pipe. \\
\hline VM426 & Drain 49 & Other & Surface readings taken at top 2 in. of drain. \\
\hline VM428 & Drain 26 & Other & Surface readings taken at $\sim 50$ in., appears to be clay pipe at 12 in. \\
\hline VM443 & $31 \mathrm{~W}$ & Floor & $\begin{array}{l}\text { Floor dust of crane rail track near ( } 7 \mathrm{ft} \text { E \& } 3 \mathrm{ft} 4 \mathrm{in} . \mathrm{N} \text { ) Col. } 31 \mathrm{~W} \text {. } \\
\text { Rad. readings (beta-gamma) were taken at this sample site. Sample } \\
\text { was aliquoted and the duplicate (for ORNL) was assigned VM433A }\end{array}$ \\
\hline VM444 & $32 \mathrm{E}$ & Floor & $\begin{array}{l}\text { Floor dust under the gas bottle rack at } 6 \mathrm{in.} W \& 1 \mathrm{ft} 7 \mathrm{in} . \mathrm{N} \text { of } \mathrm{Col} \text {. } \\
\text { 32E. Rad. readings (beta-gamma \& alpha) were made at this site. } \\
\text { sample was split and the duplicate was assigned (ORNL) VM444A. }\end{array}$ \\
\hline VM445 & $38 \mathrm{~W}$ & Floor & $\begin{array}{l}\text { Floor depression near ( } 20 \mathrm{ft} 11 \text { in. E \& } 5 \mathrm{ft} 3 \text { in. N) Col. } 38 \mathrm{~W} \text {. Rad } \\
\text { readings (beta-gamma \& alpha) were taken and the sample was } \\
\text { aliquoted so ORNL could have a duplicate (VM445A) specimen. }\end{array}$ \\
\hline VM446 & $34 \mathrm{E}$ & Floor & $\begin{array}{l}\text { Floor dust from under the transformer near ( } 4 \mathrm{ft} 10 \mathrm{in} \text {. } \mathrm{N} \& 1 \mathrm{ft} 11 \\
\text { in. W) Col. } 34 \mathrm{E} \text {. This sample was aliquoted so ORNL could have the } \\
\text { duplicate (VM446A) specimen. Rad. readings were taken (beta- } \\
\text { gamma \& alpha) at the site. }\end{array}$ \\
\hline VM449 & $36 \mathrm{~W}$ & Floor & $\begin{array}{l}\text { This material is a floor coating of paint \& etc. scraped from the } \\
\text { original floor. }\end{array}$ \\
\hline VM450 & $39 W$ & Floor & $\begin{array}{l}\text { This material is a floor coating of paint \& etc. scraped from the } \\
\text { original floor. }\end{array}$ \\
\hline VM451 & $40 W$ & Floor & $\begin{array}{l}\text { This material is a floor coating of paint \& etc. scraped from the } \\
\text { original floor. }\end{array}$ \\
\hline VM452 & $39 W$ & Floor & $\begin{array}{l}\text { This material is a floor coating of paint \& etc. scraped from the } \\
\text { original floor. }\end{array}$ \\
\hline VM453 & $36 W$ & Floor & $\begin{array}{l}\text { This material is a floor coating of paint \& etc. scraped from the } \\
\text { original floor. }\end{array}$ \\
\hline
\end{tabular}


Table 5. (continued)

\begin{tabular}{|c|c|c|}
\hline $\begin{array}{c}\text { Sample } \\
\text { ID } a\end{array}$ & $\begin{array}{l}\text { Room/ } \\
\text { structure/ Location } \\
\text { area }^{b} \text { in bay }\end{array}$ & Location notesc \\
\hline
\end{tabular}

VM457 Drain 4 Drain The sample was taken at north access (5 ft 6 in. below floor) to Drain 3 which is located $26 \mathrm{ft} \mathrm{W} \& 4 \mathrm{ft} \mathrm{S}$ of $\mathrm{Col}$. 23E and was divided into 2 aliquots to provide ORNL with a rad sample (VM458). VM457 is for Be analysis.

VM461 Drain 2 Drain The sample was taken at the base of Drain 2 south access.

VM464 38W Floor This location is $9.5 \mathrm{ft} \mathrm{W}$ and $10.5 \mathrm{ft} \mathrm{S}$ of Col. 38W.

VM465 $38 \mathrm{~W} \quad$ Floor The sample location is the refrigeration room original floor at $9.5 \mathrm{ft}$ $\mathrm{W}$ and $10 \mathrm{ft} \mathrm{N}$ of $\mathrm{Col}$. $38 \mathrm{~W}$. A photograph was made of this sampling location.

VM466 39W Floor This refrigeration room original floor (after removal of top concrete \& styrofoam coatings) is located $9.5 \mathrm{ft} W$ and $10 \mathrm{ft} \mathrm{N}$ of Col. $39 \mathrm{~W}$.

VM472 23E Drain This location is $25 \mathrm{ft} \& 6$ in. W and $1 \mathrm{ft} \mathrm{S}$ of Col. 23E and is the

VM476 2S Drain VM473 was taken at this location for rad \& gamma spec. analysis

VM477 2S Drain

VM479 Drain $52 \quad$ Drain

VM483 2S Drain

VM487 27E Drain

Drain

VM489 2S Drain

VM490 24W Drain same site as sample VM471.

Sample VM474 was taken at this location for rad analysis.

N.D. on beta-gamma scan. Sample VT844 taken at this location for rad analysis.

N.D. on the beta-gamma scan; sample VM482 taken at this location for rad analysis.

Sample VM488 was taken at this location for rad analysis.

N.D. on the beta-gamma scan;Sample VM484 was taken at this same location for rad analysis.

Galvanized floor drain with an elbow at 16 in. (sample scooped from debris at this depth) and turning due E. Location is $4 \mathrm{ft} \mathrm{N} \& 8 \mathrm{ft} E$ of Col. 24W at extreme west wall of MS-1 and connects to location of VT896. Sample VT897 was taken at the 4 in. depth.

VM492 24W Drain

Composite collected at all depths up to $2 \mathrm{ft}$ from side walls of 4 in. diameter cast iron pipe with elbow at $24 \mathrm{in}$. which tums due east. Location is at the extreme west wall of MS-1 \& connects to location of VT897 \& VM490.

VM493 32W Drain

2-in. diameter galvanized drain pipe running 12 in. horizontal south before turming toward the floor (elbow) at the base of Col. $32 \mathrm{~W}$. Ref. KAPL 69 for rad scan to a depth of 12 in. Scoop-composite sample taken along entire $12 \mathrm{in.} \mathrm{length} \mathrm{of} \mathrm{bottom} \mathrm{radius} \mathrm{of} \mathrm{pipe.}$

VM494 32W Drain

3-in. diameter black iron pipe 16 in. deep to debris (trash) obstruction. Sample collected from entire side walls up to the obstruction. See VM495 for the sample for rad analysis at this location. 
Table 5. (continued)

\begin{tabular}{ccc}
\hline $\begin{array}{c}\text { Roomple } \\
\text { ID } a\end{array}$ & $\begin{array}{c}\text { structure/ } \\
\text { area }^{b}\end{array}$ in bay & Location notes \\
\hline
\end{tabular}

VM496 44E Drain

4-in. diameter cast iron Drain pipe (length undetermined) to main trunkline near Col. 44E (flat wall). Scoop sample taken on bottom radius side wall at horizontal length of 30 in. Ref. KAPL 63 connecting to KAPL 62. VM513 taken at this location for rad

VM497 42W Drain 2 in. diameter cast iron drain pipe at Col. $42 \mathrm{~W}$ in SE corner of MS-8. Sample collected from debris in horizontal access; vertical access obstructed by rust, debris, \& etc. VM498 is the rad sample collected at this location. Ref. KAPL 77.

VM499 37W Drain

2 in. diameter galvanized pipe roof Drain 2 near Col. 37W (extreme west column in refrig. room) had sample scraped from side wall to a $3.5 \mathrm{ft}$ depth with tape attached to snake lead. rad scan depth to 2 in. due to horizon. entrance access. Ref. KAPL 69

VM502 26W Drain

This 3 in. cast iron horizontal drain line opening is about $1.5 \mathrm{ft}$ below the floor and runs NE to SE. This sanitary line is clogged with debris about 6 in. inside. This is the same location as VM501 (a rad sample with N.D. above BG for the beta-gamma)

VM504 26W Drain

This is a 4 in. cast iron drain. The beta-gamma scan is N.D. above BG $(37 \mathrm{cpm} ;=0.02 \mathrm{mrad} / \mathrm{h})$ and sample VM503 was taken at this same location for rad analysis. The Be will be reported in ugms. per gm. only.

VM506 26W Drain

This drain line opening is about $2 \mathrm{ft}$ below the floor level and is clogged with debris. The beta-gamma scan was N.D. above BG ( 37 $\mathrm{cpm} ;=0.02 \mathrm{mrad} / \mathrm{h}$ ) and sample VM505 was taken at the same location for rad analysis. The Be will be reported as ugms. per gm. only.

VM507 27W Drain

This south-running 4-in. cast iron drain opening is about $4 \mathrm{ft}$ below floor level and was logged for radon 12-11-93. The beta-gamma scan was N.D. above BG $(37 \mathrm{cpm} ;=0.02 \mathrm{mrad} / \mathrm{h})$ and sample VT883 (lack of sample required a smear) was taken at this location for rad

VM508 30E Drain

This drain line runs west to east and is about $2 \mathrm{ft}$ below the floor level. VT884 was smeared for rad analysis at this location as no debris was available to sample.

VM510 34W Drain

The 4 in. diameter cast iron pipe runs north to south and is about $3 \mathrm{ft}$ below floor level. Sample VM509 was collected at this location for rad analysis.

VM514 4N Drain

This 1.5 -in. diameter pipe is 14 in. deep to elbow and turns south. The sample was taken from the side walls and elbow debris at a 14 in. depth and is a resample of VM480. Ref. KAPL 53. The betagamma scan was N.D. above BG $(40 \mathrm{cpm} ;=0.02 \mathrm{mrad} / \mathrm{h})$.

VM515 Stack Floor VT949 was taken for rad analysis near this location (south of VM515). 
Table 5. (continued)

\begin{tabular}{|c|c|c|c|}
\hline $\begin{array}{l}\text { Sample } \\
\text { ID } a\end{array}$ & $\begin{array}{l}\text { Room/ } \\
\text { structure/ } \\
\text { area }^{b}\end{array}$ & $\begin{array}{c}\text { Location } \\
\text { in bay }\end{array}$ & Location notesc \\
\hline VM517 & Stack & Wall & $\begin{array}{l}\text { This sample was taken directly east of VM516 (the sample for rad } \\
\text { analysis). }\end{array}$ \\
\hline VM518 & Stack & Wall & $\begin{array}{l}\text { This location had a beta-gamma background of } 120 \mathrm{cpm} \\
(0.06 \mathrm{mrad} / \mathrm{h} \text { ) taken in the center of stack with probe facing upward. } \\
\text { The rad sample taken near this location (north side of VM518 } \\
\text { abutting edge) is VT952. }\end{array}$ \\
\hline VM519 & Stack & Wall & $\begin{array}{l}\text { This location had a beta-gamma background of } 98 \mathrm{cpm} \\
(0.05 \mathrm{mrad} / \mathrm{h} \text { ) with the probe facing up. Sample VT954 was taken } \\
\text { near this location (directly east) for rad analysis. }\end{array}$ \\
\hline VM520 & Stack & Wall & $\begin{array}{l}\text { The beta-gamma background was } 72 \mathrm{cpm}(0.04 \mathrm{mrad} / \mathrm{h}) \text { with the } \\
\text { probe facing up. Sample VM521 was taken directly above VM520 } \\
\text { for rad analysis. }\end{array}$ \\
\hline VM522 & Stack & Wall & $\begin{array}{l}\text { The beta-gamma background with the probe facing up was } 64 \mathrm{cpm} \\
(0.03 \mathrm{mrad} / \mathrm{h}) \text { at this location. }\end{array}$ \\
\hline VM523 & Stack & Wall & $\begin{array}{l}\text { Brick was sawed to collect the sample which will serve as a } \\
\text { background sample as it was never exposed to the environs. Beta- } \\
\text { gamma scan was } 59 \mathrm{cpm}(10 \mathrm{cpm} \text { above } B G=0.03 \mathrm{mrad} / \mathrm{h}) \text { at this } \\
\text { location and sample VM524 is the rad sample for this location. }\end{array}$ \\
\hline
\end{tabular}

VM525 Drain 51 Drain

This 3-in. iron pipe (possibly located near Col. 3N) had kerosene present and appears to have been KAPL cleaned before smear and sample collection. Sample VT977 was taken in the drain basin (15.5 ft $\mathrm{N} \& 10 \mathrm{ft} E$ of Col. $3 \mathrm{~N}$ ) for rad analysis.

VM526 Stack Wall

This sample was red brick from the wall to be used to determine background Be content of the material. The location was $2 \mathrm{ft} N$ of SE corner of room at $5 \mathrm{ft}$ above the concrete floor. VM527 was collected for rad background determination at this location

VM528 Boiler Boiler Beta-gamma scan N.D. above background. Sample VM530 was taken nearby for rad analysis.

VM529 Boiler Boiler Beta-gamma scan N.D. above background. Sample VM531 was taken nearby for rad analysis.

VM532 26W Drain

This 4 in. diameter drain (depth not taken) is located in the south end of the boiler room. The beta-gamma scan is N.D. above background.

VM533 27W Drain

This 4 inch diameter drain (depth not taken) is located in the boiler room at $4 \mathrm{ft} \mathrm{S} \mathrm{\&} 1 \mathrm{ft} \mathrm{W}$ of Col. $27 \mathrm{~W}$. The beta-gamma scan was N.D. above background.

VT270 Drain 09 Other

"Smears 2 in. - 6 in. depth; insufficient mat'l for sample" This was intended for Be analysis.

VT272 Drain 12 Other "Insuff. mat'l for sample; debris at bottom could not be gotten; smeared at 2 in. stepdown"

VT274 Drain $14 \quad$ Other

"smeared where probed; bottom obscured by debris (regular trash: cups,etc.)" 
Table 5. (continued)

\begin{tabular}{|c|c|c|c|}
\hline $\begin{array}{l}\text { Sample } \\
\text { ID }\end{array}$ & $\begin{array}{l}\text { Room/ } \\
\text { structure/ Loc } \\
\text { area }^{b} \text { in }\end{array}$ & & Location notesc \\
\hline VT279 & NE comer & Floor & Location was scanned for beta-gamma and alpha before smearing. \\
\hline VT280 & $43 E$ & Floor & Location scanned for beta-gamma \& alpha before smearing. \\
\hline VT281 & $42 \mathrm{E}$ & Floor & Location was scanned for beta-gamma \& alpha before smearing. \\
\hline VT282 & $41 \mathrm{E}$ & Floor & Location was scanned for beta-gamma \& alpha prior to smearing. \\
\hline VT283 & $40 E$ & Floor & Location was scanned for beta-gamma \& alpha before smearing. \\
\hline VT284 & $39 \mathrm{E}$ & Floor & Location was scanned for beta-gamma \& alpha prior to smearing. \\
\hline VT285 & $38 \mathrm{E}$ & Floor & Location was scanned for beta-gamma \& alpha before smearing. \\
\hline VT286 & $37 E$ & Floor & Location was scanned for beta-gamma \& alpha before smearing. \\
\hline VT287 & $36 \mathrm{E}$ & Floor & Location was scanned for beta-gamma \& alpha before smearing. \\
\hline VT288 & $35 \mathrm{E}$ & Floor & Location was scanned for beta-gamma \& alpha before smearing. \\
\hline VT289 & $34 \mathrm{E}$ & Floor & Location was scanned for beta-gamma \& alpha before smearing. \\
\hline VT290 & $33 \mathrm{E}$ & Floor & Location scanned for beta-gamma \& alpha before smearing. \\
\hline VT291 & $32 \mathrm{E}$ & Floor & Location was scanned for beta-gamma \& alpha before smearing. \\
\hline VT292 & $32 \mathrm{~W}$ & Floor & Location was scanned for beta-gamma \& alpha before smearing. \\
\hline VT293 & $33 \mathrm{~W}$ & Floor & Location was scanned for beta-gamma \& alpha before smearing. \\
\hline VT294 & $34 \mathrm{~W}$ & Floor & Location was scanned for beta-gamma \& alpha before smearing. \\
\hline VT295 & $35 \mathrm{~W}$ & Floor & Location was scanned for beta-gamma \& alpha before smearing. \\
\hline VT296 & $36 \mathrm{~W}$ & Floor & Location was scanned for beta-gamma \& alpha before smearing. \\
\hline VT297 & $37 \mathrm{~W}$ & Floor & Location was scanned for beta-gamma \& alpha before smearing. \\
\hline VT298 & $38 \mathrm{~W}$ & Floor & ocation was scanned for beta-gamma \& alpha before smearing. \\
\hline VT299 & $39 \mathrm{~W}$ & Floor & Location was scanned for beta-gamma \&alpha before smearing. \\
\hline VT300 & 40W & Floor & Location was scanned for beta-gamma \& alpha before smearing. \\
\hline T301 & $41 \mathrm{~W}$ & Floor & Location was scanned for beta-gamma \& alpha before smearing. \\
\hline VT302 & $42 \mathrm{~W}$ & Floor & Location was scanned for beta-gamma \&alpha before smearing. \\
\hline VT303 & $43 \mathrm{~W}$ & Floor & Location was scanned for beta-gamma \& alpha prior to smearing. \\
\hline VT304 & NW corner & Floor & $\begin{array}{l}\text { NW corner of bldg. Location was scanned for beta-gamma \& alpha } \\
\text { before smearing. }\end{array}$ \\
\hline VT305 & $43 \mathrm{~W}$ & Floor & Pre-smear beta-gamma \& alpha scans conducted. \\
\hline VT306 & $42 \mathrm{~W}$ & Floor & Pre-smear beta-gamma \& alpha scans conducted. \\
\hline VT307 & $41 \mathrm{~W}$ & Floor & Pre-smear beta-gamma \& alpha scans conducted. \\
\hline VT308 & $40 \mathrm{~W}$ & Floor & Pre-smear beta-gamma \& alpha scans conducted. \\
\hline VT309 & $40 \mathrm{~W}$ & Floor & Pre-smear beta-gamma \& alpha scans conducted. \\
\hline
\end{tabular}


Table 5. (continued)

\begin{tabular}{ccc}
\hline Room/ & \\
$\begin{array}{c}\text { Sample } \\
\text { ID } a\end{array}$ & $\begin{array}{c}\text { structure/ Location } \\
\operatorname{area}^{b}\end{array}$ in bay & Location notes \\
\hline
\end{tabular}

\begin{tabular}{|c|c|c|c|}
\hline VT310 & $39 \mathrm{~W}$ & Floor & Pre-smear beta-gamma \& alpha scans conducted. \\
\hline VT311 & $38 \mathrm{~W}$ & Floor & Pre-smear beta-gamma \& alpha scans conducted. \\
\hline VT312 & $37 \mathrm{~W}$ & Floor & Pre-smear beta-gamma \& alpha scans conducted. \\
\hline VT313 & $36 \mathrm{~W}$ & Floor & Pre-smear beta-gamma \& alpha scans conducted. \\
\hline VT314 & $31-32 E$ & Window & $\begin{array}{l}\text { Entire ledge ( } 2 \text { in. } \times 48 \text { in.) smeared of the middle low window } \\
\text { located between Col. } 31 \mathrm{E} \& 32 \mathrm{E} \text {. A pre-smear beta-gamma scan was } \\
\text { conducted. }\end{array}$ \\
\hline VT315 & 33-34E & Window & $\begin{array}{l}\text { Window ledge of the south low window located between column } \\
33 \mathrm{E} \& 34 \mathrm{E} \text {. A beta-gamma \& alpha scan was conducted before } \\
\text { smearing. }\end{array}$ \\
\hline VT316 & $35-36 \mathrm{E}$ & Window & $\begin{array}{l}\text { Entire ledge ( } 2 \text { in. } \times 48 \text { in.) smeared of the middle low window } \\
\text { located between Columns } 35 \mathrm{E} \& 36 \mathrm{E} \text {. A pre-smear beta-gamma } \\
\text { scan was conducted. }\end{array}$ \\
\hline VT317 & 37-38E & Window & $\begin{array}{l}\text { Entire ledge ( } 2 \text { in. } \times 48 \text { in.) smeared of the middle low window } \\
\text { located between Col. } 37 \mathrm{E} \& 38 \mathrm{E} \text {. A pre-smear beta-gamma scan was } \\
\text { conducted. }\end{array}$ \\
\hline
\end{tabular}

VT318 $39-40 E$ Window Entire ledge (2in. $\times 48$ in.) smeared of the middle low window located between Col. 39E \& 40E. A pre-smear beta-gamma scan was conducted.

VT319 41-42E Window Entire ledge ( 2 in. $\times 48$ in.) smeared of the middle low window located between Col. $41 \mathrm{E} \& 42 \mathrm{E}$. A pre-smear beta-gamma scan was conducted.

VT320 31W-32W Window Center window ledge located between Bay $31 \mathrm{~W} \& 32 \mathrm{~W}$. A presmear beta-gamma \& alpha scan was conducted.

VT321 31W-32W Wall Top of the concrete block wall between Bay $31 \mathrm{~W} \& 32 \mathrm{~W}$. A presmear beta-gamma \& alpha scan was conducted.

VT322 31W-32W Bridging Top of bridging (between stringers) and about one halfway toward top of peak between Bay $31 \mathrm{~W} \& 32 \mathrm{~W}$. A pre-smear beta-gamma \& alpha scan was conducted.

VT323 31W Truss Top of truss (about one half toward peak) located east of Column 31 W. A pre mear beta-gamma \& alpha scan was conducted.

VT324 32W-33W Window Center window ledge located between Bay 32W \& 33W. A presmear beta-gamma \& alpha scan was conducted.

VT325 32W-33W Wall Top of concrete block wall between Bay 32W \& 33W. A pre-smear beta-gamma \& alpha scan was conducted.

VT326 32W-33W Bridging Top of bridging (between stringers) about one half way up and located between Bay $32 \mathrm{~W} \& 33 \mathrm{~W}$. A pre-smear beta-gamma \& alpha scan was conducted.

VT327 $32 \mathrm{~W}$ Truss A pre-smear beta-gamma \& alpha scan was conducted. 
Table 5. (continued)

\begin{tabular}{|c|c|c|c|}
\hline $\begin{array}{l}\text { Sample } \\
\text { IDa }\end{array}$ & $\begin{array}{l}\text { Room/ } \\
\text { structure/ Loc } \\
\text { areab in }\end{array}$ & & Location notesc \\
\hline VT328 & $33 W-34 W$ & Window & $\begin{array}{l}\text { Top of center window ledge located between Bay } 33 \mathrm{~W} \& 34 \mathrm{~W} \text {. A } \\
\text { pre-smear beta-gamma \& alpha scan was conducted. }\end{array}$ \\
\hline VT329 & $33 W-34 W$ & Stringer & $\begin{array}{l}\text { Side of stringer (in opening between and above top of block wall and } \\
\text { roof decking) located between Bay } 33 \mathrm{~W} \& 34 \mathrm{~W} \text {. This stringer is } \\
\text { visibly dirty. A pre-smear beta-gamma scan was conducted. }\end{array}$ \\
\hline VT330 & $43 \mathrm{E}$ & Window & Pre-smear beta-gamma \& alpha scans conducted. \\
\hline VT331 & $31 E$ & Floor & Pre-smear beta-gamma \& alpha scan conducted. \\
\hline VT332 & $30 E$ & Floor & Pre-smear beta-gamma \& alpha scan conducted. \\
\hline VT333 & $29 \mathrm{E}$ & Floor & Pre-smear beta-gamma \& alpha scan conducted. \\
\hline VT334 & $28 \mathrm{E}$ & Floor & Pre-smear beta-gamma \& alpha scan conducted. \\
\hline VT335 & $27 \mathrm{E}$ & Floor & Pre-smear beta-gamma \& alpha scans conducted. \\
\hline VT336 & $26 \mathrm{E}$ & Floor & Pre-smear beta-gamma \& alpha scans conducted. \\
\hline VT337 & $25 \mathrm{E}$ & Floor & Pre-smear beta-gamma \& alpha scans conducted. \\
\hline VT338 & 24E & Floor & Pre-smear beta-gamma \& alpha scans conducted. \\
\hline VT339 & $23 \mathrm{E}$ & Floor & Pre-smear beta-gamma \& alpha scans conducted. \\
\hline VT340 & $22 \mathrm{E}$ & Floor & Pre-smear beta-gamma \& alpha scans conducted. \\
\hline VT341 & $22 W$ & Floor & Pre-smear beta-gamma \& alpha scans conducted. \\
\hline VT342 & $22 W$ & Floor & Pre-smear beta-gamma \& alpha scans conducted. \\
\hline VT343 & $23 \mathrm{~W}$ & Floor & Pre-smear beta-gamma \& alpha scans conducted. \\
\hline VT344 & $23 W$ & Floor & Pre-smear beta-gamma \& alpha scans conducted. \\
\hline VT345 & $24 W$ & Floor & Pre-smear beta-gamma \& alpha scans conducted. \\
\hline VT346 & $25 \mathrm{~W}$ & Floor & Pre-smear beta-gamma \& alpha scans conducted. \\
\hline VT347 & $26 \mathrm{~W}$ & Floor & Pre-smear beta-gamma \& alpha scans conducted. \\
\hline VT348 & $27 \mathrm{~W}$ & Floor & Pre-smear beta-gamma \& alpha scans conducted. \\
\hline VT349 & $28 \mathrm{~W}$ & Floor & Pre-smear beta-gamma $\&$ alpha scans conducted. \\
\hline VT350 & $29 W$ & Floor & Pre-smear beta-gamma \& alpha scans conducted. \\
\hline VT351 & $30 \mathrm{~W}$ & Floor & Pre-smear beta-gamma \& alpha scans conducted. \\
\hline VT352 & $31 \mathrm{~W}$ & Floor & Pre-smear beta-gamma \& alpha scans conducted. \\
\hline VT353 & $29-30 \mathrm{E}$ & Window & $\begin{array}{l}\text { Entire ledge ( } 2 \text { in. } \times 48 \text { in.) smeared of the low middle window } \\
\text { located between Cols. } 29 \mathrm{E} \& 30 \mathrm{E} \text {. Pre-smear beta-gamma scans } \\
\text { were conducted. }\end{array}$ \\
\hline VT354 & $27-28 \mathrm{E}$ & Window & $\begin{array}{l}\text { Entire ledge ( } 2 \text { in } \times 48 \text { in.) smeared of the low middle window } \\
\text { located between Col. } 27 \mathrm{E} \& 28 \mathrm{E} \text {. Pre-smear beta-gamma } \& \text { alpha } \\
\text { scans were conducted. }\end{array}$ \\
\hline
\end{tabular}


Table 5. (continued)

\begin{tabular}{ccc}
\hline Room/ & & \\
$\begin{array}{c}\text { Sample } \\
\text { ID } a\end{array}$ & $\begin{array}{c}\text { structure/ Location } \\
\text { area }^{b}\end{array}$ in bay & Location notes $^{c}$ \\
\hline
\end{tabular}

$\begin{array}{llll}\text { VT355 } & \text { 25-26E } & \text { Window } & \begin{array}{l}\text { Entire ledge }(2 \text { in. } \times 48 \text { in.) smeared of the low north window located } \\ \text { between Col. } 25 \mathrm{E} \& 26 \mathrm{E} \text {. Pre-smear beta-gamma \& alpha scans } \\ \text { conducted. }\end{array} \\ \text { VT356 } & \text { 25-24E } & \text { Window } & \begin{array}{l}\text { Low window ledge between Col. 25E \& 24E. Pre-smear beta-gamma } \\ \& \text { alpha scans conducted. }\end{array} \\ \text { VT357 } & \text { MS-1 } & \text { Floor } & \begin{array}{l}\text { Floor smear taken in room MS-1 (furnace room) at } 9 \mathrm{ft} \text { W and } 9 \mathrm{fft} \\ \text { Sof Col. 25W. Pre-smear beta-gamma \& alpha scans were } \\ \text { conducted. }\end{array} \\ \text { VT358 } & \text { MS-1 } & \text { Floor } & \begin{array}{l}\text { Floor smear taken } 7 \mathrm{ft} S \& 8 \mathrm{ft} \text { E of Col. 24W on west wall of Rm. } \\ \text { MS-1 (furnace room). Pre-smear beta-gamma \& alpha scans were } \\ \text { conducted. }\end{array}\end{array}$

VT359 28-29W Floor Floor smear taken $5 \mathrm{ft} \mathrm{W} \& 2 \mathrm{ft} \mathrm{N}$ of the $\mathrm{N}$ edge of the door frame to the men's room which is located west \& between Cols. $28 \mathrm{~W} \& 29 \mathrm{~W}$. Pre-smear beta-gamma \& alpha scans were conducted.
VT360 33W-34W Bridging Top of bridging (about one half way up to the peak) located between Bay 33W \& 34W.
VT361 33W Truss Top of truss (about one half way to peak) located east of Col. 33W.
VT362 34W Truss Top of lower horizontal member of truss about one half to center from Col. 34W.

VT363 34W-35W Other Top of lower cross brace going between trusses $34 \mathrm{~W} \& 35 \mathrm{~W}$ located about $2 \backslash 3$ way from $W$. wall.

VT364 $35 \mathrm{~W} \quad$ Truss

Top of truss (about 12 way to peak) east of Col. $35 \mathrm{~W}$. This is the next section above the old VM340

VT365 35W-36w Bridging

Top of bridging (about 12 way to peak) east from the wall between Bay $35 \mathrm{~W} \& 36 \mathrm{~W}$.

VT367 36W-37W Bridging Top of bridging (about 12 way to peak) between Bay $36 \mathrm{~W} \& 37 \mathrm{~W}$.

VT368 36W-37W Stringer Upper flat side of stringer (between trusses and about 12 way to peak) between $36 \mathrm{~W} \& 37 \mathrm{~W}$.

VT369 37W Truss This location is above the old VT068

VT370 37W-38W Stringer Top flat side of a stringer which is visibly dirty and about 12 way to the peak between Col. 37W \& $38 \mathrm{~W}$.

VT371 MS-6 Floor Pre-smear beta-gamma \& alpha scans were conducted.

VT372 MS-6 Window Smear from east side of window ledge in Rm. MS-6 which is located between Col. 25E \& 26E. Pre-smear beta-gamma scan was conducted.

VT373 MS-6 Floor Smear taken inside the room immed. N of the vault and $9 \mathrm{ft} S \& 8 \mathrm{ft}$ $\mathrm{E}$ of Col. 25E. Pre-smear beta-gamma \& alpha scans conducted. 
Table 5. (continued)

\begin{tabular}{|c|c|c|c|}
\hline $\begin{array}{c}\text { Sample } \\
\mathrm{ID} a\end{array}$ & $\begin{array}{l}\text { Room/ } \\
\text { structure/ Loc } \\
\text { area }^{b} \quad \text { in }\end{array}$ & $\begin{array}{l}\text { tion } \\
\text { bay }\end{array}$ & Location notes $c$ \\
\hline VT374 & VAULT & Floor & Pre-smear beta-gamma \& alpha scans conducted. \\
\hline VT375 & VAULT & Other & $\begin{array}{l}\text { Bottom upper-facing surface (west end, north lip) of the middle "I" } \\
\text { Beam in vault. Pre-smear beta-gamma scan conducted. }\end{array}$ \\
\hline VT376 & MS-6 & Other & $\begin{array}{l}\text { Bottom upper-facing surface (east end, south lip) of the north "I" } \\
\text { Beam in Rm. MS-6. Pre-smear beta-gamma scan conducted. }\end{array}$ \\
\hline VT377 & $31-32 \mathrm{E}$ & Wall & $\begin{array}{l}\text { Top of plate located between Col. 31E \& 32E. Pre-smear beta- } \\
\text { gamma scan conducted. }\end{array}$ \\
\hline VT378 & $32-33 E$ & Window & $\begin{array}{l}\text { Ledge of the upper middle window located between Col. 32E \& 33E. } \\
\text { Pre-smear beta-gamma scan conducted. }\end{array}$ \\
\hline VT379 & $32-33 \mathrm{E}$ & Wall & Pre-smear beta-gamma \& alpha scans conducted. \\
\hline VT380 & $33-34 \mathrm{E}$ & Wall & Pre-smear beta-gamma scan conducted. \\
\hline VT381 & $38 \mathrm{~W}$ & Truss & Top of truss about 12 way to the roof peak E of Column line $38 \mathrm{~W}$. \\
\hline VT382 & $38-39 W$ & Bridging & $\begin{array}{l}\text { Top of bridging about } 12 \text { to roof peak and east of and between Col. } \\
38 \mathrm{~W} \& 39 \mathrm{~W} \text {. }\end{array}$ \\
\hline VT383 & $40-41 \mathrm{~W}$ & Bridging & Top of bridging about 12 way to peak E and between $40 \mathrm{~W} \& 41 \mathrm{~W}$. \\
\hline VT384 & $41 \mathrm{~W}$ & Truss & Top of truss about 12 way to roof peak east of Col. line $41 \mathrm{~W}$. \\
\hline VT385 & $41 W-42 W$ & Bridging & Top of bridging about 12 way to peak; $E$ and between $41 \mathrm{~W} \& 42 \mathrm{~W}$. \\
\hline VT386 & $41 W-42 W$ & Wall & $\begin{array}{l}\text { Top of block wall between Col. } 41 \mathrm{~W} \& 42 \mathrm{~W} \text {. Sample taken after } \\
\text { additional remediation. }\end{array}$ \\
\hline VT387 & $41 W-42 W$ & Window & Center window ledge between Col. $41 \mathrm{~W} \& 42 \mathrm{~W}$. \\
\hline VT388 & $42 \mathrm{~W}$ & Truss & Top of truss about 12 way to roof peak; east of Col. $42 \mathrm{~W}$. \\
\hline VT389 & $42 W-43 W$ & Bridging & Top of bridging $~ 12$ way to roof peak; $E$ \& between $42 W \& 43 W$. \\
\hline VT390 & $42 W-43 W$ & Wall & Top of block wall between Col. $42 \mathrm{~W} \& 43 \mathrm{~W}$. \\
\hline VT391 & $42 W-43 W$ & Window & Center window ledge between Columns $42 \mathrm{~W} \& 43 \mathrm{~W}$. \\
\hline VT392 & $43 \mathrm{~W}$ & Window & South window ledge between Col. $43 \mathrm{~W}$ and $\mathrm{NW}$ corner of building. \\
\hline VT393 & 44E-44W & Wall & $\begin{array}{l}\text { Top of block wall on far north end of building about } 2 \mathrm{ft} \text { east of the } \\
\text { old VM } 373 \text {. }\end{array}$ \\
\hline VT395 & $29 \mathrm{~W}$ & Other & $\begin{array}{l}\text { Location was above a false ceiling with opening access located NW } \\
\text { of Col. } 29 \mathrm{~W} \text {. The surface of the steam line was visibly clean. }\end{array}$ \\
\hline VT396 & $29 \mathrm{~W}$ & Other & $\begin{array}{l}\text { The location was above a false ceiling; smear taken on the visibly } \\
\text { dirty upper horizontal surface of fire sprinkler line located about } 17 \\
\mathrm{ft} \mathrm{N} \text { and about } 6 \mathrm{ft} \mathrm{W} \text { of Col. } 29 \mathrm{~W} \text {. }\end{array}$ \\
\hline VT397 & $29 \mathrm{~W}$ & Other & $\begin{array}{l}\text { Above false ceiling and above opening in sheet rock at the old } \\
\text { location of VM124; smear taken on a visibly dirty wooden cross } \\
\text { brace between ceiling joists located about } 60 \mathrm{ft} \mathrm{N} \text { and } 3 \mathrm{ft} \mathrm{W} \text { of Col. } \\
29 \mathrm{~W} \text {. }\end{array}$ \\
\hline
\end{tabular}


Table 5. (continued)

\begin{tabular}{|c|c|c|c|}
\hline $\begin{array}{l}\text { Sample } \\
\text { ID } a\end{array}$ & $\begin{array}{c}\text { Room/ } \\
\text { structure/ LC } \\
\text { areab }\end{array}$ & $\begin{array}{l}\text { Location } \\
\text { in bay }\end{array}$ & Location notesc \\
\hline VT398 & $29 \mathrm{~W}$ & Bridging & $\begin{array}{l}\text { Above false ceiling; smear taken on visibly dirty bridging between } \\
\text { ceiling joists located about } 80 \mathrm{ft} \mathrm{N} \text { and about } 7 \mathrm{ftW} \text { of Col. } 29 \mathrm{~W} \text {. }\end{array}$ \\
\hline VT399 & $34-35 W$ & Window & Window ledge (center) between Col. $34 \mathrm{~W} \& 35 \mathrm{~W}$ on wood frame. \\
\hline VT400 & $34-35 W$ & Stringer & $\begin{array}{l}\text { Vertical board (? stringer) west of the top wood plate at top of block } \\
\text { wall between Col. } 34 \mathrm{~W} \& 35 \mathrm{~W} \text {. }\end{array}$ \\
\hline VT401 & 34-35E & Wall & Pre-smear beta-gamma \& alpha scans conducted. \\
\hline VT402 & $34-35 E$ & $\begin{array}{l}\text { Window } \\
\text { ledge }\end{array}$ & $\begin{array}{l}\text { Ledge of the upper middle window located between Col. } 34 \mathrm{E} \& 35 \mathrm{E} \text {. } \\
\text { Pre-smear beta-gamma scan conducted. }\end{array}$ \\
\hline VT403 & 35-36E & Wall & Pre-smear beta-gamma \& alpha scans conducted. \\
\hline VT404 & 36-37E & Wall & Pre-smear beta-gamma \& alpha scans conducted. \\
\hline VT405 & $36-37 E$ & $\begin{array}{l}\text { Window } \\
\text { ledge }\end{array}$ & $\begin{array}{l}\text { Upper window ledge between Col. } 36 \mathrm{E} \& 37 \mathrm{E} \text {. Pre-smear beta- } \\
\text { gamma scan conducted. }\end{array}$ \\
\hline VT406 & $37-38 \mathrm{E}$ & Wall & Pre-smear beta-gamma \& alpha scans conducted. \\
\hline VT407 & $38-39 E$ & Wall & Pre-smear beta-gamma scan conducted. \\
\hline VT408 & $38-39 E$ & Window & $\begin{array}{l}\text { Upper south window between Col. 38E \& 39E. Pre-smear beta- } \\
\text { gamma scan conducted. }\end{array}$ \\
\hline VT409 & $39-40 \mathrm{E}$ & Wall & Pre-smear beta-gamma \& alpha scans conducted. \\
\hline VT410 & $40-41 \mathrm{E}$ & Wall & Pre-smear beta-gamma \& alpha scans conducted. \\
\hline VT411 & $40-41 E$ & Window & $\begin{array}{l}\text { Upper middle window between Cols. } 40 E \& 41 \mathrm{E} \text {. Pre-smear beta- } \\
\text { gamma scans conducted. }\end{array}$ \\
\hline VT412 & $41-42 E$ & Wall & Pre-smear beta-gamma \& alpha scans conducted. \\
\hline VT413 & $42-43 E$ & Wall & Pre-smear beta-gamma \& alpha scans conducted. \\
\hline VT414 & $42-43 \mathrm{E}$ & Window & $\begin{array}{l}\text { Upper middle window between Col. } 42 \mathrm{E} \& 43 \mathrm{E} \text {. Pre-smear beta- } \\
\text { gamma scan conducted. }\end{array}$ \\
\hline VT415 & $43 \mathrm{E}$ & Wall & $\begin{array}{l}\text { Top of plate between Col. } 43 \mathrm{E} \text { and the end of the building. Pre- } \\
\text { smear beta-gamma scan conducted. }\end{array}$ \\
\hline VT416 & $33-34 \mathrm{~W}$ & Stringer & $\begin{array}{l}\text { Vertical board (? stringer) above the wood plate at top of block wall } \\
\text { between Col. } 33 \mathrm{~W} \& 34 \mathrm{~W} \text { located one ft directly south of VT329. }\end{array}$ \\
\hline VT417 & $35 \mathrm{~W}$ & Other & $\begin{array}{l}\text { Top of heater (suspended from ceiling) located about 13' east of Col. } \\
35 \mathrm{~W} \text {. }\end{array}$ \\
\hline VT418 & $35-36 \mathrm{~W}$ & Window & $\begin{array}{l}\text { Top of center window ledge between Col. } 35 \mathrm{~W} \& 36 \mathrm{~W} \text { on wood } \\
\text { frame. }\end{array}$ \\
\hline VT420 & $36-37 \mathrm{~W}$ & Window & $\begin{array}{l}\text { Top of center window ledge between Col. } 36 \mathrm{~W} \& 37 \mathrm{~W} \text { on wood } \\
\text { frame. }\end{array}$ \\
\hline VT421 & $36-37 \mathrm{~W}$ & Stringer & $\begin{array}{l}\text { Vertical board (? stringer) above wood plate at top of block wall } \\
\text { between Col. } 36 \mathrm{~W} \& 37 \mathrm{~W} \text {. }\end{array}$ \\
\hline
\end{tabular}


Table 5. (continued)

\begin{tabular}{|c|c|c|c|}
\hline $\begin{array}{l}\text { Sample } \\
\text { IDa }\end{array}$ & $\begin{array}{l}\text { Room/ } \\
\text { structure/ L } \\
\text { areab }^{b}\end{array}$ & $\begin{array}{l}\text { Location } \\
\text { in bay }\end{array}$ & Location notesc \\
\hline VT422 & $37-38 \mathrm{~W}$ & Window & Center window concrete ledge between Col. 37W \& 38W. \\
\hline VT423 & 43W & Truss & Top of truss about 113 way to the roof peak from Col. $43 \mathrm{~W}$. \\
\hline VT424 & $43 \mathrm{~W}$ & Wall & $\begin{array}{l}\text { Truss plate (wood) at top of block wall between Col. } 43 \mathrm{~W} \& \mathrm{NW} \\
\text { corner of building. }\end{array}$ \\
\hline VT425 & $43 \mathrm{~W}$ & Bridging & $\begin{array}{l}\text { Bridging about } 12 \text { way to the peak from wall between Col. } 43 \text { W \& } \\
\text { NW comer of building. }\end{array}$ \\
\hline VT426 & $40-41 \mathrm{~W}$ & Window & Center window ledge (wood) between Col $40 \mathrm{~W} \& 41 \mathrm{~W}$. \\
\hline VT429 & $37 \mathrm{~W}$ & Truss & $\begin{array}{l}\text { Top of truss about } 13 \text { way up to peak from wall between Col. } 37 \mathrm{~W} \\
\& 38 \mathrm{~W} \text { was the entry on the field data sheet. Edited on } 1-7-94 \text { for } \\
\text { top of truss from Col. } 37 \mathrm{~W} \text {. Appears to be near or at the location of } \\
\text { VT369. }\end{array}$ \\
\hline VT430 & $37-38 \mathrm{~W}$ & Stringer & $\begin{array}{l}\text { Upper side of stringer about } 1 / 2 \text { way to peak from wall and located } \\
\text { between Col. } 37 \mathrm{~W} \& 38 \mathrm{~W} \text {. This location is about } 2 \mathrm{ft} \mathrm{N} \text { of VT } 370 \text {. } \\
\text { Additional remediation was performed on } 10-13-93 \text {. }\end{array}$ \\
\hline VT431 & $37-38 \mathrm{~W}$ & Bridging & Bridging 112 way to peak from wall between Col. $37 \mathrm{~W} \& 38 \mathrm{~W}$ \\
\hline VT432 & $38-39 \mathrm{~W}$ & Window & Center window ledge (wood) between Col. $38 \mathrm{~W} \& 39 \mathrm{~W}$. \\
\hline VT433 & $38-39 \mathrm{~W}$ & Wall & $\begin{array}{l}\text { Top of block wall between Col. } 38 \mathrm{~W} \& 39 \mathrm{~W} \text {. This location is about } \\
1 \mathrm{ft} \mathrm{N} \text { of old VM } 326 \text {. }\end{array}$ \\
\hline VT434 & $31-32 E$ & Bridging & Bridging about 12 to peak between Col. $31 \mathrm{E} \& 32 \mathrm{E}$. \\
\hline VT435 & $31 \mathrm{E}$ & Truss & $\begin{array}{l}\text { Top of lower cross brace of truss about } 12 \text { way from wall to peak } \\
\text { and west of Col. } 31 \mathrm{E} \text {. }\end{array}$ \\
\hline VT436 & $32-33 E$ & Bridging & Bridging about 12 way to peak between Col. 32E \& 33E. \\
\hline VT439 & 33-34E & Bridging & $\begin{array}{l}\text { Bridging about } 12 \text { way to the peak from wall between Col. } 33 E \& \\
34 E \text {. }\end{array}$ \\
\hline VT440 & 34-35E & Bridging & Bridging $\sim 12$ way to the peak from wall between Col. $34 \mathrm{E} \& 35 \mathrm{E}$. \\
\hline VT441 & $34 \mathrm{E}$ & Truss & Top of truss about 112 way to peak from Col. $34 \mathrm{E}$. \\
\hline VT442 & $35 \mathrm{E}$ & Truss & Top of truss about 12 way to the peak from Col. $35 \mathrm{E}$. \\
\hline VT443 & $35-36 \mathrm{E}$ & Bridging & $\begin{array}{l}\text { Bridging about } 12 \text { way to the peak from the wall between Col. } 35 \mathrm{E} \\
\& 36 \mathrm{E} \text {. Some dust is visible on the bridge in this location. }\end{array}$ \\
\hline VT444 & $36 \mathrm{E}$ & Truss & Top of truss about 12 way to the peak from Col. $36 \mathrm{E}$. \\
\hline VT445 & $36-37 \mathrm{E}$ & Bridging & Bridging about 12 way to peak from wall between Col. $36 \mathrm{E} \& 37 \mathrm{E}$. \\
\hline VT446 & $37 \mathrm{E}$ & Truss & Top of truss about 12 way to the peak from Col. $37 \mathrm{E}$. \\
\hline VT447 & $37-38 \mathrm{E}$ & Bridging & Bridging $\sim 12$ way to the peak from wall between Col. $37 E \& 38 E$. \\
\hline VT448 & $38 E$ & Truss & $\begin{array}{l}\text { Top of lower horizontal truss brace } \sim 12 \text { way to the peak from Col. } \\
38 \mathrm{E} \text {. }\end{array}$ \\
\hline
\end{tabular}


Table 5. (continued)

\begin{tabular}{|c|c|c|c|}
\hline $\begin{array}{c}\text { Sample } \\
\text { ID } a\end{array}$ & $\begin{array}{l}\text { Room/ } \\
\text { structure/ I } \\
\text { area }^{b}\end{array}$ & $\begin{array}{l}\text { Location } \\
\text { in bay }\end{array}$ & Location notesc \\
\hline VT449 & $38-39 E$ & Stringer & $\begin{array}{l}\text { Upper surface of the stringer about } 12 \text { way to peak from the wall } \\
\text { between Col. } 38 \mathrm{E} \& 39 \mathrm{E} \text {. }\end{array}$ \\
\hline VT450 & $38-39 E$ & Bridging & Bridgin $g \sim 12$ way to peak from the wall between Col. $38 \mathrm{E} \& 39 \mathrm{E}$. \\
\hline VT451 & 30-31E & Wall & Pre-smear beta-gamma \& alpha scans conducted. \\
\hline VT452 & $30-31 \mathrm{E}$ & Window & Pre-smear beta-gamma scan conducted. \\
\hline VT453 & 29-30E & Wall & Pre-smear beta-gamma \& alpha scans conducted. \\
\hline VT454 & 28-29E & Wall & Pre-smear beta-gamma scan conducted. \\
\hline VT456 & $27-28 \mathrm{E}$ & Wall & Pre-smear beta-gamma \& alpha scans conducted. \\
\hline VT457 & 26-27E & Wall & Pre-smear beta-gamma \& alpha scans conducted. \\
\hline VT460 & $25-26 E$ & Wall & Pre-smear beta-gamma \& alpha scans conducted. \\
\hline VT461 & $21-22 \mathrm{E}$ & Wall & This location is visibly dirty. Pre-smear beta-gamma scan conducted. \\
\hline VT462 & $21-22 \mathrm{E}$ & Other & $\begin{array}{l}\text { Top of lower header which is about } 3 \text { in. wide (this location was } \\
\text { selected as the upper window ledges between Col. } 21 \mathrm{E} \& 24 \mathrm{E} \text { are } \\
\text { too narrow to smear) between Col. } 21 \mathrm{E} \& 22 \mathrm{E} \text {. Pre-smear beta- } \\
\text { gamma scan conducted. }\end{array}$ \\
\hline VT463 & $22-23 E$ & Other & $\begin{array}{l}\text { Top of lower header which is about } 3 \text { in. wide (this location was } \\
\text { selected as the upper window ledges between Col. } 21 \mathrm{E} \& 24 \mathrm{E} \text { are } \\
\text { too narrow to smear) between Col. } 22 \mathrm{E} \& 23 \mathrm{E} \text {. Pre-smear beta- } \\
\text { gamma scan conducted. }\end{array}$ \\
\hline VT464 & $22-23 E$ & Wall & $\begin{array}{l}\text { Top of plate which is visibly dirty located between Col. } 22 \mathrm{E} \& 23 \mathrm{E} \text {. } \\
\text { Pre-smear beta-gamma scan conducted. }\end{array}$ \\
\hline VT465 & $23-24 \mathrm{E}$ & Wall & A beta-gamma \& alpha scan was done directly on the smear sample. \\
\hline VT467 & MS-1 & Wall & $\begin{array}{l}\text { Top of the wall in MS-1 (furnace room) about } 11 \mathrm{ft} \& 9 \text { in. off the } \\
\text { floor on the } S \text { side. Beta-gamma \& alpha scans were done directly } \\
\text { on the smear. }\end{array}$ \\
\hline VT468 & $23-24 W$ & Other & $\begin{array}{l}\text { Top of the lower header between Col. } 23 \mathrm{~W} \& 24 \mathrm{~W} \text {. Beta-gamma \& } \\
\text { alpha scans were conducted directly on the smear. }\end{array}$ \\
\hline VT469 & $23-24 W$ & Wall & $\begin{array}{l}\text { Top of a visibly very dirty plate between Col. } 23 \mathrm{~W} \& 24 \mathrm{~W} \text {. Beta- } \\
\text { gamma and alpha scans were conducted directly on the smear. }\end{array}$ \\
\hline VT470 & $23 \mathrm{~W}$ & Truss & Beta-gamma \& alpha scans conducted directly on the smear. \\
\hline VT471 & $28-29 \mathrm{~W}$ & Window & $\begin{array}{l}\text { Upper center window between Col. } 28 \mathrm{~W} \& 29 \mathrm{~W} \text {. Beta-gamma \& } \\
\text { alpha scans conducted directly on the smear. }\end{array}$ \\
\hline VT472 & $28-29 \mathrm{~W}$ & Wall & Beta-gamma \& alpha scans conducted directly on the smear. \\
\hline VT473 & $27-28 \mathrm{~W}$ & Window & $\begin{array}{l}\text { Upper center window ledge between Col. } 27 \mathrm{~W} \& 28 \mathrm{~W} \text {. Beta-gamma } \\
\& \text { alpha scans conducted directly on the smear. }\end{array}$ \\
\hline VT474 & $27-28 \mathrm{~W}$ & Wall & Beta-gamma \& alpha scans conducted directly on the smear. \\
\hline
\end{tabular}


Table 5. (continued)

\begin{tabular}{|c|c|c|c|}
\hline $\begin{array}{l}\text { Sample } \\
\text { ID } a\end{array}$ & $\begin{array}{l}\text { Room/ } \\
\text { structure/ Loc } \\
\text { area }^{b} \quad \text { in }\end{array}$ & & Location notesc \\
\hline VT475 & $26-27 \mathrm{~W}$ & Window & $\begin{array}{l}\text { Upper center window ledge between Col. } 26 \mathrm{~W} \& 27 \mathrm{~W} \text {. Beta-gamma } \\
\& \text { alpha scans conducted directly on the smear. }\end{array}$ \\
\hline VT476 & $26-27 \mathrm{~W}$ & Wall & Beta-gamma \& alpha scans conducted directly on the smear. \\
\hline VT477 & $29-30 \mathrm{~W}$ & Window & $\begin{array}{l}\text { Upper south window ledge between Col. } 29 \mathrm{~W} \& 30 \mathrm{~W} \text {. Beta-gamma } \\
\text { and alpha scans conducted directly on the smear. }\end{array}$ \\
\hline VT478 & $29-30 \mathrm{~W}$ & Wall & Beta-gamma \& alpha scans conducted directly on the smear. \\
\hline VT479 & $30-31 \mathrm{~W}$ & Window & $\begin{array}{l}\text { Upper middle window ledge between Col. } 30 \mathrm{~W} \& 31 \mathrm{~W} \text {. Beta- } \\
\text { gamma \& alpha scans conducted directly on the smear. }\end{array}$ \\
\hline VT481 & $39-40 \mathrm{~W}$ & Wall & Top of concrete block wall between Col. $39 \mathrm{~W} \& 40 \mathrm{~W}$. \\
\hline VT486 & $41 \mathrm{E}$ & Truss & $\begin{array}{l}\text { Top of lower horizontal truss member about } 12 \text { way to the peak } \\
\text { from Col. } 41 \mathrm{E} \text {. }\end{array}$ \\
\hline VT490 & $42-43 E$ & Stringer & $\begin{array}{l}\text { Upper surface of stringer about } 12 \text { way to peak from wall between } \\
\text { Col. } 42 \mathrm{E} \& 43 \mathrm{E} \text {. }\end{array}$ \\
\hline VT491 & $42-43 E$ & Bridging & Bridging about 12 way to peak from wall between Col. $42 \mathrm{E} \& 43 \mathrm{E}$. \\
\hline VT492 & NE bldg. & Wall & $\begin{array}{l}\text { Top of wood plate (above block wall) about } 16 \mathrm{ft} \mathrm{W} \text { of the NE corner } \\
\text { of the building. }\end{array}$ \\
\hline VT493 & $43 \mathrm{E}$ & Bridging & $\begin{array}{l}\text { Bridging about } 12 \text { way to the peak from wall between Col. } 43 E \& \\
\text { NE corner of the building. }\end{array}$ \\
\hline VT494 & $43 \mathrm{E}$ & Truss & Top of truss $\sim 1 \mathrm{ft}$ east of the roof peak on Column line $43 \mathrm{E}-\mathrm{W}$. \\
\hline VT495 & $39 \mathrm{E}$ & Truss & Top of truss about 12 way to peak from Col. $39 \mathrm{E}$. \\
\hline VT496 & $39-40 \mathrm{E}$ & Bridging & Bridging about 12 way to peak from wall between Col $39 \mathrm{E} \& 40 \mathrm{E}$. \\
\hline VT497 & $39-40 \mathrm{~W}$ & Bridging & Bridging $\sim 13$ way to peak from wall between Col $39 \mathrm{~W} \& 40 \mathrm{~W}$. \\
\hline VT498 & $39 \mathrm{~W}$ & Truss & Top of truss about 12 way to peak from Col. $39 \mathrm{~W}$ \\
\hline VT499 & $39-40 \mathrm{~W}$ & Stringer & $\begin{array}{l}\text { Upper vertical surface on stringer about } 13 \text { way to peak from wall } \\
\text { between Cols. } 39 \mathrm{~W} \& 40 \mathrm{~W} \text {. }\end{array}$ \\
\hline VT500 & $30-31 \mathrm{E}$ & Bridging & Pre-smear alpha and beta-gamma scans conducted. \\
\hline VT501 & 30-31W & Wall & Beta-gamma \& alpha scans conducted directly on the smear. \\
\hline VT515 & $21 W-22 W$ & Wall & Pre-smear beta-gamma scan conducted. \\
\hline VT517 & $29-30 \mathrm{E}$ & Bridging & Bridging about 12 way to peak from wall between Col. $29 \mathrm{E} \& 30 \mathrm{E}$. \\
\hline VT520 & $31 \mathrm{~W}$ & Truss & Top of truss about 12 way to peak from Col. $31 \mathrm{~W}$. \\
\hline VT521 & $30-31 \mathrm{~W}$ & Bridging & Bridging $\sim 12$ way to peak from wall between Col. $30 \mathrm{~W} \& 31 \mathrm{~W}$. \\
\hline VT526 & $39 \mathrm{~W}$ & Truss & $\begin{array}{l}\text { Top of truss about } 2 \mathrm{ft} \text { away from peak at Col, } 39 \mathrm{~W} \text {. This sample } \\
\text { was taken after additional cleaning. }\end{array}$ \\
\hline VT527 & $22-23 \mathrm{E}$ & Bridging & $\begin{array}{l}\text { This location is the metal shop south wall. "None detectable" on the } \\
\text { beta-gamma\& alpha pre-smear scans. }\end{array}$ \\
\hline
\end{tabular}


Table 5. (continued)

\begin{tabular}{|c|c|c|c|}
\hline $\begin{array}{c}\text { Sample } \\
\text { ID } a\end{array}$ & $\begin{array}{l}\text { Room/ } \\
\text { structure/ I } \\
\text { area }^{b}\end{array}$ & $\begin{array}{c}\text { Location } \\
\text { in bay }\end{array}$ & Location notesc \\
\hline VT528 & $22 \mathrm{E}$ & Truss & "None detectable" on the pre-smear beta-gamma \& alpha scans. \\
\hline VT529 & 22-23E & Stringer & $\begin{array}{l}\text { This location is the metal shop south wall. The upper vertical surface } \\
\text { of the stringer was smeared. "None detectable" on the pre-smear } \\
\text { beta-gamma \& alpha scans. }\end{array}$ \\
\hline VT530 & $22-23 E$ & Bridging & "None detectable" on the beta-gamma \& alpha pre-smear scans. \\
\hline VT535 & $28-29 \mathrm{E}$ & Stringer & This location is inside the ceiling ventilation shaft. \\
\hline VT541 & $26 \mathrm{~W}$ & Truss & Pre-smear alpha and beta-gamma scans were conducted. \\
\hline VT542 & $25-26 \mathrm{~W}$ & Bridging & Pre-smear alpha and beta-gamma scans were conducted. \\
\hline VT543 & $25-26 \mathrm{E}$ & Bridging & Pre-smear alpha and beta-gamma scans were conducted. \\
\hline VT544 & $25 \mathrm{E}$ & Truss & Pre-smear alpha \& beta-gamma scans conducted. \\
\hline VT545 & $24-25 \mathrm{E}$ & Bridging & Pre-smear alpha and beta-gamma scans conducted. \\
\hline VT546 & $25 \mathrm{~W}$ & Truss & Pre-smear alpha and beta-gamma scans conducted. \\
\hline VT547 & $24-25 \mathrm{~W}$ & Bridging & Pre-smear alpha and beta-gamma scans conducted. \\
\hline VT548 & $24 \mathrm{E}$ & Wall & Pre-smear alpha and beta-gamma scans conducted. \\
\hline VT549 & $24 \mathrm{~W}$ & Truss & $\begin{array}{l}\text { Top of truss at roofline step-down between metal shop and carpenter } \\
\text { end and about } 4 \mathrm{ft} \text { from peak to Col. line } 24 \mathrm{~W} \text {. Pre-smear alpha and } \\
\text { beta-gamma scans conducted. }\end{array}$ \\
\hline VT551 & $23-24 \mathrm{E}$ & Bridging & Pre-smear alpha and beta-gamma scans conducted. \\
\hline VT552 & $23 \mathrm{~W}$ & Truss & Pre-smear alpha and beta-gamma scans conducted. \\
\hline VT553 & $22-23 W$ & Bridging & Pre-smear alpha and beta-gamma scans conducted. \\
\hline VT554 & $22 \mathrm{~W}$ & Truss & Pre-smear alpha and beta-gamma scans conducted. \\
\hline VT555 & $21-22 W$ & Bridging & $\begin{array}{l}\text { The location is on the metal-shop side of block wall. Pre-smear alpha } \\
\text { and beta-gamma scans conducted. }\end{array}$ \\
\hline VT556 & $31 \mathrm{~W}$ & Floor & $\begin{array}{l}\text { Crane rail track located on the floor about } 7 \mathrm{ft} E \& 4 \mathrm{ft} N \text { of Col. } \\
31 \mathrm{~W} \text {. This sample was taken at the old location of VM } 443 \text { after } \\
\text { cleaning. }\end{array}$ \\
\hline VT557 & $42 W$ & Floor & $\begin{array}{l}\text { Crane rail track on the floor } 7 \mathrm{ft} \text { directly east of Col. } 42 \mathrm{~W} \text {. This } \\
\text { sample was taken after extensive additional cleaning. }\end{array}$ \\
\hline VT559 & $38 \mathrm{~W}$ & Floor & $\begin{array}{l}\text { This location is the same as old VM } 445 \text { and sample VT559 was } \\
\text { taken after extensive additional cleaning. }\end{array}$ \\
\hline VT560 & $27 \mathrm{E}$ & Floor & Sample was taken after extensive additional cleaning. \\
\hline VT561 & $22 \mathrm{~W}$ & Other & $\begin{array}{l}\text { Upper horizontal surface of original (?) electrical fuse box located } 8 \\
\mathrm{ft} \text { above floor level and } 33 \mathrm{ft} \text { directly } \mathrm{S} \text { of Col.22W. This item and } \\
\text { area are above the false ceiling. }\end{array}$ \\
\hline VT565 & $15 \mathrm{~W}$ & Other & $\begin{array}{l}\text { Upper horizontal surface of original (?) electrical fuse box located } 5 \\
\mathrm{ft} \text { above floor level and } 1 \mathrm{ft} \mathrm{N} \text { of Col. } 15 \mathrm{~W} \text {. }\end{array}$ \\
\hline
\end{tabular}


Table 5. (continued)

\begin{tabular}{|c|c|c|c|}
\hline $\begin{array}{l}\text { Sample } \\
\text { IDa }\end{array}$ & $\begin{array}{l}\text { Room/ } \\
\text { structure/ } \\
\text { areab }^{b}\end{array}$ & $\begin{array}{l}\text { Location } \\
\text { in bay }\end{array}$ & Location notesc \\
\hline VT574 & $18-19 E$ & Bridging & Between stringers $8 \& 9$ \\
\hline VT575 & $18-19 \mathrm{E}$ & Truss & Between stringers $14 \& 15$ \\
\hline VT577 & $17-18 \mathrm{E}$ & Bridging & Between stringers $8 \& 9$ \\
\hline VT580 & $16-17 \mathrm{E}$ & Bridging & Between stringers $8 \& 9$ \\
\hline VT582 & $8-9 W$ & Wall & $\begin{array}{l}\text { Bay ID:Pie-shaped area between columns at S. end of Bldg. } S \text { of } 8- \\
9 W .\end{array}$ \\
\hline VT583 & $8-9 W$ & Wall & This location is the same as top of block wall. \\
\hline VT586 & $8 W$ & Truss & $\begin{array}{l}\text { This location is the pie-shaped section at south end of Bldg. south of } \\
8-9 \mathrm{~W} \text {. }\end{array}$ \\
\hline VT587 & $9-10 \mathrm{~W}$ & Truss & This location is the same as the top of block wall. \\
\hline VT590 & $10-11 \mathrm{~W}$ & Truss & This location is the same as top of the block wall. \\
\hline VT597 & $8 \mathrm{E}$ & Wall & $\begin{array}{l}\text { Top of block wall (wood plate), W of Col. } 8 \mathrm{E} \text { (SE corner), about } 2 \backslash 3 \\
\text { way toward truss center \& about } 4 \mathrm{ft} \text { W of old VM370. }\end{array}$ \\
\hline VT654 & $7 S$ & Truss & $\begin{array}{l}\text { Support beam functions like top of block wall structure where } \\
\text { building turns west at south end of this location. }\end{array}$ \\
\hline VT655 & $7 S$ & Stringer & No bridging is present on $S$ side of this triangular bay. \\
\hline VT729 & $1 \mathrm{~N}$ & Wall & This location is near Col. $1 \mathrm{~N}$. \\
\hline VT732 & $1 \mathrm{~N}$ & Wall & This location is near Col. $1 \mathrm{~N}$. \\
\hline VT782 & $41-42 W$ & Bridging & $\begin{array}{l}\text { This sample from the bridging was taken (before additional } \\
\text { recleaning) in the room north of the refrigeration room and } 10 \mathrm{ft} \mathrm{W} \\
\& 10 \mathrm{ft} \mathrm{S} \mathrm{of} \mathrm{Col.} 42 \text {. }\end{array}$ \\
\hline VT788 & MS-1 & Bridging & This location is above the roof trusses. \\
\hline VT790 & $26-27 \mathrm{~W}$ & Drain & VT789 was taken at this location for rad analysis. \\
\hline VT797 & $38 \mathrm{~W}$ & Floor & Location is $9.5 \mathrm{ft} \mathrm{W}$ and $10 \mathrm{ft} \mathrm{N}$ of Col. $38 \mathrm{~W}$. \\
\hline VT799 & 26-27E & Stringer & $\begin{array}{l}\text { This sample taken one ft } \mathrm{N} \text { of old VT } 458 \text { after additional cleaning } \\
\text { had been conducted. This stringer is directly over the east wall. }\end{array}$ \\
\hline VT801 & 20-21E & Wall & $\begin{array}{l}\text { This sample was taken directly N of old VT567 after additional } \\
\text { cleaning. }\end{array}$ \\
\hline VT823 & $27 \mathrm{~W}$ & Wall & $\begin{array}{l}\text { Boiler room sand box wall } 15 \mathrm{ft} \text { due } \mathrm{N} \text { of Col. } 27 \mathrm{~W} \text {. The location is } \\
7 \mathrm{ft} \text { and } 3 \mathrm{in} \text {. above the high bay floor level and } 1 \mathrm{ft} \text { below the } \\
\text { original sand level. This is the same wall as VM456. }\end{array}$ \\
\hline VT825 & $27 \mathrm{~W}$ & Wall & $\begin{array}{l}\text { Wall location is } 15 \mathrm{ft} \text { due } \mathrm{N} \text { of } \mathrm{Col} 27 \mathrm{~W} ; 11 \mathrm{ft} \& 3 \mathrm{in} \text {. above the bay } \\
\text { floor level; and about } 3 \mathrm{ft} \text { above the original sand level. }\end{array}$ \\
\hline VT827 & $27 \mathrm{~W}$ & Wall & $\begin{array}{l}\text { Boiler room sand box north wall at } 18 \mathrm{ft} \& 6 \text { in. } \mathrm{N} \text { and } 10 \mathrm{ft} \mathrm{W} \text { of } \\
\text { Col. } 27 \mathrm{~W} \text {. The location is } 7 \mathrm{ft} \text { and } 3 \mathrm{in} \text {. above the bay floor level and } \\
\text { about } 1 \mathrm{ft} \text { below the original sand level. }\end{array}$ \\
\hline
\end{tabular}


Table 5. (continued)

\begin{tabular}{|c|c|c|c|}
\hline $\begin{array}{c}\text { Sample } \\
\text { ID } a\end{array}$ & $\begin{array}{l}\text { Room/ } \\
\text { structure/ L } \\
\text { area }^{b}\end{array}$ & $\begin{array}{l}\text { Location } \\
\text { in bay }\end{array}$ & Location notesc \\
\hline VT829 & $27 \mathrm{~W}$ & Wall & $\begin{array}{l}\text { Boiler room sand box north wall at } 18 \mathrm{ft} \& 6 \mathrm{in} . \mathrm{N} \text { and } 10 \mathrm{ft} \mathrm{W} \text { of } \\
\text { Col. } 27 \mathrm{~W} \text {. This location is } 11 \mathrm{ft} \& 3 \mathrm{in} \text {. above the bay floor level and } \\
\text { about } 3 \mathrm{ft} \text { above the original sand level. }\end{array}$ \\
\hline VT830 & $27 \mathrm{~W}$ & Wall & $\begin{array}{l}\text { Boiler room sand box west wall at } 10 \mathrm{ft} \& 6 \mathrm{in} . \mathrm{N} \text { and } 19 \mathrm{ft} \& 1 \mathrm{in} \text {. } \\
\mathrm{W} \text { of Col. } 27 \mathrm{~W} \text {. This location is } 7 \mathrm{ft} \& 3 \text { in.above bay floor level and } \\
\text { about } 1 \mathrm{ft} \text { below the original sand level. }\end{array}$ \\
\hline VT831 & $27 \mathrm{~W}$ & Wall & $\begin{array}{l}\text { This location is } 10 \mathrm{ft} \& 3 \mathrm{in} \text {. above the bay floor level and about } 2 \mathrm{ft} \\
\text { above the original sand level. }\end{array}$ \\
\hline VT863 & $2 S$ & Drain & $\begin{array}{l}\text { This location is a } 4 \text {-in. diameter cast iron drain running in a NW-SE } \\
\text { direction. The beta-gamma scan inside the pipe was N.D. above } \\
\text { background. This sample is a resmear of VT } 840 \text { in the wood shop } \\
\text { area. }\end{array}$ \\
\hline VT864 & $2 N$ & Drain & $\begin{array}{l}\text { This } 2 \text {-in. diameter cast iron drain pipe was scanned for beta-gamma } \\
(24 \mathrm{cpm} \text { above } B G=57 \mathrm{cpm}=0.03 \mathrm{mrad} / \mathrm{h}) \text { at the opening and } \\
\text { smeared for Be to a vertical depth of } 6 \mathrm{in} \text {. This sample is a resmear } \\
\text { of VT842 in the wood shop area. }\end{array}$ \\
\hline VT865 & Drain 57 & Drain & $\begin{array}{l}\text { This 3-in. diameter cast iron Drain } 57 \text { pipe was scanned for beta- } \\
\text { gamma (N.D.above BG) inside pipe and smeared for Be to a vertical } \\
\text { depth of } 8 \text { in. This sample supplements VM } 481 \text { taken in the wood } \\
\text { shop area. }\end{array}$ \\
\hline VT866 & Drain 56 & Drain & $\begin{array}{l}\text { This 2-in. diameter cast iron Drain } 56 \text { pipe was scanned for beta- } \\
\text { gamma (N.D above BG) at the opening and smeared for Be to a } \\
\text { vertical depth of } 12 \text { in. This sample is a resmear of VT } 846 \text { \& VT } 848 \\
\text { in the wood shop area. VT } 986 \text { was recheck. }\end{array}$ \\
\hline VT867 & Drain 55 & Drain & $\begin{array}{l}\text { This } 3 \text { in. diameter cast iron Drain } 55 \text { pipe was scanned for beta- } \\
\text { gamma (N.D.above BG) at the Drain opening and smeared for Be to } \\
\text { a vertical depth of } 8 \text { in. This sample is a resmear of VT } 850 \text { in the } \\
\text { wood shop area. }\end{array}$ \\
\hline VT868 & Drain 54 & Drain & $\begin{array}{l}\text { This } 3 \text { in. diameter cast iron Drain } 54 \text { pipe was scanned for beta- } \\
\text { gamma (N.D.above BG) at the Drain opening and smeared for Be to } \\
\text { a vertical depth of } 8 \text { in. This sample is a resmear of VT } 852 \text { in the } \\
\text { wood shop area. }\end{array}$ \\
\hline VT870 & $27 E$ & Drain & $\begin{array}{l}\text { This 4-in. diam. cast clay pipe was scanned for beta-gamma } \\
\text { (N.D.above BG) at the Drain opening and inside the pipe and was } \\
\text { smeared for Be to a vertical depth of } 6 \text { in. This sample is a resmear } \\
\text { of VT861 in the metal shop area. }\end{array}$ \\
\hline VT871 & $27 \mathrm{E}$ & Drain & $\begin{array}{l}\text { This } 4 \text { in. diameter cast clay Drain pipe was scanned for beta-gamma } \\
\text { (N.D. above BG) at the Drain opening and in the pipe and smeared } \\
\text { for Be to a vertical depth of } 3 \text { in. (there was a solid obstruction in the } \\
\text { pipe at } 3 \text { in. depth.) }\end{array}$ \\
\hline VT872 & $28 \mathrm{E}$ & Truss & Beta-gamma scan N.D. above background ( $37 \mathrm{cpm}=0.02 \mathrm{mrad} / \mathrm{h}$ ). \\
\hline VT873 & $28 \mathrm{E}$ & Truss & Beta-gamma scan N.D. above BG (37 cpm $=0.02 \mathrm{mrad} / \mathrm{h})$. \\
\hline
\end{tabular}


Table 5. (continued)

\begin{tabular}{|c|c|c|c|}
\hline $\begin{array}{l}\text { Sample } \\
\text { ID } a\end{array}$ & $\begin{array}{l}\text { Room/ } \\
\text { structure/ I } \\
\text { area }^{b}\end{array}$ & $\begin{array}{l}\text { Location } \\
\text { in bay }\end{array}$ & Location notesc \\
\hline VT874 & $25-26 \mathrm{~W}$ & Bridging & Beta-gamma scan N.D. above BG $(37 \mathrm{cpm}=0.02 \mathrm{mrad} / \mathrm{h})$ \\
\hline VT875 & $24-25 \mathrm{~W}$ & Window & Beta-gamma scan N.D. above BG $(37 \mathrm{cpm}=0.02 \mathrm{mrad} / \mathrm{h})$. \\
\hline VT876 & $24-25 \mathrm{E}$ & Wall & Beta-gamma scan N.D. above BG $(37 \mathrm{cpm}=0.02 \mathrm{mrad} / \mathrm{h})$. \\
\hline VT877 & $23-24 \mathrm{~W}$ & Bridging & $\begin{array}{l}\text { Beta-gamma scan was N.D. above BG }(37 \mathrm{cpm}=0.02 \mathrm{mrad} / \mathrm{h}) \text {. } \\
\text { VT788 was also taken from this bay. }\end{array}$ \\
\hline VT878 & 23-24E & Other & $\begin{array}{l}\text { Beta-gamma scan N.D. above BG }(37 \mathrm{cpm}=0.02 \mathrm{mrad} / \mathrm{h}) . \mathrm{VT} 466 \\
\text { was also taken in this bay. }\end{array}$ \\
\hline VT881 & $26 \mathrm{~W}$ & Drain & $\begin{array}{l}\text { This } 4 \text { in. cast iron Drain line runs } N \text { to } S \text { with the opening being } \\
\text { about } 4 \mathrm{ft} \text { below floor level. Logged for radon } 12-11-93 \text {. The beta- } \\
\text { gamma scan was N.D. above BG }(37 \mathrm{cpm}=0.02 \mathrm{mrad} / \mathrm{h}) \text {. Sample } \\
\text { VT } 882 \text { was taken at this same location for rad analysis as was VM5 }\end{array}$ \\
\hline VT885 & $22 \mathrm{~W}$ & Floor & $\begin{array}{l}\text { Old floor (? original) in west closet area underneath raised floor in } \\
\text { NE comer of closet at old H. P. office which is } \mathrm{W} \text { of Col. } 22 \mathrm{~W} \text {. The } \\
\text { beta gamma scan range for the entire closet floor }=55-85 \mathrm{cpm} \text { ( = } \\
0.03-0.04 \mathrm{mrad} / \mathrm{h} \text { ).VT } 886 \text { was taken very near this area for rad } \\
\text { analysis }\end{array}$ \\
\hline VT887 & $22 \mathrm{~W}$ & Wall & $\begin{array}{l}\text { Smear taken from the first block face from the floor of the old H.P. } \\
\text { office (west of Col. } 22 \mathrm{~W} \text { ) north wall about } 7 \mathrm{ft} \text { from the NE corner. } \\
\text { VT888 was taken very near this location for rad analysis. }\end{array}$ \\
\hline VT889 & $22 \mathrm{~W}$ & Floor & $\begin{array}{l}\text { Location is about } 6 \text { in. W and } 6 \text { in. } S \text { of corner joints. VT } 890 \text { was } \\
\text { sampled very near this area for rad analysis. }\end{array}$ \\
\hline VT891 & $22 \mathrm{~W}$ & Other & $\begin{array}{l}\text { The old health physics office is west of Col. } 22 \mathrm{~W} \text {. Pipe is } 1.5 \mathrm{in} \text {. } \\
\text { diameter and is running north/south. The beta-gamma scan was N.D. } \\
\text { above BG ( } 40 \mathrm{cpm}=0.02 \mathrm{mrad} / \mathrm{h}) \text { at this location. }\end{array}$ \\
\hline VT892 & $24 \mathrm{~W}$ & Drain & $\begin{array}{l}\text { Smear taken to a } 6 \text { in. vertical depth from a } 4 \text { in. diameter clay pipe } \\
\text { (one of two pipes leading to pit) entering a pit. This pipe is } 54 \text { in. } \\
\text { deep to the elbow, turning SE } 7.5 \mathrm{ft} S \text { and } 3 \mathrm{ft} E \text { of Col. } 24 \mathrm{~W} \text { on the } \\
\text { outside wall of MS-1. The beta-gamma scan was N.D. above } \\
\text { background. }\end{array}$ \\
\hline VT894 & $24 \mathrm{~W}$ & Drain & $\begin{array}{l}\text { Smear (to a vertical depth of } 6 \mathrm{in} \text {.) of a } 4 \mathrm{in} \text {. diameter clay drain pipe } \\
\text { (one of } 2 \text { pipes entering the pit) to a pit at } 7.5 \mathrm{ft} S \text { and } 3 \mathrm{ft} E \text { of Col. } \\
24 \mathrm{~W} \text {. This drain is } 30 \mathrm{in} \text {. deep to the elbow,turns SE, and is located } \\
\text { at the outside wall of MS-1. Smear taken to a depth of 6-9 in. }\end{array}$ \\
\hline VT896 & $24 \mathrm{~W}$ & Drain & $\begin{array}{l}\text { Small ( } 1.5 \text { in. diameter) black iron (possibly galvanized) drain pipe } \\
\text { smeared to a vertical depth of } 10 \text { inches. This pipe is } 10 \text { in. deep to } \\
\text { the elbow; turns due E; connects to the galvanized floor Drain of } \\
\text { location VT } 897 \text {; and is located } 2 \mathrm{ft} N \& 2.25 \mathrm{ft} E \text { of Col. } 24 \mathrm{~W} \\
\text { (extreme W wall). }\end{array}$ \\
\hline
\end{tabular}


Table 5. (continued)

\begin{tabular}{|c|c|c|c|}
\hline $\begin{array}{l}\text { Sample } \\
\text { ID } a\end{array}$ & $\begin{array}{c}\text { Room/ } \\
\text { structure/ } \\
\text { area }^{b}\end{array}$ & $\begin{array}{c}\text { Location } \\
\text { in bay }\end{array}$ & Location notesc \\
\hline VT900 & $26 \mathrm{~W}$ & Drain & $\begin{array}{l}\text { 3-in. diameter clay-ceramic drain pipe which is } 57 \text { in. deep with } \\
\text { liquid in "T" at } 57 \text { in. depth. Ref. KAPL } 38 \text { rad scan to } 3 \mathrm{ft} \text { depth. } \\
\text { See sample VT901 for the rad sample taken at this location at } 8-11 \\
\text { in. depth. }\end{array}$ \\
\hline VT902 & $28 \mathrm{~W}$ & Drain & $\begin{array}{l}\text { 4-in. diameter clay-ceramic drain pipe } 17 \text { in. deep to elbow tuming } \\
\text { south. Ref. KAPL } 43 \text { rad scan to depth of } 17 \text { in. See VT } 903 \text { for the } \\
\text { rad sample taken at the } 6-9 \text { in. depth of this location. }\end{array}$ \\
\hline VT904 & $29 \mathrm{~W}$ & Drain & $\begin{array}{l}4 \text { in. diameter black iron pipe } 41 \text { in.deep to elbow turning south. Rad } \\
\text { scan taken to a depth of } 41 \text { in. See sample VT905 for the rad smear } \\
\text { taken taken at the } 6-9 \text { in. depth of this location. }\end{array}$ \\
\hline VT906 & $30 \mathrm{~W}$ & Drain & $\begin{array}{l}2.5 \text { in.diameter cast iron pipe connecting to "T" at } 14 \text { in. depth east } \\
\text { to west. Ref.KAPL } 45 \text { for rad scan to a depth of } 14 \text { in. Connects to } \\
\text { VM508 \& VT } 884 \text { in west direction. Sample VT907 taken at this } \\
\text { location (10-12 in. depth) for rad analysis. }\end{array}$ \\
\hline VT908 & $31 \mathrm{~W}$ & Drain & $\begin{array}{l}4 \text { in. diameter cast iron drain pipe } 32 \text { in. deep to elbow turning south. } \\
\text { Ref. KAPL } 59 \text {. Sample VT909 taken at this location at } 6-9 \text { inch } \\
\text { depth for rad analysis. }\end{array}$ \\
\hline VT911 & $37 \mathrm{~W}$ & Drain & $\begin{array}{l}\text { This sample is a supplement to VM499. SeeVT912 for the rad } \\
\text { sample at this location. }\end{array}$ \\
\hline VT913 & $2 \mathrm{~N}$ & Other & Beta-gamma scan was N.D. above BG $(51 \mathrm{cpm}=0.03 \mathrm{mrad} / \mathrm{h})$. \\
\hline VT914 & $2 \mathrm{~N}$ & Window & Beta-gamma scan was N.D. above BG $(51 \mathrm{cpm}=0.03 \mathrm{mrad} / \mathrm{h})$. \\
\hline VT915 & $3 N$ & Other & Beta-gamma scan was N.D. above $B G(51 \mathrm{cpm}=0.03 \mathrm{mrad} / \mathrm{h})$. \\
\hline VT916 & $2 \mathrm{~N}$ & Floor & $\begin{array}{l}\text { VT917 is the rad analysis sample taken in this area ( } 1 \mathrm{ft} \text { east). The } \\
\text { beta-gamma scan was N.D. above BG }(44 \mathrm{cpm}=0.02 \mathrm{mrad} / \mathrm{h}) \text {. }\end{array}$ \\
\hline VT918 & $3 \mathrm{~N}$ & Floor & $\begin{array}{l}\text { VT919 is the rad analysis sample taken in this area (one } \mathrm{ft} \text { east). The } \\
\text { beta-gamma scan was N.D. above BG }(44 \mathrm{cpm}=0.02 \mathrm{mrad} / \mathrm{h}) \text {. }\end{array}$ \\
\hline VT920 & $3 \mathrm{~N}$ & Floor & $\begin{array}{l}\text { VT921 is the sample taken for rad analysis near this location }(1 \mathrm{ft} \\
\text { west). The beta-gamma scan was N.D.above BG }(44 \mathrm{cpm}= \\
0.02 \mathrm{mrad} / \mathrm{h}) \text {. }\end{array}$ \\
\hline VT922 & $4 N$ & Floor & $\begin{array}{l}\text { VT923 was the sample taken for rad analysis in this area ( } 1 \mathrm{ft} \text { east). } \\
\text { The beta-gamma scan was N.D. above BG ( } 44 \mathrm{cpm}=0.02 \mathrm{mrad} / \mathrm{h}) \text {. }\end{array}$ \\
\hline VT924 & $5 \mathrm{~N}$ & Floor & $\begin{array}{l}\text { T932 is the sample taken for rad analysis in this area ( } 1 \mathrm{ft} \text { north). } \\
\text { The beta-gamma scan was N.D. above } B G(44 \mathrm{cpm}=0.02 \mathrm{mrad} / \mathrm{h}) \text {. }\end{array}$ \\
\hline VT925 & $3 \mathrm{~N}$ & Window & Beta-gamma scan was N.D. above BG $(5 i \mathrm{cpm}=0.03 \mathrm{mrad} / \mathrm{h})$. \\
\hline VT926 & $4 N$ & Other & $\begin{array}{l}\text { This location is just below the roof decking where the ceiling vent } \\
\text { had been removed. The beta-gamma scan was N.D. above BG ( } 51 \\
\mathrm{cpm}=0.03 \mathrm{mrad} / \mathrm{h} \text { ). }\end{array}$ \\
\hline VT927 & $4 \mathrm{~N}$ & Wall & $\begin{array}{l}\text { This location is } 6 \mathrm{ft} \text { above the floor level. The beta-gamma scan was } \\
\text { N.D. above } B G(51 \mathrm{cpm}=0.03 \mathrm{mrad} / \mathrm{h}) \text {. }\end{array}$ \\
\hline
\end{tabular}


Table 5. (continued)

\begin{tabular}{|c|c|c|c|}
\hline $\begin{array}{l}\text { Sample } \\
\text { ID } a\end{array}$ & $\begin{array}{l}\text { Room/ } \\
\text { structure/ I } \\
\text { area }^{b}\end{array}$ & $\begin{array}{l}\text { Location } \\
\text { in bay }\end{array}$ & Location notesc \\
\hline VT928 & $5 \mathrm{~N}$ & Other & The beta-gamma scan was N.D. above BG $(51 \mathrm{cpm}=0.03 \mathrm{mrad} / \mathrm{h})$. \\
\hline VT929 & $5 \mathrm{~N}$ & Window & Beta-gamma scan was N.D. above $B G(51 \mathrm{cpm}=0.03 \mathrm{mrad} / \mathrm{h})$. \\
\hline VT930 & $6 \mathrm{~N}$ & Other & $\begin{array}{l}\text { This location is equal to top of lower truss member; Beta-gamma } \\
\text { scan was N.D. above } B G(51 \mathrm{cpm}=0.03 \mathrm{mrad} / \mathrm{h}) \text {. }\end{array}$ \\
\hline VT931 & $6 \mathrm{~N}$ & Other & $\begin{array}{l}\text { This location is just below the ceiling. The beta-gamma scan is N.D. } \\
\text { above } B G(51 \mathrm{cpm}=0.03 \mathrm{mrad} / \mathrm{h}) \text {. }\end{array}$ \\
\hline VT933 & $6 \mathrm{~N}$ & Floor & $\begin{array}{l}\text { VT934 was the sample taken for rad analysis near this location (one } \\
\mathrm{ft} \text { north). The beta-gamma scan was N.D. above BG ( } 44 \mathrm{cpm}= \\
0.02 \mathrm{mrad} / \mathrm{h}) \text {. }\end{array}$ \\
\hline VT935 & $7 \mathrm{~N}$ & Floor & $\begin{array}{l}\text { VT936 was the sample taken for rad analysis near this location (one } \\
\mathrm{ft} \text { east). The beta-gamma scan was N.D.above BG }(44 \mathrm{cpm}= \\
0.02 \mathrm{mrad} / \mathrm{h})\end{array}$ \\
\hline VT937 & $7 \mathrm{~N}$ & Floor & $\begin{array}{l}\text { The beta-gamma scan was N.D. above BG }(46 \mathrm{cpm}=0.02 \mathrm{mrad} / \mathrm{h}) \text {. } \\
\text { VT938 is the sample for rad analysis taken very near this location. }\end{array}$ \\
\hline VT939 & $7 \mathrm{~N}$ & Floor & $\begin{array}{l}\text { This carpet area was under brooms, shovels, etc. and } 4 \mathrm{ft} \text { north of } \\
\text { VT937. The beta-gamma scan was N.D. above BG }(46 \mathrm{cpm}= \\
0.02 \mathrm{mrad} / \mathrm{h}) \text {. }\end{array}$ \\
\hline VT941 & $7 \mathrm{~N}$ & Other & $\begin{array}{l}\text { This location is just below the ceiling. The beta-gamma scan is N.D. } \\
\text { above BG }(51 \mathrm{cpm}=0.03 \mathrm{mrad} / \mathrm{h}) \text {. }\end{array}$ \\
\hline VT942 & $7 \mathrm{~N}$ & Other & $\begin{array}{l}\text { This location is just below the original ceiling. The beta-gamma scan } \\
\text { was N.D. above } B G(51 \mathrm{cpm}=0.03 \mathrm{mrad} / \mathrm{h}) \text {. }\end{array}$ \\
\hline VT943 & $13 \mathrm{~W}$ & Other & $\begin{array}{l}\text { This location is just below the original ceiling, but above the false } \\
\text { ceiling. The beta-gamma scan was N.D. above } B G(51 \mathrm{cpm}=0.03 \\
\mathrm{mrad} / \mathrm{h}) \text {. }\end{array}$ \\
\hline VT944 & $13 \mathrm{~W}$ & Other & $\begin{array}{l}\text { This location is just below the original ceiling. The beta-gamma scan } \\
\text { is N.D. above } B G(51 \mathrm{cpm}=0.03 \mathrm{mrad} / \mathrm{h}) \text {. }\end{array}$ \\
\hline VT945 & $13 \mathrm{~W}$ & Floor & $\begin{array}{l}\text { This location is near VT } 940 \text { (rad analysis) and the beta-gamma scan } \\
\text { was N.D. above BG ( } 46 \mathrm{cpm}=0.02 \mathrm{mrad} / \mathrm{h}) \text {. }\end{array}$ \\
\hline VT946 & $13 \mathrm{~W}$ & Floor & $\begin{array}{l}\text { This location is very near VT947 (rad analysis) and the beta-gamma } \\
\text { scan was N.D. above BG ( } 46 \mathrm{cpm}=0.02 \mathrm{mrad} / \mathrm{h} \text { ). }\end{array}$ \\
\hline VT953 & Stack & Wall & This location is directly above VM519. \\
\hline VT955 & Stack & Wall & $\begin{array}{l}\text { This location is south of VM520 at the same distance from the floor } \\
\text { level. The beta-gamma background was } 72 \mathrm{cpm}(0.04 \mathrm{mrad} / \mathrm{h}) \text { with } \\
\text { the probe facing up. }\end{array}$ \\
\hline VT956 & Stack & Wall & $\begin{array}{l}\text { VT957 was the sample taken for rad analysis near (directly east) this } \\
\text { location. }\end{array}$ \\
\hline VT959 & Drain 49 & Drain & $\begin{array}{l}\text { The beta-gamma was N.D. above background and sample VT958 } \\
\text { was taken at } 0 \text { to } 2 \text { in. depth for rad analysis. }\end{array}$ \\
\hline
\end{tabular}


Table 5. (continued)

\begin{tabular}{|c|c|c|c|}
\hline $\begin{array}{l}\text { Sample } \\
\text { ID } a\end{array}$ & $\begin{array}{l}\text { Room/ } \\
\text { structure/ L } \\
\text { area }^{b}\end{array}$ & $\begin{array}{l}\text { Location } \\
\text { in bay }\end{array}$ & Location notes $c$ \\
\hline VT960 & Drain 50 & Drain & $\begin{array}{l}\text { This } 5 \text { in. diameter drain (inside a } 1.5 \mathrm{ft} \times 1.5 \mathrm{ft} \text { pit) was cleaned by } \\
\text { KAPL before smear collection. Sample VT961 was taken at } 0 \text { to } 2.5 \\
\text { in. depth of this location for rad analysis. }\end{array}$ \\
\hline VT962 & $27 \mathrm{~W}$ & Wall & $\begin{array}{l}\text { The beta-gamma background in the coal room was } 40 \mathrm{cpm} 0.02 \\
\mathrm{mrad} / \mathrm{h} \text { ). Sample VT963 was taken just north of VT } 962 \text { for the rad } \\
\text { analysis sample. }\end{array}$ \\
\hline VT964 & $27 \mathrm{~W}$ & Bridging & The beta-gamma scan was N.D. above background at this location. \\
\hline VT965 & $27 \mathrm{~W}$ & Other & The beta-gamma scan was N.D. above background at this location. \\
\hline VT966 & $27 \mathrm{~W}$ & Bridging & Beta-gamma scan was N.D. above the background at this location. \\
\hline VT967 & $27 \mathrm{~W}$ & Stringer & Beta-gamma scan was N.D. above background at this location. \\
\hline VT968 & $27 \mathrm{~W}$ & Other & The beta-gamma scan was N.D. above background at this location. \\
\hline VT969 & $27 \mathrm{~W}$ & Bridging & The beta-gamma scan was N.D. above background at this location. \\
\hline VT970 & $27 \mathrm{~W}$ & Wall & The beta-gamma scan was N.D. above background at this location. \\
\hline VT971 & $27 \mathrm{~W}$ & Wall & $\begin{array}{l}\text { This location is } 4.5 \mathrm{ft} \text { above the boiler room floor level. The beta- } \\
\text { gamma scan was N.D. above background at this location and VT } 972 \\
\text { was taken as the rad sample directly south of VY971. }\end{array}$ \\
\hline VT973 & $27 \mathrm{~W}$ & Wall & $\begin{array}{l}\text { This location is } 10 \mathrm{ft} \text { above the boiler room floor level. The beta- } \\
\text { gamma scan was N.D. above background at this location. }\end{array}$ \\
\hline VT974 & $27 \mathrm{~W}$ & Wall & $\begin{array}{l}\text { This location is } 8 \mathrm{ft} \text { above the boiler room floor level. The beta- } \\
\text { gamma scan was N.D. above background at this location. }\end{array}$ \\
\hline VT975 & $27 \mathrm{~W}$ & Floor & Beta-gamma scan was N.D. above background \\
\hline VT976 & $27 \mathrm{~W}$ & Floor & The beta-gamma scan was N.D. above background. \\
\hline VT984 & $25 \mathrm{~W}$ & Drain & This is a repeat smear of sample VT898 (12-12-93). \\
\hline VT985 & $25 \mathrm{E}$ & Drain & This sample is a resmear of VT869 (12-11-93). \\
\hline VT986 & $5 \mathrm{~N}$ & Drain & This sample is a resmear of VT866 (12-11-93). \\
\hline VT987 & $26 \mathrm{~W}$ & Wall & $\begin{array}{l}\text { This boiler room overhead location was N.D. above background on } \\
\text { the beta-gamma scan. }\end{array}$ \\
\hline VT988 & $27 \mathrm{~W}$ & Other & The beta-gamma scan was N.D. above background. \\
\hline VT989 & $27 \mathrm{~W}$ & Bridging & The beta-gamma scan was N.D. above background. \\
\hline VT990 & $27 \mathrm{~W}$ & Other & $\begin{array}{l}\text { This was an overhead location in the boiler room. The beta-gamma } \\
\text { scan was N.D. above background. }\end{array}$ \\
\hline VT991 & $26 \mathrm{~W}$ & Other & The beta-gamma scan was N.D. above background. \\
\hline VT992 & $26 \mathrm{~W}$ & Bridging & The beta-gamma scan was N.D. above background. \\
\hline VT993 & $27 \mathrm{~W}$ & Floor & $\begin{array}{l}\text { The beta-gamma scan was N.D. above background. Sample VT994 } \\
\text { was taken nearby (just NW of VT993) for rad analysis. }\end{array}$ \\
\hline VT995 & $27 \mathrm{~W}$ & Floor & The beta-gamma scan was N.D. above background. \\
\hline
\end{tabular}


Table 5. (continued)

\begin{tabular}{|c|c|c|c|}
\hline $\begin{array}{c}\text { Sample } \\
\text { ID }{ }^{a}\end{array}$ & $\begin{array}{l}\text { Room/ } \\
\text { structure/ } \\
\text { areab }^{b}\end{array}$ & $\begin{array}{l}\text { Location } \\
\text { in bay }\end{array}$ & Location notesc \\
\hline VT996 & $26 \mathrm{~W}$ & Floor & The beta-gamma scan was N.D. above background. \\
\hline VT997 & $26 \mathrm{~W}$ & Floor & The beta-gamma scan was N.D. above background. \\
\hline VT998 & $26 \mathrm{~W}$ & Floor & $\begin{array}{l}\text { This location is between the boiler pits and "same height as above } \\
\text { mentioned floor area". The beta-gamma scan was N.D. above } \\
\text { background. }\end{array}$ \\
\hline VT999 & $26 \mathrm{~W}$ & Floor & $\begin{array}{l}\text { The beta-gamma scan was N.D. above background. Sample VT1000 } \\
\text { was taken nearby (just south of VT999) for rad analysis. }\end{array}$ \\
\hline VT1001 & $26 \mathrm{~W}$ & Window & The beta-gamma scan was N.D. above background. \\
\hline VT1002 & $27 \mathrm{~W}$ & Other & $\begin{array}{l}\text { This boiler room location had a beta-gamma scan of N.D. above } \\
\text { background. }\end{array}$ \\
\hline VT1003 & $25 \mathrm{~W}$ & Drain & $\begin{array}{l}\text { The beta-gamma scan was N.D. above background ( } 44 \mathrm{cpm}=0.02 \\
\mathrm{mrad} / \mathrm{h}) \text { at this location. }\end{array}$ \\
\hline VT1004 & $30 \mathrm{~W}$ & Wall & $\begin{array}{l}\text { This north tenant office space location is about } 4 \mathrm{ft} \text { above floor level } \\
\text { with the beta-gamma scan being N.D. above background }(44 \mathrm{cpm}= \\
0.02 \mathrm{mrad} / \mathrm{h} \text { ). }\end{array}$ \\
\hline VT1005 & $32 \mathrm{~W}$ & Wall & $\begin{array}{l}\text { This location is about } 8 \mathrm{ft} \text { above floor level and above the false } \\
\text { ceiling in the north tenant office space. The beta-gamma scan was } \\
\text { N.D. above background ( } 44 \mathrm{cpm}=0.02 \mathrm{mrad} / \mathrm{h} \text { ). }\end{array}$ \\
\hline
\end{tabular}

a Samples designated "VM" are dust/debris or insulation; "VT" samples are smears. Locations are shown on Figs. 3 through 7 . Not all numbers in the sample numbering system were used. This document and the document relating the details of the supplementary characterization survey [ORNL-6623 (Supplement)] considered together, contain all the data collected to assess the condition of the site.

bBeams are numbered; N, S, E. W., C designate north, south, eash, west, and center; $M S=$ metal shop; WS = wood shop.

$c$ N.D. $=$ none detectable $; B G=$ background. Counts per minute $(\mathrm{cpm})$ were converted to beta-gamma dose rates given in $\mathrm{mrad} / \mathrm{h}$. 


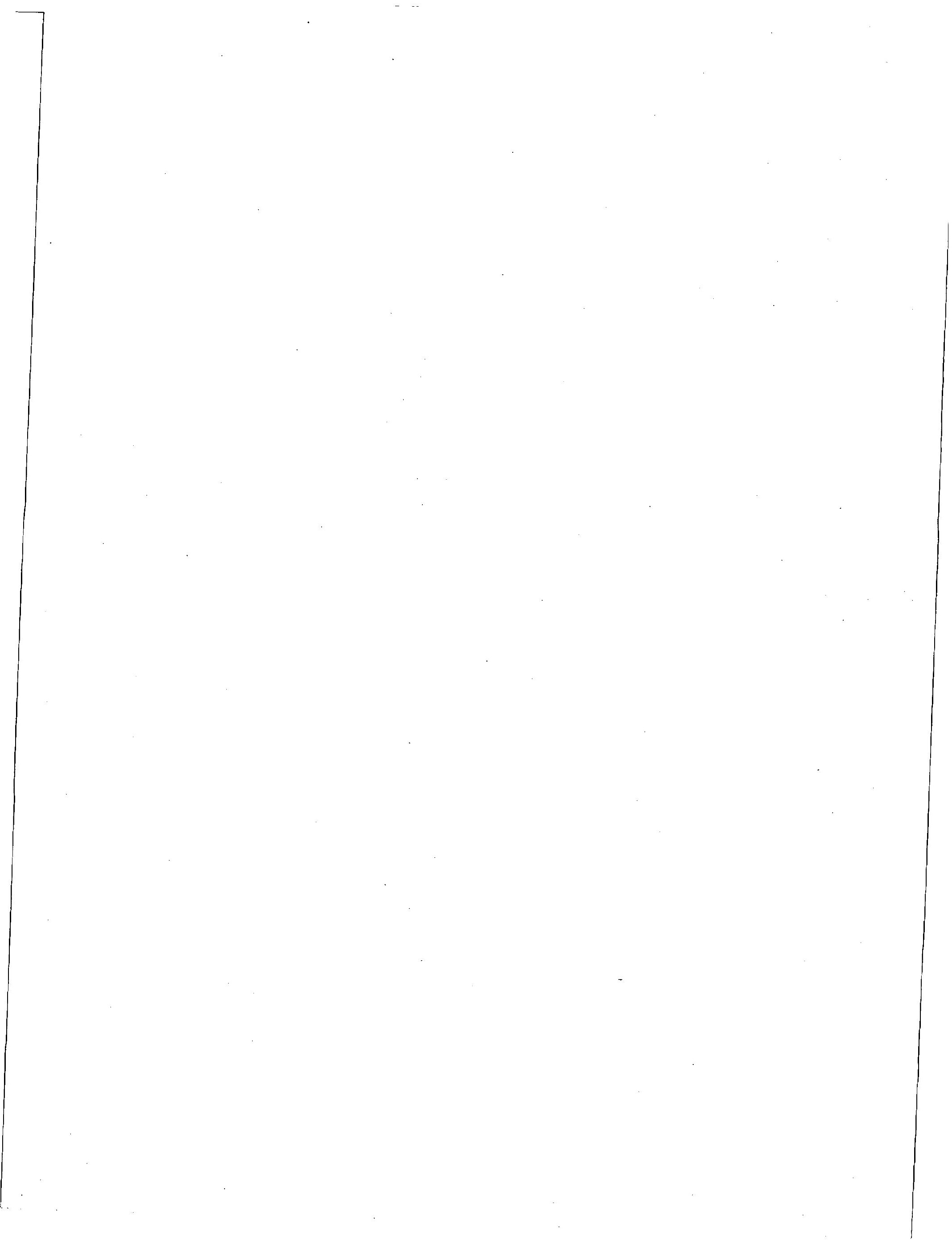


ORNL-6808

\section{INTERNAL DISTRIBUTION}

1. B. A. Berven

2. K. J. Brown

3. R. F. Carrier

4. W. D. Cottrell

5 -10. R. D. Foley

11-15. C. A. Johnson

16. M. E. Murray

17. P. T. Owen

18 D. A. Roberts
19. R. E. Rodriguez

20-22. R. E. Swaja

23. M. S. Uziel

24. J. K. Williams

25. Central Research Library

26-27. Laboratory Records

28. Laboratory Records-RC

29. ORNL Patent Section

30. ORNL Technical Library, Y-12

31-36. MAD Records Center

\section{EXTERNAL DISTRIBUTION}

37. James J. Fiore, Director, Office of Eastern Area Programs, Office of Environmental Restoration, U.S. Department of Energy, 4th Floor, 656 Quince Orchard Rd., Gaithersburg, MD 20878

38. L. K. Price, Director, Former Sites Restoration Division, Oak Ridge Field Office, U.S. Department of Energy, P.O. Box 2001, Oak Ridge, TN 37831-8723

39-139. A. R. Seepo, Department of Energy, Building M2-Warehouse, 2401 River Road, Schenectady, New York 12301-1069

140. James W. Wagoner II, Director, Division of Off-Site Programs, Office of Eastern Area Programs, Office of Environmental Restoration, U.S. Department of Energy, 4th Floor, 656 Quince Orchard Rd., Gaithersburg, MD 20878

141. A. Wallo III, Department of Energy, Environmental Guidance Division (EH-232) 1000 Independence Avenue, Washington DC 20585

142-143. W. A. Williams, Designation and Certification Manager, Division of Off-Site Programs, Office of Eastern Area Programs, Office of Environmental Restoration, U.S. Department of Energy, 4th Floor, 656 Quince Orchard Rd., Gaithersburg, MD 20878

144. C. D. Young, Office of Technical Services, Roy F. Weston, Inc., 12800 Middlebrook Road, Suite 207, Germantown; MD 20874

145-147. Office of Scientific and Technical Information, U.S. Department of Energy, P.O. Box 62, Oak Ridge, TN 37831

148. Office of Assistant Mgr. Energy Research and Development, U. S. DOE, P.O. Box 2008, Oak Ridge, TN, 37831-6269 